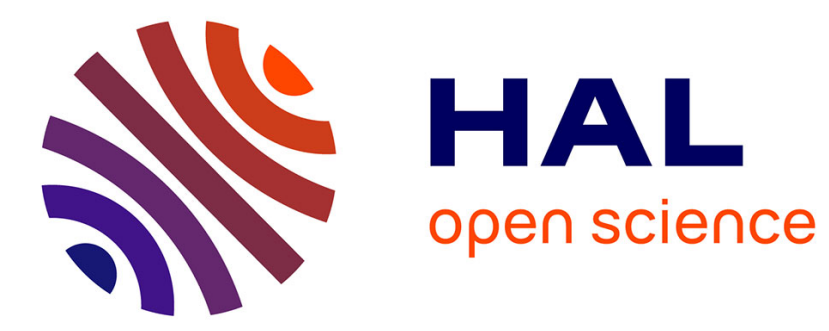

\title{
Shock wave refraction patterns at a slow-fast gas-gas interface at superknock relevant conditions
}

Yann de Gouvello, Mathilde Dutreuilh, Stany Gallier, J. Melguizo-Gavilanes, Rémy Mével

\section{- To cite this version:}

Yann de Gouvello, Mathilde Dutreuilh, Stany Gallier, J. Melguizo-Gavilanes, Rémy Mével. Shock wave refraction patterns at a slow-fast gas-gas interface at superknock relevant conditions. Physics of Fluids, 2021, 33 (11), pp.116101. 10.1063/5.0066345 hal-03424173

\section{HAL Id: hal-03424173 \\ https://hal.science/hal-03424173}

Submitted on 10 Nov 2021

HAL is a multi-disciplinary open access archive for the deposit and dissemination of scientific research documents, whether they are published or not. The documents may come from teaching and research institutions in France or abroad, or from public or private research centers.
L'archive ouverte pluridisciplinaire HAL, est destinée au dépôt et à la diffusion de documents scientifiques de niveau recherche, publiés ou non, émanant des établissements d'enseignement et de recherche français ou étrangers, des laboratoires publics ou privés. 


\section{Shock wave refraction patterns at a slow-fast gas-gas interface at super-knock relevant conditions}

Yann de Gouvello, ${ }^{1}$ Mathilde Dutreuilh, ${ }^{1}$ Stany Gallier, ${ }^{2}$ Josué Melguizo-Gavilanes, ${ }^{3,}$ a) and Rémy Mével (睿米) ${ }^{4}$, b)

1) ENSTA Paris, 91120 Palaiseau, France

${ }^{2)}$ ArianeGroup, Le Bouchet Research Center, 91710 Vert le Petit, France

3) Institut Pprime, UPR 3346 CNRS, ISAE-ENSMA, Université de Poitiers, 86961 Futuroscope-Chasseneuil, France

4) Center for Combustion Energy, School of Vehicle and Mobility, Tsinghua University, Beijing 100084, China

(Dated: 30 September 2021)

Shock wave refraction theory and high-resolution numerical simulations were employed to predict the refraction pattern under super-knock relevant conditions at slow-fast gas-gas interfaces which are characterized by a higher acoustic impedance in the incident phase than in the transmitted phase. First, our theoretical and computational methodologies were validated against results from the literature for planar shock-planar oblique interface interactions. Second, our framework was applied to planar shock-/cylindrical shock-cylindrical interface interactions. The theoretical regime diagram agrees well with the numerical predictions for the former configuration whereas significant discrepancies were observed for the latter. Numerical results show the formation of temperature and pressure peaks as the refraction structure transits from a Free Precursor Refraction to a Twin von Neumann Refraction. This change in thermodynamic state can induce a significant reduction in ignition delaytime, potentially leading to detonation onset.

Keywords: Shock wave refraction; Slow-fast interface; Regime diagram; Super-knock; Numerical simulation

\footnotetext{
a)Electronic mail: josue.melguizo-gavilanes@cnrs.pprime.fr

b)Electronic mail: mevel@mail.tsinghua.edu.cn
} 


\section{NOMENCLATURE}

\begin{tabular}{|c|c|c|c|}
\hline Acronyms & Definition & Symbols & \\
\hline AMR & Adaptive mesh refinement & Greek & Definition \\
\hline $\mathrm{BPR}$ & Bound precursor refraction & $\alpha$ & Angle between shock and interface \\
\hline CFL & Courant-Friedrichs-Lewy & $\chi$ & Inverse shock strength \\
\hline FNR & Free precursor von Neumann refraction & $\Delta$ & Grid size \\
\hline FPR & Free precursor refraction & $\delta$ & Flow deflection angle \\
\hline GFMP & Ghost fluid method for the poor & $\gamma$ & Ratio of specific heats \\
\hline HLLC & Harten-Lax-van Leer-Contact & $\omega_{i}$ & $\begin{array}{l}\text { Angle between the interface and the } \\
\text { trajectory of the end point } I_{p}\end{array}$ \\
\hline LSR & Lambda shock refraction & $\omega_{t}$ & $\begin{array}{l}\text { Angle between transmitted } \\
\text { shock and the interface }\end{array}$ \\
\hline MPI & Message passing interface & $\psi$ & Level set function \\
\hline $\mathrm{RCM}$ & Rapid compresion machine & $\rho$ & Gas density \\
\hline RRE & $\begin{array}{l}\text { Regular refraction with a reflected } \\
\text { expansion }\end{array}$ & $\tau_{\text {ind }}$ & Ignition delay-time \\
\hline RRR & $\begin{array}{l}\text { Regular refraction with a reflected } \\
\text { shock }\end{array}$ & $\xi$ & Shock strength \\
\hline SI-ICE & $\begin{array}{l}\text { Spark ignition internal combustion } \\
\text { engines }\end{array}$ & Subscript & Definition \\
\hline SWFID & $\begin{array}{l}\text { Shock wave and flame front induced } \\
\text { detonation }\end{array}$ & $p$ & Piston \\
\hline SWFoID & Shock wave focusing induced detonation & $i$ & Incident shock \\
\hline SWRID & Shock wave reflection induced detonation & $t$ & Transmitted shock \\
\hline TNR & Twin von Neumann refraction & $\omega$ & Shock created by piston compression \\
\hline TVD & Total variation diminishing & $n$ & normal component \\
\hline
\end{tabular}

WENO Weighted essentially non-oscillatory 


\begin{tabular}{|c|c|c|c|}
\hline \multicolumn{4}{|c|}{ Symbols } \\
\hline Latin & Definition & & \\
\hline$a$ & Speed of sound & $\mathrm{r}$ & Reflected wave \\
\hline$c$ & Discriminant & $\mathrm{sl}$ & Slip line \\
\hline$C_{v}$ & $\begin{array}{l}\text { Heat capacity at constant } \\
\text { volume }\end{array}$ & $T$ & Gas temperature \\
\hline E & Total energy & $t_{I_{p}}$ & Trajectory of point $I_{p}$ \\
\hline$e_{\text {int }}$ & Internal energy & $u$ & Horizontal velocity component \\
\hline$e_{\text {kin }}$ & Kinetic energy & $V$ & Velocity (scalar) \\
\hline $\boldsymbol{F}$ & $\begin{array}{l}\text { Convective flux vector } \\
x \text {-direction }\end{array}$ & $v$ & Vertical velocity component \\
\hline$G$ & $\begin{array}{l}\text { Convective flux vector } \\
y \text {-direction }\end{array}$ & $v$ & Gas specific volume \\
\hline$I_{p}$ & End point incident shock & $X$ & Mole fraction \\
\hline$j$ & $j$-shock & Numbers & \\
\hline$k$ & $k$-shock & $\mathbf{I}$ & $\begin{array}{l}\text { Phase with high acoustic } \\
\text { impedance }\end{array}$ \\
\hline$M$ & Mach number & II & $\begin{array}{l}\text { Phase with low acoustic } \\
\text { impedance }\end{array}$ \\
\hline $\mathcal{M}$ & Molar mass & 0 & State ahead of the incident shock \\
\hline $\mathrm{m}$ & Gas-gas interface & 1 & State behind the incident shock \\
\hline$n$ & $n$-shock & 3 & State behind of the reflected shock \\
\hline$p$ & Gas pressure & 4 & State behind of the transmitted shock \\
\hline$Q$ & $\begin{array}{l}\text { Vector of conserved } \\
\text { variables }\end{array}$ & 5 & State ahead of the transmitted shock \\
\hline$R$ & Radius of cylindrical interfa & & \\
\hline
\end{tabular}




\section{INTRODUCTION}

One of the most promising strategies to improve the efficiency of spark ignition internal combustion engines (SI-ICE) is to increase the power density through high boosting of downsized engines ${ }^{1-3}$. In practice, this is achieved by a compression ratio increase in the engine's cylinder ${ }^{4}$. However, under such conditions, super-knock is likely to occur ${ }^{1}$. Super-knock corresponds to the initiation of a detonation in the engine's cylinder and is a major obstacle for the development of next generation SI-ICE. Super-knock events are characterized by high-frequency/large-amplitude pressure oscillations, i.e., several MPa. Due to these extreme pressure peaks and oscillation amplitudes, a single super-knock event can result in irreversible damage to the engine. Super-knock is typically observed in the low-speed, high-load regime and includes three phases: (i) pre-ignition; (ii) auto-ignition in the end-gas; and (iii) detonation initiation and propagation. Pre-ignition is a random event and is related to the formation of a hot spot from a lubricating oil droplet or from a carbon deposit ${ }^{3}$. Auto-ignition in the end-gas is due to the high temperature and pressure attained in this region. A local explosion in the compressed fresh mixture is observed and the rapid expansion of the burning gas induces the formation of a shock wave ${ }^{3,5-7}$. Detonation initiation, or super-knock onset, results from the shock wave initiated by the end-gas local explosion upon interaction with the engine's confinement, the pre-ignition flame, or another shock wave created at a different location within the end-gas ${ }^{3,5-7}$.

Experiments performed in a rapid compression machine (RCM) have enabled the identification of three modes, or mechanisms, of super-knock initiation based on the dynamics of the shock wave generated by the local explosion in the end-gas. The energy density of the reactive mixture was found to be the dominant parameter determining which mode of super-knock onset is taking place ${ }^{2,6,7}$. The first mode is referred to as Shock Wave Reflection Induced Detonation (SWRID). It is characterized by the formation of a Mach reflection as the shock wave formed by the local end-gas explosion propagates along the RCM wall. The high temperature and pressure behind the Mach stem initiates a detonation that propagates within the compressed end-gas and generates pressure oscillations ${ }^{6}$. The second mode is referred to as Shock Wave and Flame front Induced Detonation (SWFID). It is characterized by the formation of a shock wave refraction which creates a region of high pressure 
and temperature which enables flame front acceleration and subsequent detonation onset ${ }^{7}$. The third mode is referred to as Shock Wave Focusing Induced Detonation (SWFoID). Under certain circumstances, two (or more) local explosions in the end-gas can take place simultaneously or with a slight offset. The shock waves induced by these explosions can subsequently interact with each other, creating regions of high temperature and pressure by shock focusing; detonation initiation is thus likely to occur as a result of these interactions.

The present paper focuses on the second mode of super-knock initiation during which shock wave refraction takes place and results in detonation onset. Wang et al. ${ }^{7}$ showed by combining high-speed experimental imaging and numerical simulation that the interplay between the shock wave generated by the end-gas auto-ignition and the spherical flame results in thermodynamic conditions that are prone to flame acceleration and detonation onset. However, the high computational cost of the reactive simulation performed by Wang et al. ${ }^{7}$ prevented the use of adequate resolution to resolve the structure of the refraction pattern. Shock wave refraction is a complex interaction between a shock wave and an interface (gas-gas or gas-liquid) ${ }^{8}$. In the context of super-knock, only the slow-fast gas-gas interfaces are of interest, where slow/fast refer to a low/high sound speed, and are thus characterized by a higher acoustic impedance in the incident phase than in the transmitted phase. Planar shock wave refraction at non-reactive slow-fast gas-gas interface has been extensively studied experimentally, numerically, and theoretically by Henderson's group ${ }^{10-14}$ and a theoretical regime diagram was established ${ }^{11}$. Several other studies have been performed on planar shock wave refraction over linear, square, triangular, cylindrical, spherical, elliptic, V-shaped, non-reactive gas-gas interfaces ${ }^{15-24}$. Refraction in non-reactive mixtures were also studied for converging cylindrical shock interacting with a straight interface ${ }^{25}$. Concerning reactive mixtures, two situations can be distinguished: (i) ignition during planar shock wave refraction at an interface between two cold gases, and (ii) ignition after planar shock refraction has taken place at an interface between a cold gas and a flame. The first situation has been studied experimentally by Haehn et al. ${ }^{26}$ and numerically by Diegelmann et al. ${ }^{27-29}$, but for a fast-slow interface. The second situation has been studied experimentally for example by Thomas et al. ${ }^{30}$, and numerically by Bakalis et al. ${ }^{31}$. Under such a configuration, ignition, and possibly detonation onset, is attributed to the acceleration of the flame by the turbulence induced by Richtmyer-Meshkov instabilities. Note that 
Bakalis et al. ${ }^{31}$ indicated that "a single weak shock-flame interaction is insufficient to cause a prompt DDT" which is in contradiction with the SWFID mechanism. From our literature review, it seems that only planar shock waves have been considered previously and that studies on refraction in reactive mixtures are scarce. A better understanding of super-knock initiation by shock wave refraction thus requires a detailed knowledge of the structure and dynamics of the refracting shock wave. The goals of the present study are (i) to assess the capabilities of the existing theory in predicting the refraction structure under super-knock relevant conditions, and (ii) to identify through high-resolution numerical simulations which refraction pattern is the most likely to favor ignition.

The manuscript is organized as follows: section II provides a detailed description of the possible regular and irregular refraction structures; section III and IV present the computational and theoretical methodologies; section $\mathrm{V}$ provides a thorough description of the framework to compute a theoretical shock refraction regime diagram at a slow-fast interface, and validation with previous experimental results and simulations; section VI includes results and discussion for shock wave refraction patterns at super-knock relevant conditions; concluding remarks are included in section VII.

\section{REGULAR AND IRREGULAR REFRACTION PATTERNS}

Using shock polars, a shock refraction regime diagram like the one shown in Figure 1 can be constructed in the $\omega_{i-} \chi$ space, where $\omega_{i}$ is the angle between the interface and the trajectory of the end point of the incident shock, and $\chi=p_{0} / p_{1}$ is the inverse shock strength, where subscripts 0 and 1 denote the pressure ahead and behind the incident shock. For a fixed $\chi$ and increasing $\omega_{i}$ one can traverse all the possible refraction patterns and identify their transition limits. The shock wave patterns observed at a slow-fast interface are divided into regular and irregular refraction patterns. For each pattern, a polar diagram can be drawn, similar to those routinely used to study shock reflections ${ }^{32}$. Next, we provide a detailed description of the patterns observed using schematics. 


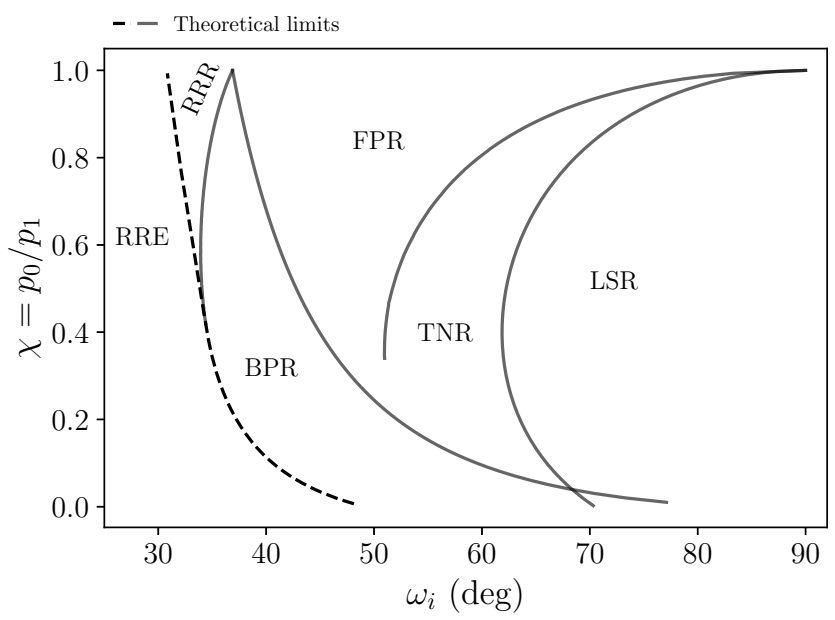

FIG. 1. Theoretical regime diagram for shock refraction patterns at a slow-fast interface. $\chi=$ $p_{0} / p_{1}$ is the inverse pressure ratio, and $\omega_{i}$ is angle between the interface and the the trajectory of the end point of the incident shock. RRE: Regular Refraction with reflected Expansion. RRR: Regular Refraction with reflected Shock. BPR: Bound Precursor Refraction. FPR: Free Precursor Refraction. TNR: Twin von Neumann Refraction. LSR: Lambda Shock Refraction.

\section{A. Regular refraction patterns}

Regular refraction patterns correspond to patterns for which the incident, transmitted and reflected waves meet at a point located on the gas-gas interface (i.e., the triple point $)^{10}$. The possible regular refraction patterns are shown in Figure 2. Three patterns can be distinguished: (i) the Regular Refraction with a reflected Expansion (RRE) - see Figure 2 a); (ii) the transition from reflected expansion to reflected shock - see Figure 2 b); and (iii) the Regular Refraction with a Reflected shock (RRR) - see Figure 2 c). In Figure 2, the angle $\alpha$ corresponds to the angle between the incident shock and the interface. The role of the reflected wave is to equalize the pressure on either side of the disturbed interface and to deflect the flows so that they are parallel to each other and to the disturbed interface. Depending on the pressure difference behind the incident and the transmitted wave, either an expansion wave or a compression wave is required to satisfy $p_{3}=p_{4}$, see Figure 2 a). At 
the transition, the pressure difference behind the incident and the transmitted wave is equal to zero, so that the reflected wave is a Mach wave (i.e., a zero-strength wave whose pressure ratio is unity). At slow-fast interfaces, regular refraction structures usually occur at small angles of incidence, $\omega_{i}$, although the strength of the shock can influence the nature of the reflected shock ${ }^{10,11,33}$. See RRR and RRE limits in Figure 1.

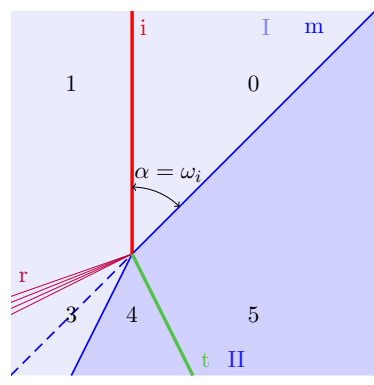

(a) $\mathrm{RRE}$

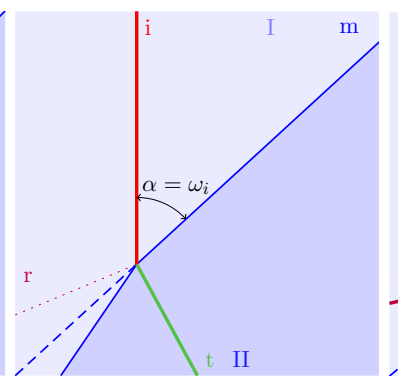

(b) Transition RRE $\rightarrow$ RRR

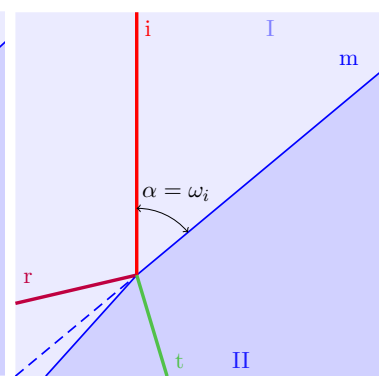

(c) RRR

FIG. 2. Schematic of the possible regular refraction patterns: (a) Regular Refraction with reflected Expansion (RRE); (b) regular transition pattern (RRE $\rightarrow$ RRR); and (c) Regular Refraction with Reflected shock (RRR). Propagation from left to right. i: incident wave; t: transmitted wave; r: reflected wave; m: interface; I and II: phases I and II. 0: initial state in phase I. 5: initial state in phase II. 1: state behind the incident shock in phase I. 3: state behind the reflected wave in phase I. 4: state behind the transmitted shock in phase II. The dashed blue line is a visual aid showing the initial position of the interface before interaction with the incident shock wave (i).

\section{B. Irregular refraction patterns}

With increasing angle of incidence, the refraction pattern becomes irregular. Irregular refraction patterns correspond to patterns for which the transmitted shock wave moves ahead of the incident wave and is referred to as "precursor wave" in literature ${ }^{10}$; a triple point no longer exists on the interface in this type of interactions. The possible irregular refraction patterns are shown in Figure 3. Five patterns can be distinguished, from Figure 3 a) to e) these are: (i) the Bound Precursor Refraction (BPR); (ii) the Free precursor von Neumann Refraction (FNR); (iii) the Free Precursor Refraction (FPR); (iv) the Twin von 
Neumann Refraction (TNR); and (v) the Lambda Shock Refraction (LSR). The BPR pattern corresponds to the transition between regular and irregular refraction. The structure of the BPR is close to that of a RRR but with a curved transmitted wave that propagates ahead of the incident wave but at nearly the same speed ${ }^{9}$. For free precursor patterns, i.e., FNR, FPR, TNR, LSR, the transmitted shock is faster than the incident shock and new shocks form, labeled $j, k$, and $n$ in Figure $3 \mathrm{~b}$ )-e), connecting the transmitted wave to the incident and reflected shocks. The structures of the free precursor refraction are much more complex than those of regular patterns. The FNR and FPR patterns are quite similar except that, in the FNR case, the $k$-shock undergoes a Mach reflection at the interface whereas in the FPR case it remains undisturbed ${ }^{9}$. At larger angles of incidence, $\omega_{i}$, the interaction of $j$-shock and the incident wave leads to the formation of the TNR pattern which is characterized by two triple points and two slip lines ${ }^{9}$. A further increase in $\omega_{i}$, results in the reflected shock becoming a Mach wave as the flow behind the incident shock approaches sonic conditions and the LSR structure is formed ${ }^{9}$. 


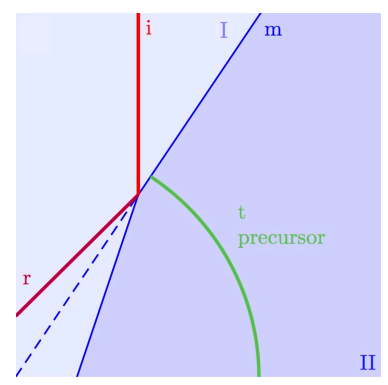

(a) BPR

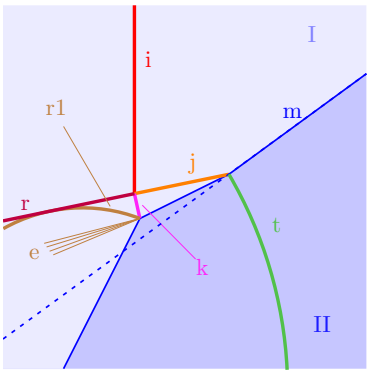

(c) FPR

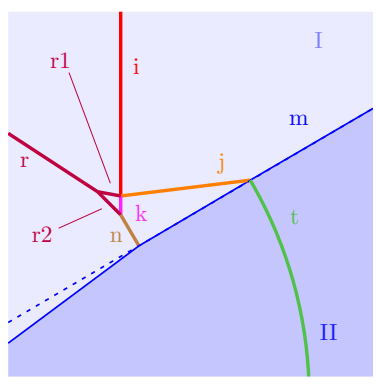

(b) FNR

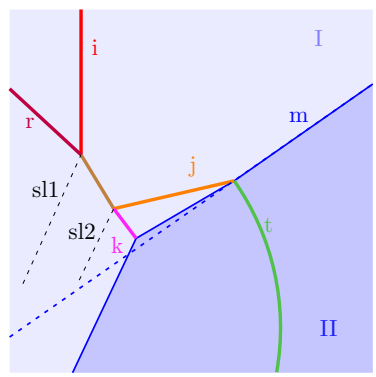

(d) TNR

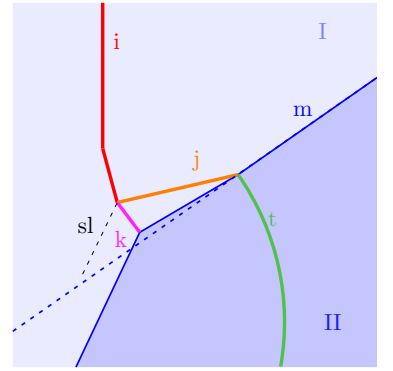

(e) LSR

FIG. 3. Schematic of the possible irregular refraction patterns: (a) Bound Precursor Refraction (BPR); (b) Free von Neumann Refraction (FNR); (c) Free Precursor Refraction (FPR); (d) Twin von Neumann Refraction (TNR); (e) and Lambda Shock Refraction (LSR). Propagation from left to right. i: incident wave; t: transmitted wave; $\mathrm{r}$ : reflected wave; m: interface; $\mathrm{j}: j$-shock; $\mathrm{k}$ : $k$-shock; n: $n$-shock; and sl: slip line. The dashed blue line is a visual aid showing the initial position of the interface before interaction with the incident shock wave (i). Note that potential interface roll-up is not depicted, and the angles $\alpha$ and $\omega_{i}$ are not displayed for clarity. 


\section{COMPUTATIONAL METHODOLOGY}

\section{A. Governing equations}

We are primarily interested in the shock structures that form at the interface between two gases. Due to the high Reynolds numbers expected, viscous effects are neglected, and the problem is governed by the Euler equations. To enable a meaningful comparison between simulations and theoretical predictions, each gas is assumed as inert and to have constant thermodynamic properties. Conservation of mass, momentum, and energy read:

$$
\frac{\partial \mathbf{Q}}{\partial t}+\frac{\partial \mathbf{F}}{\partial x}+\frac{\partial \mathbf{G}}{\partial y}=0
$$

where $\mathbf{Q}$ is the vector of conserved variables, and $\mathbf{F}$ and $\mathbf{G}$ are the convective fluxes in $x-$ and $y$-direction, respectively

$$
\mathbf{Q}=\left(\begin{array}{c}
\rho \\
\rho u \\
\rho v \\
\rho E
\end{array}\right) \quad \mathbf{F}=\left(\begin{array}{c}
\rho u \\
\rho u^{2}+p \\
\rho u v \\
\rho E u+p u
\end{array}\right) \quad \mathbf{G}=\left(\begin{array}{c}
\rho v \\
\rho u v \\
\rho v^{2}+p \\
\rho E v+p v
\end{array}\right)
$$

in $\mathbf{Q}, \mathbf{F}$ and $\mathbf{G}, \rho$ is the density, $p$ is the pressure, $(u, v)$ are the horizontal and vertical velocity components, and $E$ the total energy which is the sum of the internal energy and kinetic energy, $E=e_{\text {int }}+e_{\text {kin }}$ with $e_{\text {kin }}=\left(u^{2}+v^{2}\right) / 2$. Both gases are calorically perfect: $e_{\text {int }}=p /(\gamma-1) \rho$ with $\gamma$ denoting the ratio of specific heats.

Special attention is paid to the interface between the two gases. It is captured using a level-set approach avoiding, to the extent possible, numerical diffusion and defined by the zero-level of the level-set function $\psi$ (i.e., $\psi=0$ ). Because the interface is advected at the local flow velocity, an additional transport equation needs being solved:

$$
\frac{\partial \psi}{\partial t}+u \frac{\partial \psi}{\partial x}+v \frac{\partial \psi}{\partial y}=0
$$

Equation (3) is recast in conservation form and solved for $\rho \psi$ with $x-$ and $y$-fluxes given by $\rho u \psi$ and $\rho v \psi$, and integrated along with system (1). Classically, $\psi$ is chosen as a signeddistance function to the interface that is prescribed at the beginning of the computation. However, since the distance property $(|\nabla \psi|=1)$ cannot be enforced in our particular case, we reinitialize the level-set at every iteration using the constrained re-initialization technique to avoid any order of accuracy and robustness issues ${ }^{34}$. 


\section{B. Numerical techniques}

The system of equations (1) was discretized using a finite volume technique and solved in a fixed Cartesian grid. Convective fluxes on cell edges are estimated using a HLLC Riemann solver $^{35}$, and primitive variables reconstructed at the interface using a fifth-order WENO scheme $^{36}$. Time integration is performed using a Total Variation Diminishing (TVD) thirdorder explicit Runge-Kutta method ${ }^{37}$. All the computations below were run using a CFL number of 0.6 .

Numerical treatment of the Euler equations at the interface between fluids having dissimilar properties or equations of state requires special care. It is well known that numerical solutions of such multi-medium flows are likely to show spurious oscillations in pressure and temperature at the interface. To effectively suppress them, we use the Ghost Fluid Method for the Poor (GFMP) technique ${ }^{38}$ where two numerical fluxes are computed at the material interface using the corresponding thermodynamic parameters of the fluids on either side of it. Note that while these two fluxes are identical for a cell interface separating two cell centers in the same material, they will differ across a material discontinuity. The resulting scheme is therefore locally non-conservative but conservation errors are known to decrease as the resolution increases ${ }^{38}$.

\section{Domain, initial and boundary conditions}

Two configurations were examined in the present study: (i) planar shock-oblique interface interactions, and (ii) planar/cylindrical shock-curved interface interactions (i.e., a bubblelike interface).

\section{Planar oblique interfaces}

To enable a direct comparison with the theoretical predictions, we considered a planar oblique interface between carbon dioxide $\left(\mathrm{CO}_{2}\right)$ and methane $\left(\mathrm{CH}_{4}\right)$ as this is a simple case for validation purposes. The configuration is sketched in Fig. 4. The interface is defined as an oblique line at an angle $90^{\circ}-\alpha$, located $10 \mathrm{~mm}$ from the left boundary. The numerical domain is a square region of size $L_{x}=L_{y}=20 \mathrm{~mm}$ filled with gas at rest, $\left(u_{0}, v_{0}\right)=0$, at ambient pressure and temperature $\left(p_{0}=0.1 \mathrm{MPa}\right.$ and $\left.T_{0}=300 \mathrm{~K}\right)$. These conditions 
correspond to those used by Abd-El-Fattah and Henderson ${ }^{11}$ in their experiments.

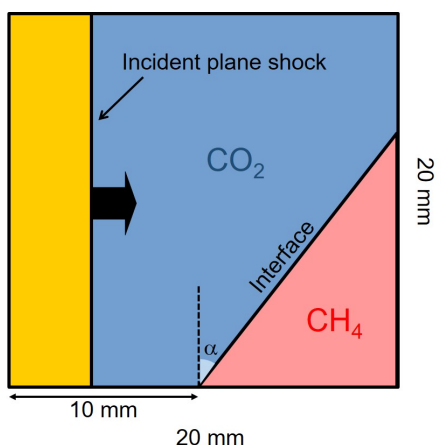

FIG. 4. Schematic of the numerical domain for planar shock-planar oblique interface interaction.

The post-shock conditions for the planar incident shock wave (initially located $5 \mathrm{~mm}$ from the left boundary) were obtained using normal shock relationships for two incoming Mach numbers, $M$, and subsequently changing the frame of reference to the laboratory frame. Table III C 1 lists the post-shock conditions denoted by subscript 1; the inverse incident shock strength, $\chi=p_{0} / p_{1}$, is also shown.

TABLE I. Post-shock conditions for planar shock-planar oblique interface interaction $\left(\mathrm{CO}_{2}-\mathrm{CH}_{4}\right)$.

\begin{tabular}{ccccc}
\hline$M$ & $\chi=p_{0} / p_{1}$ & $p_{1}(\mathrm{MPa})$ & $u_{1}(\mathrm{~m} / \mathrm{s})$ & $T_{1}(\mathrm{~K})$ \\
\hline 1.12 & 0.767 & 0.1303 & 53.7 & 313 \\
1.37 & 0.494 & 0.2026 & 152.7 & 353 \\
\hline \hline
\end{tabular}

As mentioned above, thermodynamic properties were assumed as constant for each gas. The ratio of specific heats, $\gamma$, the heat capacity at constant volume, $C_{v}$, and molar mass, $\mathcal{M}$ are $\gamma_{\mathrm{CO}_{2}}=1.288, C_{v, \mathrm{CO}_{2}}=656 \mathrm{~J} / \mathrm{kg} \mathrm{K}, \mathcal{M}_{\mathrm{CO}_{2}}=44.1 \mathrm{~g} / \mathrm{mol}, \gamma_{\mathrm{CH}_{4}}=1.303, C_{v, \mathrm{CH}_{4}}=$ $1711 \mathrm{~J} / \mathrm{kg} \mathrm{K}, \mathcal{M}_{\mathrm{CH}_{4}}=16.04 \mathrm{~g} / \mathrm{mol}$. The ratio of sound speeds and acoustic impedances for the $\mathrm{CO}_{2} / \mathrm{CH}_{4}$ interface considered are 0.6 (slow-fast) and 1.65 , respectively. 


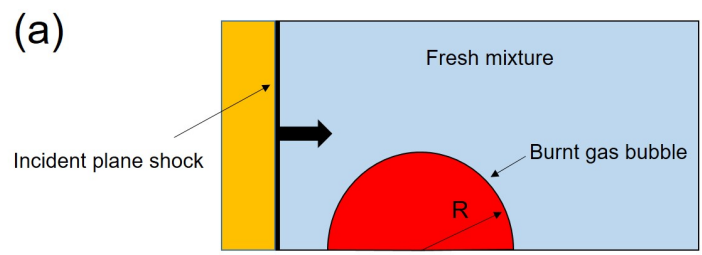

(b)

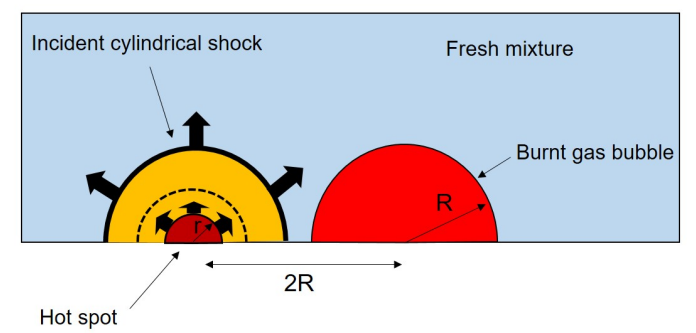

FIG. 5. Schematic for curved interface interactions with (a) a planar shock, and (b) a cylindrical shock. $R=12.5 \mathrm{~mm}$ and $r_{0}=3.75 \mathrm{~mm}$.

\section{Curved interfaces}

To address a situation that is more relevant to super-knock conditions in SI-ICE, we consider a hemi-cylindrical bubble of radius $R=12.5 \mathrm{~mm}$ filled with hot, burnt gas placed in a fresh iso-octane $\left(\mathrm{C}_{8} \mathrm{H}_{18}\right)$-air mixture and make it interact with planar or cylindrical incident shocks; see Fig. 5 a) and b). The main motivation to study this configuration is to evaluate the role of the shock and interface curvature on the structure of the refraction pattern. For the planar case the bubble center is located $20 \mathrm{~mm}$ away from the left boundary, whereas the hemi-cylindrical shock was generated from a high pressure/temperature region (i.e., a hot spot) of initial radius $r_{0}=3.75 \mathrm{~mm}$ ) located $2 R=25 \mathrm{~mm}$ away from the burnt gas bubble. The above mentioned structures are specified within a rectangular domain of size $L_{x}=40 \mathrm{~mm} \times L_{y}=25 \mathrm{~mm}$ and $L_{x}=53 \mathrm{~mm} \times L_{y}=30 \mathrm{~mm}$ for planar- and curved-shocks, respectively.

Initial conditions and compositions of the fresh and burnt mixtures are listed in Tab. III C 2. The conditions in the burnt mixture were computed assuming that (i) the burnt mixture is in chemical equilibrium at constant pressure, (ii) complete conversion of the reactants to 
water vapor and carbon dioxide is achieved, and (iii) nitrogen is an inert chemical species. The table also shows the thermodynamic properties $\gamma$ and $C_{v}$.

TABLE II. Initial conditions for planar/curved shock-curved interface interactions.

\begin{tabular}{lcc}
\hline \hline & Fresh mixture Burnt mixture \\
\hline$p_{0}(\mathrm{MPa})$ & 7.5 & 7.5 \\
$T_{0}(\mathrm{~K})$ & 820 & 2500 \\
$X_{\mathrm{CO}_{2}}$ & - & 0.12500 \\
$X_{\mathrm{H}_{2} \mathrm{O}}$ & - & 0.14063 \\
$X_{\mathrm{N}_{2}}$ & 0.77686 & 0.73437 \\
$X_{\mathrm{O}_{2}}$ & 0.20661 & - \\
$X_{\mathrm{C}_{8} \mathrm{H}_{18}}$ & 0.01653 & - \\
$\gamma$ & 1.279 & 1.246 \\
$C_{v}(\mathrm{~J} / \mathrm{kgK})$ & 985 & 1185 \\
\hline \hline
\end{tabular}

Three incident shock Mach numbers were considered. The post-shock fresh mixture properties are presented in Tab III. For the planar shock configuration, the same methodology as that described in section III C 1 was used. For the hemi-cylindrical shock case, the initial pressure and density inside the hot spot were adjusted to obtain comparable conditions as in the planar case, at the time when the cylindrical shock meets the symmetry axis $(y=0)$ of the bubble filled with burnt gas. This allows for a more meaningful comparison between the planar and cylindrical shock cases.

TABLE III. Post-shock conditions for planar/curved shock-curved interface interactions.

\begin{tabular}{|c|c|c|c|c|}
\hline \multicolumn{5}{|c|}{$M \chi=p_{0} / p_{1} p_{1}(\mathrm{MPa}) u_{1}(\mathrm{~m} / \mathrm{s}) T_{1}(\mathrm{~K})$} \\
\hline 1.1 & 0.809 & 9.27 & 90 & 859 \\
\hline 1.5 & 0.416 & 18.03 & 393 & 1010 \\
\hline 2.0 & 0.228 & 32.90 & 709 & 1226 \\
\hline
\end{tabular}

The domain, initial and boundary conditions chosen correspond to those used in the super-knock experiments performed by Wang et al..$^{5-7}$. Further discussion is provided in 
section VIA.

\section{Mesh and boundary conditions}

All simulations presented use a spatial resolution of $\Delta=10 \mu \mathrm{m}$, which is the same as in the shock wave refraction study performed by Nourgaliev et al. ${ }^{8}$. Halving or doubling the resolution did not result in appreciable changes on the refraction patterns obtained close to boundaries; a sample result in shown is Figure 6 for a nascent TNR structure. The total number of cells within the numerical domains were $10 \times 10^{6}$ and $15.9 \times 10^{6}$ for planar and curved shock-interface interactions. As for boundary conditions, on the left, a supersonic inlet and zeroth order extrapolation were used for planar shocks and curved shocks, respectively. On the bottom, symmetry for all cases, and the right/top boundaries are updated by extrapolating from the interior solution assuming continuity at the boundary. Our code is fully parallelized (MPI) and shows linear scaling up to 1,000 cores. The cost per simulation was $3072 \mathrm{CPU}$ hours (i.e., 6 hours on 512 cores).
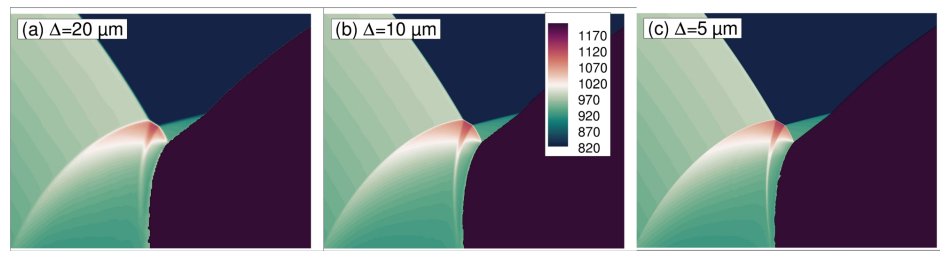

FIG. 6. Effect of grid resolution $\Delta$ (20 $\mu \mathrm{m}$ (a); $10 \mu \mathrm{m}$ (b) ; $5 \mu \mathrm{m}$ (c)) on the temperature field of a nascent TNR structure. Simulation results obtained for a cylindrical interface and a cylindrical shock wave propagating at $M=1.5 \alpha=74.7^{\circ} ; \omega_{i}=71.2^{\circ}$.

\section{THEORETICAL METHODOLOGY}

Abd-El-Fattah and Henderson ${ }^{11}$ developed a methodology to determine the regime diagram of shock refraction at slow-fast interface as a function of the inverse incident shock strength, $\chi=p_{0} / p_{1}$, and a reference angle, $\omega_{i}$, defined as the angle between the incident shock and the trajectory of the end point of the incident shock, denoted $I_{p}$ in Figure 7. 
For regular refraction cases $(\mathrm{RRR} / \mathrm{RRE})$ and $\mathrm{BPR}, I_{p}$ is always located on the interface, whereas for irregular refraction cases, $I_{p}$ is located away from the interface. As mentioned above, $\alpha$ is the angle between the interface and the incident shock. For regular refractions, $\alpha=\omega_{i}$, whereas for irregular refractions, $\alpha \neq \omega_{i}$. Angles $\alpha$ and $\omega_{i}$ are also shown in Figure 7 for both regular and irregular refraction patterns. For the case of a curved incident shock, angles are measured relative to the tangent line of the incident shock at $I_{p}$. Before proceeding with details of the method to determine the refraction regime diagram, we recall, for completeness, the oblique shock equations as well as the piston theory.

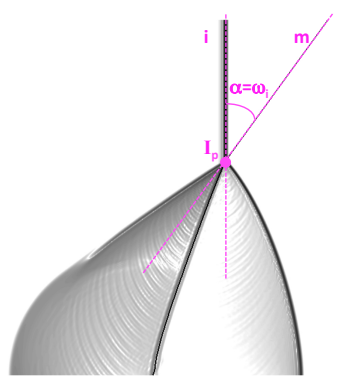

(a) BPR, $\alpha=\omega_{i}=35^{\circ}$

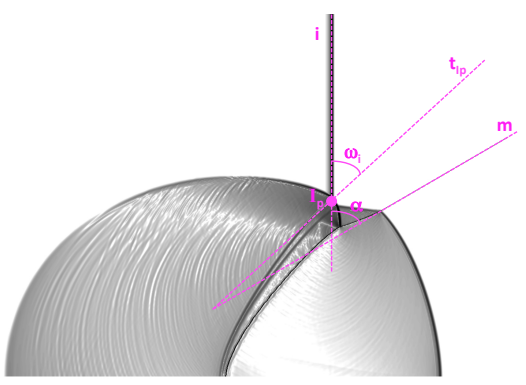

(b) TNR, $\alpha=60^{\circ}, \omega_{i}=52.9^{\circ}$

FIG. 7. Definition of $\alpha$ and $\omega_{i}$ for (a) BPR and (b) TNR patterns at a slow-fast $\mathrm{CO}_{2}-\mathrm{CH}_{4}$ interface. Conditions: $\gamma_{\mathrm{CO}_{2}}=1.288 ; \gamma_{\mathrm{CH}_{4}}=1.303 ; \mathcal{M}_{\mathrm{CO}_{2}}=44.01 \mathrm{~g} / \mathrm{mol} ; \mathcal{M}_{\mathrm{CH}_{4}}=16.04 \mathrm{~g} / \mathrm{mol}$; $M=1.37(\chi=0.494) . \mathrm{t}_{I_{p}}$ corresponds to the trajectory of the $I_{p}$ point.

\section{A. Oblique shock equations}

In Figure 8 a schematic for a typical RRE refraction structure in which the pre-/postshock states, flow directions and corresponding deflections, $\delta$, as the flow crosses over the incident, transmitted shocks and the reflected expansion are shown. For a calorically perfect gas (i.e., constant $\gamma$ ) the pressure, specific volume $(v=1 / \rho)$, and temperature ratios across an oblique shock wave are given by

$$
\xi=1 / \chi=\frac{p_{1}}{p_{0}}=\frac{1-\gamma+2 \gamma M_{0, n}^{2}}{\gamma+1} ; \quad \frac{v_{1}}{v_{0}}=\frac{(\gamma+1) M_{0, n}^{2}}{2+(\gamma-1) M_{0, n}^{2}} ; \quad \frac{T_{1}}{T_{0}}=\frac{p_{1}}{p_{0}} \frac{v_{1}}{v_{0}} .
$$

In Equation $4, M_{0, n}$ is the flow Mach number normal to the oblique incident shock wave in a frame of reference attached to the wave. It is related to the flow Mach number ahead, $M_{0}$, 


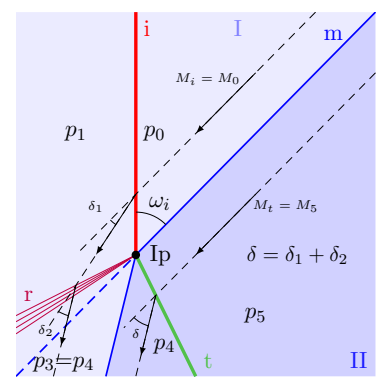

FIG. 8. Sample schematic showing the interaction of a planar shock wave with an oblique interface (RRE case). Note that the frame of reference is rotated and aligned with the interface; the arrows indicate the flow direction and corresponding deflections, $\delta$, as the flow crosses over the incident and transmitted shocks as well as over the reflected expansion.

through

$$
M_{0, n}=M_{0} \sin \left(\omega_{i}\right),
$$

The normal flow Mach number behind the oblique shock $\left(M_{1, n}\right)$ is

$$
M_{1, n}^{2}=\frac{1+M_{0, n}^{2}(\gamma-1) / 2}{\gamma M_{0, n}^{2}-(\gamma-1) / 2}
$$

and can be related to $M_{1}$ by

$$
M_{1, n}=M_{1} \sin \left(\omega_{i}-\delta\right),
$$

where $\delta$ is the flow deflection angle across the oblique shock and is related to $\omega_{i}$ by

$$
\tan (\delta)=2 \cot \left(\omega_{i}\right) \frac{M_{0, n}^{2}-1}{M_{0, n}^{2}\left(\gamma+\cos \left(2 \omega_{i}\right)\right)+2} .
$$

\section{B. Piston theory}

The velocity of a piston moving in a shock tube, $V_{p}$, and the velocity of the shock created by the piston compressing the gas, $V_{w}$, can be related by

$$
V_{p}=\frac{2}{\gamma+1} \frac{V_{w}^{2}-a_{0}^{2}}{V_{w}}
$$

where $a_{0}$ is the speed of sound in the gas ahead of the incident shock wave.

Abd-El-Fattah et al. ${ }^{10}$ experimentally observed that, irrespective of the refraction pattern, 
the incident shock and the transmitted shock can be approximated with the same piston velocity, $V_{p}$, leading to the following equality

$$
V_{p}=\frac{2}{\gamma_{I}+1} \frac{V_{i}^{2}-a_{0}^{2}}{V_{i}}=\frac{2}{\gamma_{I I}+1} \frac{V_{t}^{2}-a_{5}^{2}}{V_{t}}
$$

where $V_{i}$ and $V_{t}$ are the normal velocities of the incident and transmitted shocks, $I$ and $I I$ refer to the slow and fast phases, respectively; subscripts 0 and 5 refer to the state ahead the incident and transmitted shock, see Figure 2 a).

Using Equation 10, a relationship can be established between $V_{t}$ and $V_{i}$ as

$$
V_{t}=\frac{1}{2}\left(c+\sqrt{c^{2}+4 a_{5}^{2}}\right) \quad \text { where } \quad c=\frac{\gamma_{I I}+1}{\gamma_{I}+1} \frac{V_{i}^{2}-a_{1}^{2}}{V_{i}}
$$

\section{SHOCK REFRACTION REGIME DIAGRAM}

\section{A. Conditions at the pattern boundaries}

\section{RRE-RRR/BPR boundary}

The determination of the boundary between RRE and RRR/BPR patterns in $\chi-\omega_{i}$ space relies on the method of shock polars. By definition, a RRE is established when the pressure behind the incident shock is higher than behind the transmitted shock. As a consequence, the reflected wave has to be an expansion wave to decrease the pressure of the gas behind the incident shock and equalize it with the pressure behind the transmitted shock; this case is shown in Figure 9 a). At the limit between RRE and RRR/BPR, the incident and transmitted polars intersect each other at a value of $\delta$ corresponding to the actual deflection through the incident and transmitted shocks, see Figure $9 \mathrm{~b}$ ).

The limit between the RRE and RRR/BPR patterns is obtained as follows: for $\chi \in[0$; 1], determine by dichotomy the highest $\omega_{i}$ for which the transmitted shock polar intersects the reflected expansion polar. If there is no intersection point between the transmitted and reflected shock polars, the reflected wave is a shock (RRR) or the refraction pattern is irregular (BPR). 


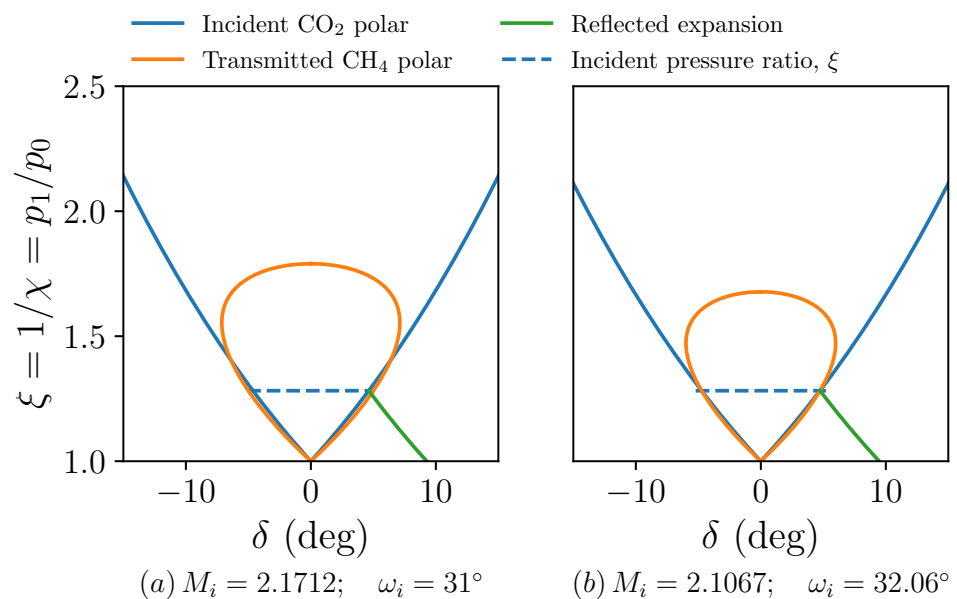

FIG. 9. Shock polar diagrams for a regular refraction with reflected expansion (RRE) structure. The plots in (a) and (b) respectively correspond to the polar diagrams away and at the limit between the RRE and RRR/BPR patterns. Conditions: $\gamma_{\mathrm{CO}_{2}}=1.288 ; \gamma_{\mathrm{CH}_{4}}=1.303 ; \mathcal{M}_{\mathrm{CO}_{2}}=44.01 \mathrm{~g} / \mathrm{mol}$; $\mathcal{M}_{\mathrm{CH}_{4}}=16.04 \mathrm{~g} / \mathrm{mol} ; \xi=1 / \chi=1.282$.

\section{RRR-BPR boundary}

The determination of the boundary between the RRR and BPR patterns also relies on the method of shock polar. By definition, the RRR pattern is established when the pressure behind the incident shock is lower than the pressure behind the transmitted shock. As a consequence, the reflected wave has to be a shock wave to increase the pressure of the gas behind the incident shock and equalize it with that behind the transmitted shock; this case is depicted in Figure $10 \mathrm{a}$ ). As seen in Figure $10 \mathrm{~b}$ ), at the limit between RRR and BPR, the reflected shock polar becomes tangent to the transmitted shock polar.

At the boundary between the RRR and BPR patterns, a tangency condition is reached between the transmitted and the reflected shock polar, so that the limit can be obtained as follows: for each $\chi \in\left[\chi_{\min } ; 1\right]$, determine by dichotomy the highest $\omega_{i}$ for which the transmitted shock polar intersects the reflected shock polar. If there is no intersection point 
between the two shock polars, it means that the refraction pattern is irregular. $\chi_{\min }$ is the value for which the $R R R \rightarrow B P R$ boundary intersects the $R R E \rightarrow R R R$ or the $R R E \rightarrow B P R$ boundary in the regime diagram. For lower values of $\chi$, the RRR $\rightarrow$ BPR boundary has no physical meaning.

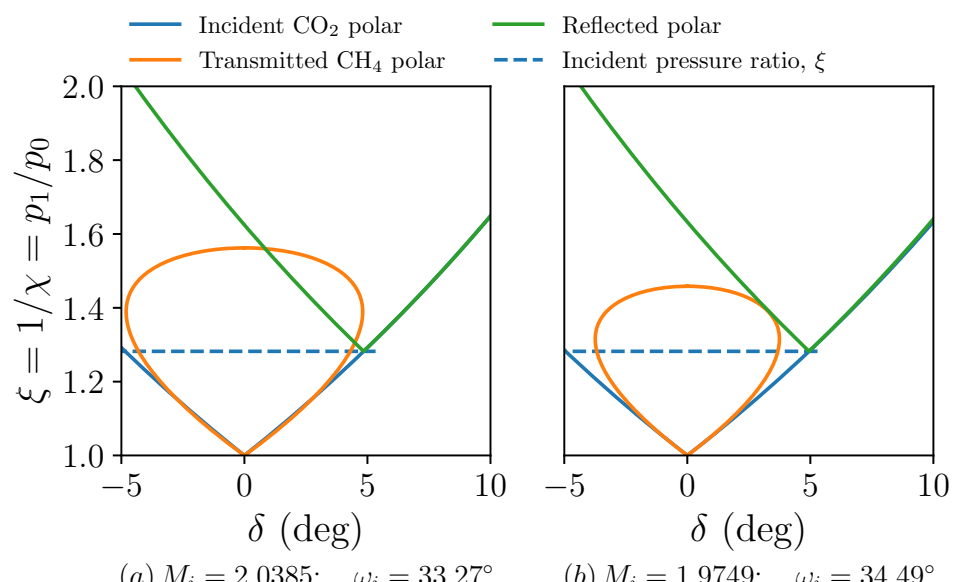

FIG. 10. Shock polar diagram for a regular refraction with reflected shock wave (RRR) structure. The plots in (a) and (b) respectively correspond to the polar diagrams away and at the limit between the RRR and BPR patterns. Conditions: $\gamma_{\mathrm{CO}_{2}}=1.288 ; \gamma_{\mathrm{CH}_{4}}=1.303 ; \mathcal{M}_{\mathrm{CO}_{2}}=44.01 \mathrm{~g} / \mathrm{mol}$; $\mathcal{M}_{\mathrm{CH}_{4}}=16.04 \mathrm{~g} / \mathrm{mol} ; \xi=1 / \chi=1.282$.

\section{BPR-FNR boundary}

The determination of the boundary between BPR and FNR patterns relies on Snell's law and the piston theory. For a BPR structure, the incident and transmitted shock waves propagate at the same velocity $(V)$ which is related to their normal velocities $\left(V_{i}\right.$ and $\left.V_{t}\right)$ by

$$
V=\frac{V_{i}}{\omega_{i}}=\frac{V_{t}}{\omega_{t}}
$$

where $\omega_{t}$ is the angle between the transmitted shock and the interface. At the transition between BPR and FNR structures, $\omega_{t}=90^{\circ}$, which enables to obtain the limit as follows: 
for $\chi \in[0 ; 1]$, determine the only $\omega_{i}$ such that $\omega_{i}=\arcsin \left(V_{i} / V_{t}\right)$, where the ratio $V_{i} / V_{t}$ is determined using Equation 10.

\section{FPR-TNR boundary}

The determination of the boundary between FPR and TNR patterns relies on shock polars and piston theory. From the FPR structure shown in Figure $3 \mathrm{c}$ ), it is seen that the gas in phase I successively passes either through the incident and reflected shocks or through the $j-$ and $k$-waves. A slip line is formed between the fluid elements traveling through these two paths. The conditions behind the incident shock and the $j$-shock are two distinct points on the same shock polar. These two points correspond to the initial conditions to calculate the reflected wave and $k$-shock polars, respectively. At the limit, the $k$-shock and the reflected wave polars are tangent to each other; this is illustrated in Figure 11.

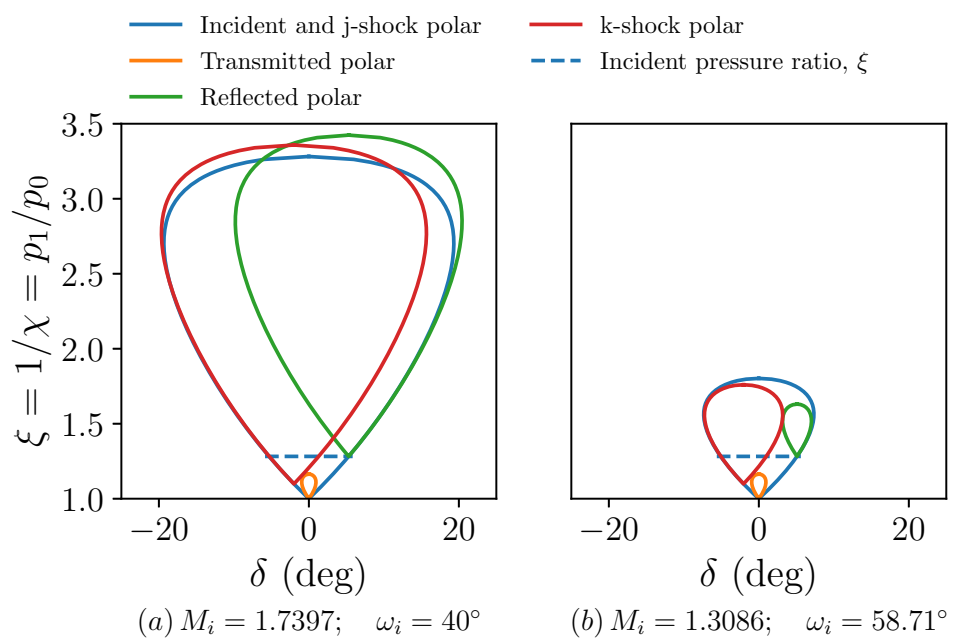

FIG. 11. Shock polar diagram for a free precursor refraction (FPR) structure. The plots in (a) and (b) respectively correspond to the polar diagrams away and at the limit between the FPR and TNR patterns. Conditions: $\gamma_{\mathrm{CO}_{2}}=1.288 ; \gamma_{\mathrm{CH}_{4}}=1.303 ; \mathcal{M}_{\mathrm{CO}_{2}}=44.01 \mathrm{~g} / \mathrm{mol} ; \mathcal{M}_{\mathrm{CH}_{4}}=16.04 \mathrm{~g} / \mathrm{mol}$; $\xi=1 / \chi=1.282$. 
The limit is obtained as follows: for $\chi \in[0 ; 1]$, determine by dichotomy the highest $\omega_{i}$ for which an intersection point exists between the $k$-shock and the reflected wave polars. The piston theory enables to calculate the $V_{t} / V_{i}$ ratio.

\section{TNR-LSR boundary}

At the transition between TNR and LSR the flow behind the incident shock wave is sonic. The boundary is obtained as follows: for $\chi \in[0 ; 1]$, determine the lowest $\omega_{i}$ for which the flow Mach number behind the incident shock, $M_{1}$, becomes less than unity.

\section{B. Validation of the regime diagram calculation}

To validate our approach to calculate the shock refraction regime diagram at a slow-fast gas-gas interface, we used the diagram provided by Abd-El-Fattah and Henderson ${ }^{11}$ for a $\mathrm{CO}_{2}-\mathrm{CH}_{4}$ interface. The comparison of their theoretical calculations with ours is shown

in Figure 12. With the exception of the FPR-TNR boundary, all the limits computed match closely the results of Abd-El-Fattah and Henderson ${ }^{11}$. As an attempt to reconcile the discrepancy observed for the FPR-TNR boundary, we used the $V_{t} / V_{i}$ experimental data provided by Abd-El-Fattah and Henderson ${ }^{11}$ shown in Figure 13. While at low shock strength the experimental $V_{t} / V_{i}$ ratio is essentially constant with $\omega_{i}$, this is not the case for stronger shocks for which $V_{t} / V_{i}$ decreases with increasing $\omega_{i}$. To improve the prediction of the FPR-TNR limit, we corrected the $V_{t} / V_{i}$ ratio applying a standard non-linear least squares method to find the best fit to the experimental results. The result is shown as a solid line in Figure 13. The limits obtained using the optimized $V_{t} / V_{i}$ are displayed in Figure 14; an overall improvement of the predicted boundary was observed despite of larger discrepancies generated in some regions of the $\chi-\omega_{i}$ space. Since the change of the $V_{t} / V_{i}$ ratio with $\omega_{i}$ depends on the strength of the shock, a large experimental data set would be required to further improve the $V_{t} / V_{i}=f\left(\omega_{i}\right)$ dependence. For our purposes (i.e., super-knock conditions) we deemed the initial discrepancies using the unmodified piston theory reasonable, and did not pursue extra improvements to the prediction of the FPR-TNR boundary.

Additional validation was carried out by comparing our results with the refraction struc- 


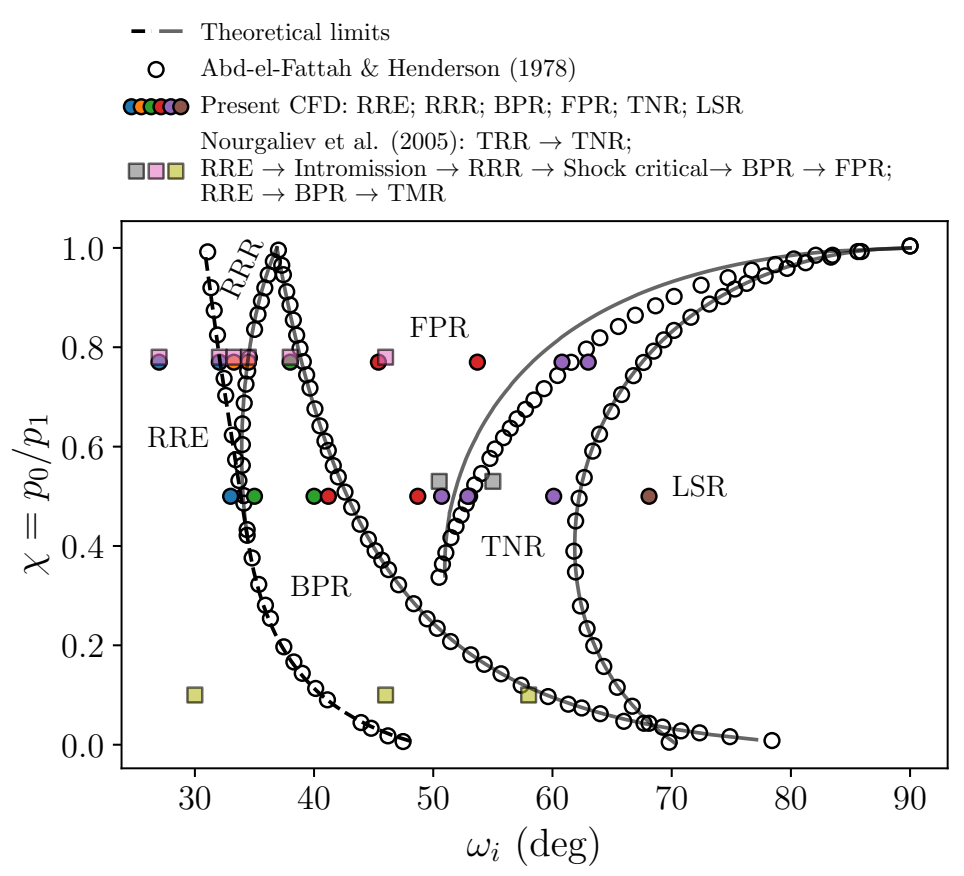

FIG. 12. Refraction regime diagram for the interaction of a planar shock and a planar oblique $\mathrm{CO}_{2}-\mathrm{CH}_{4}$ interface. Initial conditions: $p_{0}=0.1 \mathrm{MPa}$ and $T_{0}=300 \mathrm{~K}$.

tures simulated by Nourgaliev et al. ${ }^{8}$. Their numerical data were included in Figure 12 as square symbols. For a case located in the "very weak" shock group, i.e. $\chi=0.78$, Nourgaliev et al. predict the transitions $\mathrm{RRE} \rightarrow \mathrm{RRR}$ and $\mathrm{RRR} \rightarrow \mathrm{BPR}$ to occur at approximately $\omega_{i}=32^{\circ}$ and $\omega_{i}=34.5^{\circ}$, respectively. The refraction structures obtained by Nourgaliev et al. at $\omega_{i}=27,33.3,38$ and $46^{\circ}$ were respectively RRE, RRR, BPR, and FPR. These results are in quantitative agreement with our theoretical predictions and numerical results; Figure 15 shows two examples of refraction structures obtained in the "very weak" shock group. For a case located in the "weak shock" group, i.e. $\chi=0.53$, Nourgaliev et al. obtained FNR and TNR refraction patterns at $\omega_{i}=48^{\circ}$ and $\omega_{i}=52^{\circ}$, respectively. Again, this is consistent with our theoretical predictions and numerical results; Figure 16 shows two examples of refraction structures we obtained in the "weak shock" group. Finally, for a case located in the "strong shock" group, i.e. $\chi=0.18$, Nourgaliev et al. observed the 


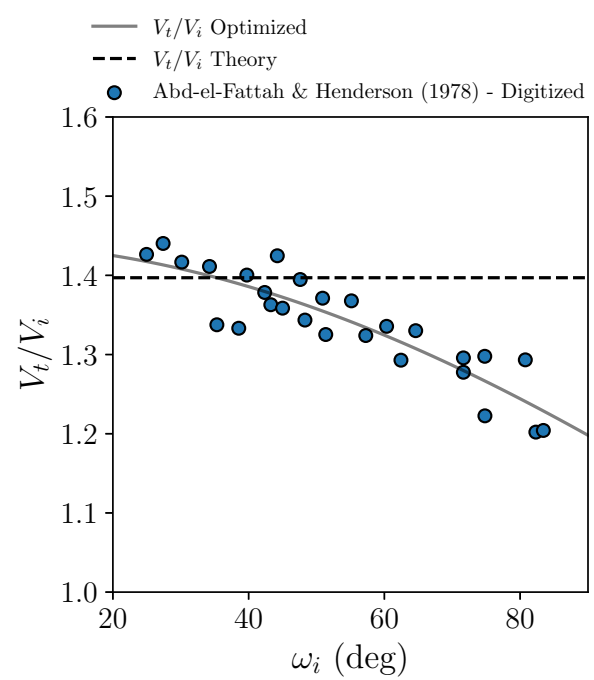

FIG. 13. Experimental and theoretical $V_{t} / V_{i}$ ratio as a function of angle of incidence for a weak shock $\left(\chi=p_{0} / p_{1}=0.53\right)$ at a slow-fast $\mathrm{CO}_{2}-\mathrm{CH}_{4}$ interface. Reproduced from Abd-El-Fattah and Henderson ${ }^{11}$.

RRE, BPR, and TNR structures for $\omega_{i}=30,46$ and $53^{\circ}$, respectively. These numerical data points also compare well with the theoretical refraction domains obtained. The agreement between our theoretical calculations and the experimental data of Abd-El-Fattah and Henderson, and between our numerical simulations and those of Nourgaliev et al., demonstrate the adequacy of our theoretical and computational methodologies.

\section{RESULTS AND DISCUSSION}

\section{A. Refraction structure under super-knock conditions}

The theoretical and numerical approaches described above were used to determine the refraction patterns under super-knock relevant conditions for a stoichiometric $\mathrm{C}_{8} \mathrm{H}_{18}$-air mixture at $T_{0}=820 \mathrm{~K}$ and $p_{0}=7.5 \mathrm{MPa}$. These conditions were chosen based on the rapid compression machine study of Wang et al. ${ }^{7}$ in which super-knock initiation was observed through the SWFID mechanism. The initial conditions are specified in Table III C 2). 


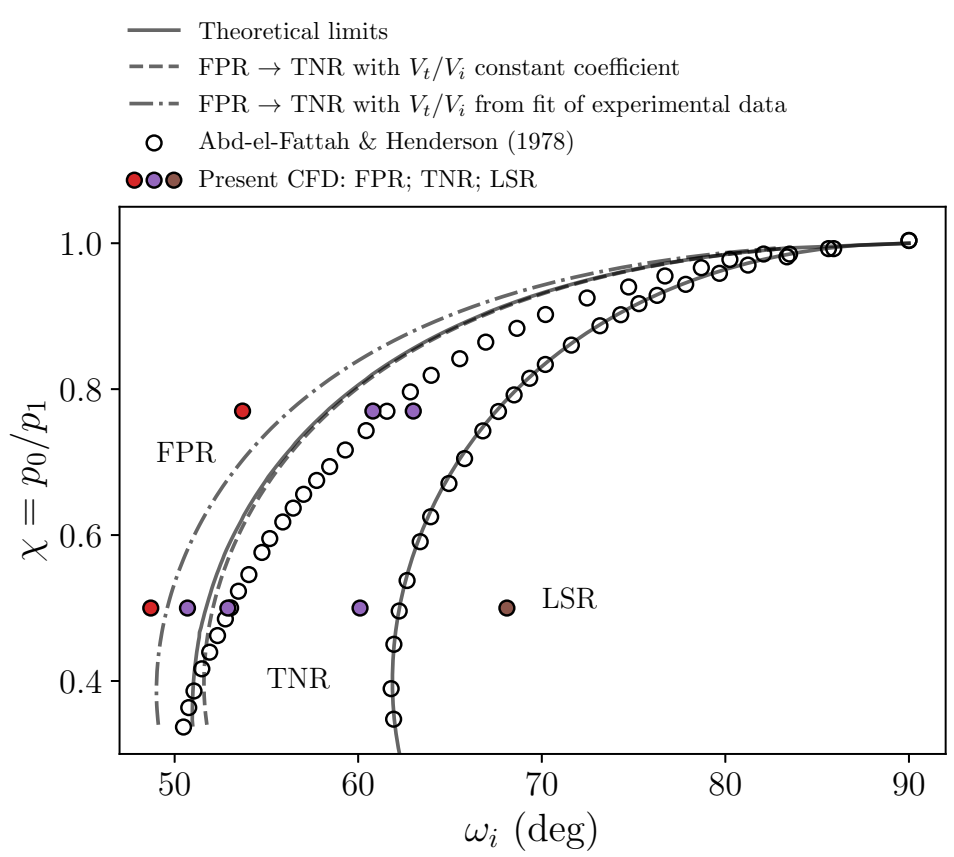

FIG. 14. Theoretical FPR-TNR limits at a slow-fast $\mathrm{CO}_{2}-\mathrm{CH}_{4}$ interface obtained for (i) a constant $V_{t} / V_{i}$ ratio, (ii) a $V_{t} / V_{i}$ ratio corrected with a constant coefficient, and (iii) a $V_{t} / V_{i}$ ratio corrected with a polynomial fit of the experimental data at $\chi=p_{0} / p_{1}=0.53$.

Three shock Mach numbers were examined for both the planar and cylindrical shock waves, $M=1.1,1.5$ and 2.0 and correspond to realistic values under super-knock conditions; note that the measurement of experimental shock velocities upon end-gas auto-ignition is not straightforward owing to the three-dimensional nature of the phenomenon.

Figure 17 shows the theoretical refraction regime diagram calculated for a fresh-burnt $\mathrm{C}_{8} \mathrm{H}_{18}$-air interface at super-knock relevant conditions. The simulation results obtained for the interaction of a planar shock and a cylindrical pocket of burnt gas are also shown in the figure. Overall, the theoretical results satisfactorily agree with the simulations results with discrepancies in $\omega_{i}$ on the order of $10^{\circ}$. Some exceptions should be pointed out: (i) the 


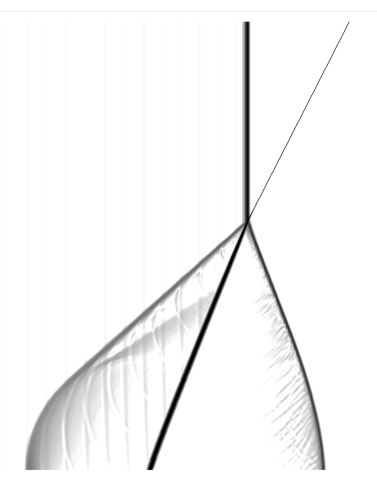

(a) RRE; $\alpha=\omega_{i}=27^{\circ}$

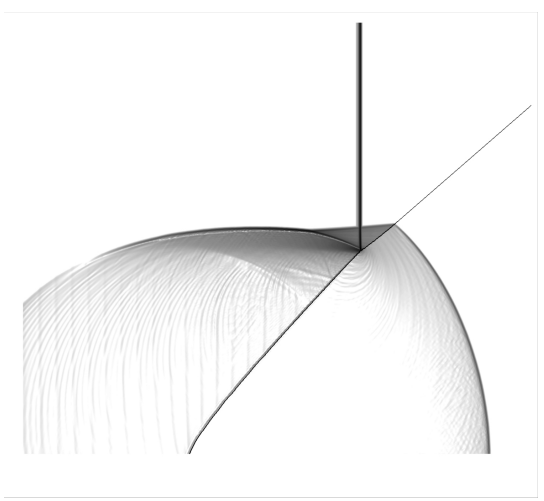

(b) FPR, $\alpha=49^{\circ} ; \omega_{i}=45.4^{\circ}$

FIG. 15. Schlieren fields of simulated RRE (a) and FPR (b) refraction patterns for a very weak shock interacting with a slow-fast $\mathrm{CO}_{2}-\mathrm{CH}_{4}$ interface. Conditions: $M=1.12(\chi=0.78) ; T_{0}=$ $300 \mathrm{~K} ; p_{0}=101 \mathrm{kPa}$.

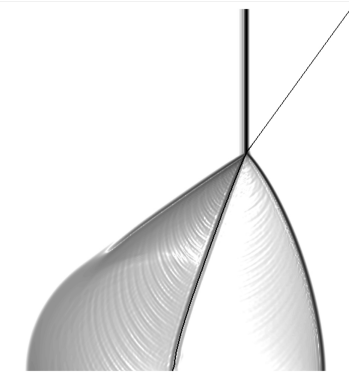

(a) BPR; $\alpha=\omega_{i}=35^{\circ}$

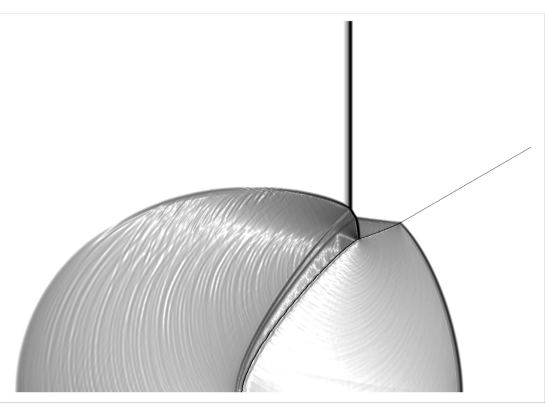

(b) TNR, $\alpha=60^{\circ} ; \omega_{i}=52.9^{\circ}$

FIG. 16. Schlieren fields of simulated BPR (a) and TNR (b) refraction patterns for a weak shock interacting with a slow-fast $\mathrm{CO}_{2}-\mathrm{CH}_{4}$ interface. Conditions: $M=1.37(\chi=0.50) ; T_{0}=300 \mathrm{~K}$; $p_{0}=101 \mathrm{kPa}$.

refraction structure observed in the simulation for $M=1.1(\chi=0.809)$ and $\omega_{i}=85.4^{\circ}$ is a FPR whereas a LSR is expected according to the theory; (ii) the numerical FNR $\rightarrow$ TNR boundary corresponds to the theoretical TNR $\rightarrow$ LSR boundary, located around $60^{\circ}$ and $70^{\circ}$, respectively. Examples of numerical schlieren images obtained are shown in Figure 18. 


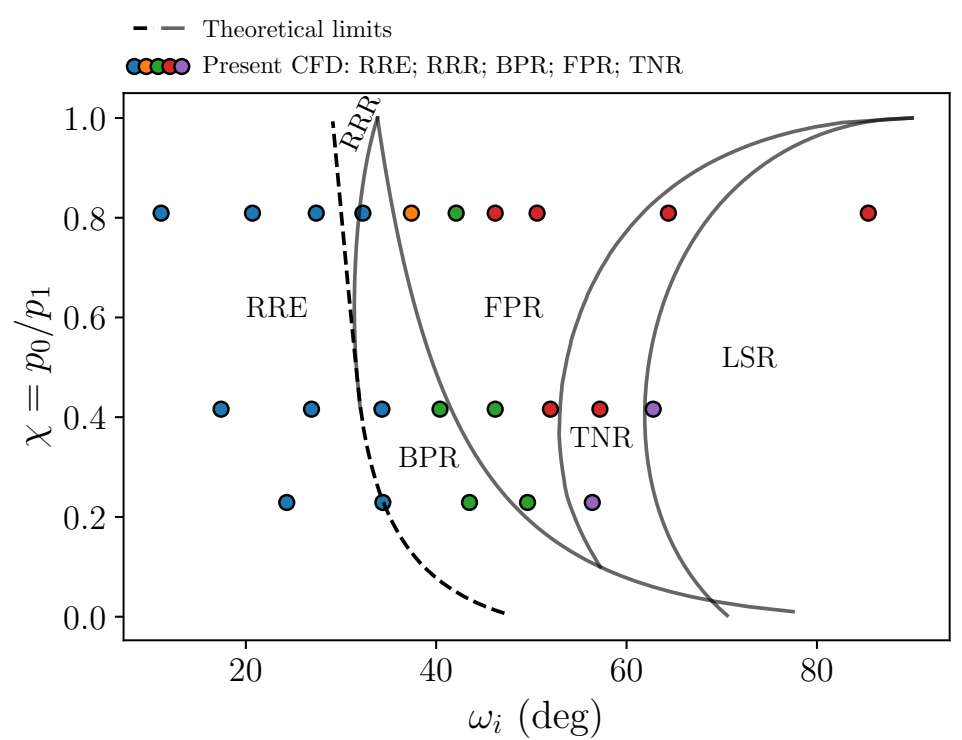

FIG. 17. Refraction regime diagram for a $\mathrm{C}_{8} \mathrm{H}_{18}$-air fresh-burnt interface at super-knock relevant conditions. Fresh mixture: stoichiometric $\mathrm{C}_{8} \mathrm{H}_{18}$-air mixture at $T_{0}=820 \mathrm{~K}$ and $p_{0}=7.5 \mathrm{MPa}$; complete initial conditions are included in Table III C 2. Simulation results obtained for a cylindrical interface and planar shock waves propagating at $M=1.1,1.5$ and $2.0(\chi=0.809,0.416$ and $0.228)$.

For all shock propagation speeds, regular patterns are observed at low angles of incidence, $\omega_{i}<40^{\circ}$. An example of RRE pattern is shown in Figure 18 a). The weakly irregular BPR patterns are typically observed in the range $\omega_{i}=40^{\circ}-45^{\circ}$. Figure $18 \mathrm{~d}$ ) and g) show examples of BPR structures. At low Mach number, $M=1.1$, only FPR patterns were captured for $\omega_{i}>45^{\circ}$, as seen in Figure $18 \mathrm{~b}$ ) and c); no TNR nor LSR patterns were observed at this Mach number. As $M$ is increased to $1.5(\chi=0.416)$ and $2.0(\chi=0.228)$, the FPR pattern appears over a restricted range of angle of incidence $\left(55<\omega_{i}<60^{\circ}\right)$. For $\omega_{i}>60^{\circ}$, TNR patterns were observed as shown in Figure $\left.\left.\left.18 \mathrm{e}\right), \mathrm{f}\right), \mathrm{h}\right)$, and i).

Similar to Figure 17, Figure 19 shows the theoretical refraction regime diagram calculated for a fresh-burnt $\mathrm{C}_{8} \mathrm{H}_{18}$-air interface at super-knock relevant conditions. However, 


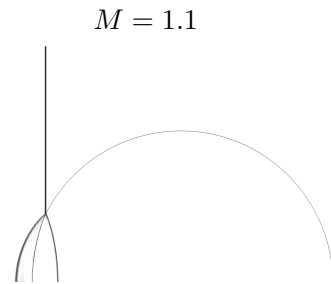

(a) RRE, $\alpha=\omega_{i}=27.4^{\circ}$

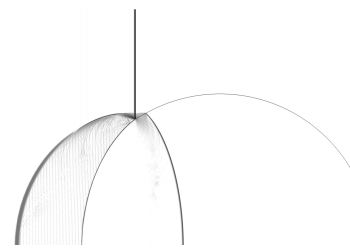

(b) FPR, $\alpha=60.9^{\circ}, \omega_{i}=59.7^{\circ}$

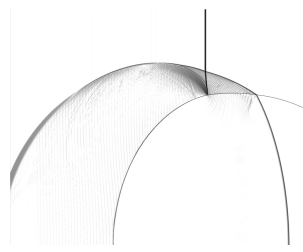

(c) FPR, $\alpha=96.3^{\circ}, \omega_{i}=82.4^{\circ}$

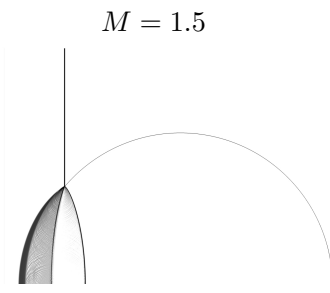

(d) BPR, $\alpha=\omega_{i}=40.4^{\circ}$

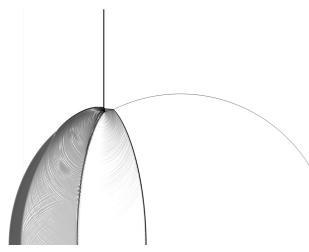

e) TNR, $\alpha=63.9^{\circ}, \omega_{i}=62.8^{\circ}$

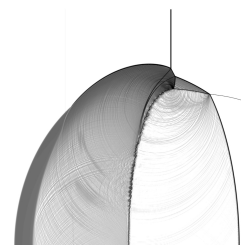

(f) $\mathrm{TNR}, \alpha=90.9^{\circ}, \omega_{i}=88.8^{\circ}$

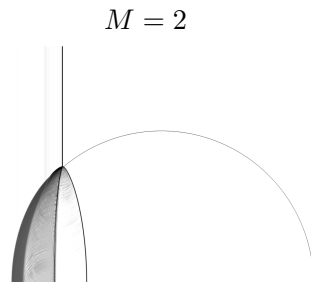

(g) BPR, $\alpha=\omega_{i}=49.6^{\circ}$

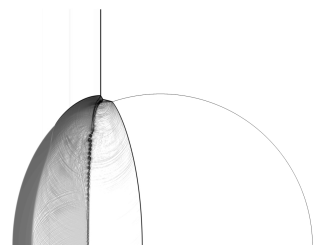

(h) TNR, $\alpha=64.9^{\circ}, \omega_{i}=63.0^{\circ}$

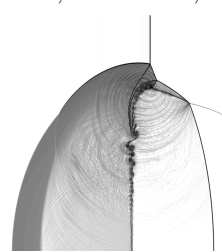

(i) TNR, $\alpha=94.9^{\circ}, \omega_{i}=83.6^{\circ}$

FIG. 18. Schlieren fields showing refraction patterns at super-knock relevant conditions. Fresh mixture: stoichiometric $\mathrm{C}_{8} \mathrm{H}_{18}$-air mixture at $T_{0}=820 \mathrm{~K}$ and $p_{0}=7.5 \mathrm{MPa}$; complete initial conditions are included in Table III C2. Simulation results obtained for a cylindrical interface and planar shock waves propagating at $M=1.1$ in (a), (b), and (c), at $M=1.5$ in (d), (e), and (f), and at $M=2.0$ in (g), (h), and (i). The inverse shock strength corresponding to $M=1.1,1.5$, and 2 are $\chi=0.809,0.416$ and 0.228 , respectively.

the simulation results were obtained by considering a cylindrical shock interacting with a cylindrical pocket of burnt gas. Comparable discrepancies to those obtained for the planar shock cases can be observed between the theoretical and numerical results. For regular patterns, the RRE domain is well predicted except that the RRR patterns position differs between the theory and the simulations. For BPR patterns, discrepancies of up to $15^{\circ}$ can be observed at low Mach numbers (i.e., high $\chi$ ). The FPR and TNR patterns are somewhat 
consistent with the theoretical limits. It is important to emphasize that the theoretical results were obtained using planar shock-planar oblique interface interactions, and that in the case at hand (cylindrical shock-cylindrical interface) extracting the angles of incidence is not straightforward; the values reported have an uncertainty of $\pm 0.2^{\circ}$. Since some of the refraction patterns exhibit similar features, it is quite difficult to clearly establish the boundaries. Moreover, the cylindrical shock slows down as it propagates which results in a lower post-shock pressure and induces an unsteady expansion of the flow behind the shock $^{39,40}$, potentially influencing the refraction patterns boundaries. These aspects could partly explain the discrepancies observed, however, further experimental and numerical work is needed to reconcile the differences. We speculate that shock curvature and associated pressure changes behind the incident shock may be the major contributors to the observed discrepancies. Finally, note that given the continuous change of the incident shock local curvature, shock velocity, and the unsteadiness behind the decaying cylindrical shock, a given refraction pattern might not have enough time to establish itself and stabilize before the flow conditions impose a drastic change of structure. This could be assessed by systematically varying the curvature of the incident shock but this was not pursued in the present work.

\section{B. Thermodynamic conditions in the refraction pattern}

The structures formed and the evolution of the thermodynamic conditions within the refraction patterns were also examined. Figure 20 shows numerical schlieren, temperature and pressure fields for $M=1.5(\chi=0.416)$ during the interaction of a cylindrical shock with a bubble of burnt gas. Regions of high pressure and temperature systematically form as a result of shock refraction, particularly in the FPR $\rightarrow$ TNR transition. This is further supported by the maximum temperature $\left(T_{\max }\right)$ and pressure $\left(p_{\max }\right)$ attained within the computational domain containing unburnt mixture for various angles of incidence, $\alpha$, displayed in Figure 21, which also shows an abrupt change as the refraction structure transits from a FPR $\rightarrow$ TNR, i.e., $\alpha=50-60^{\circ}$. The effect of incident shock curvature (planar vs. cylindrical) seems to be limited. As the Mach disk of the TNR increases in size, $T_{\max }$ and $p_{\max }$ decrease. Also shown in Figure 21 is the normalized adiabatic constant volume 


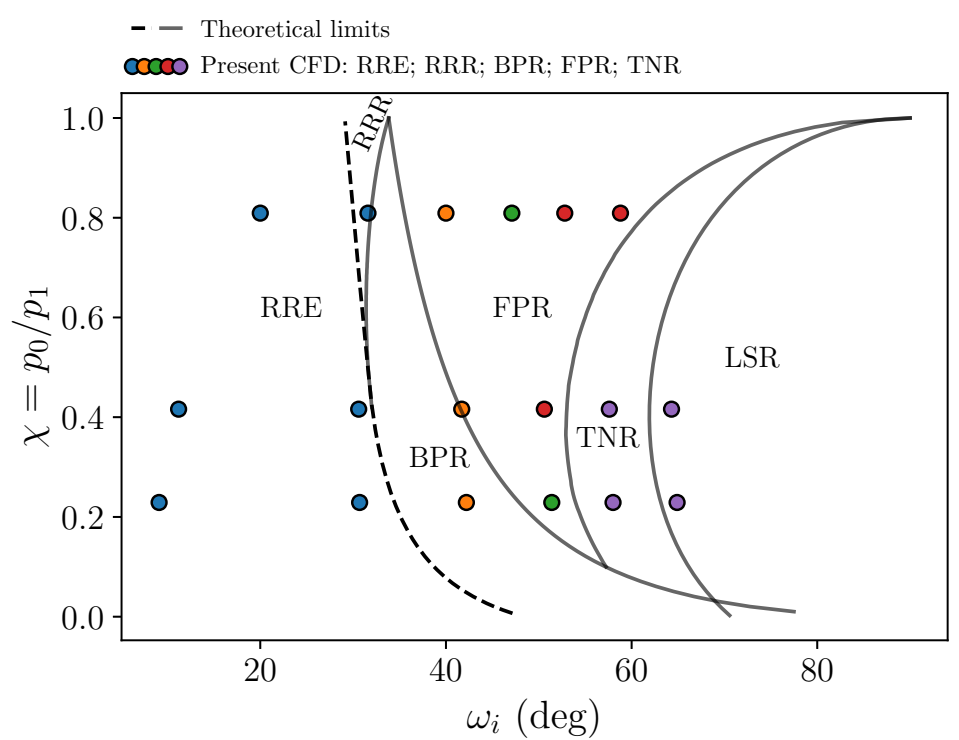

FIG. 19. Refraction regime diagram for a $\mathrm{C}_{8} \mathrm{H}_{18}$-air fresh-burnt interface at super-knock relevant conditions. Fresh mixture: stoichiometric $\mathrm{C}_{8} \mathrm{H}_{18}$-air mixture at $T_{0}=820 \mathrm{~K}$ and $p_{0}=7.5 \mathrm{MPa}$; complete conditions are included in Table III C 2. Simulation results obtained for a cylindrical interface and a cylindrical shock wave at $M=1.1,1.5$ and $2.0(\chi=0.809,0.416$ and 0.228 .)

ignition delay-time, $\tau_{\text {ind }} / \tau_{\text {ind }, 0}$, at the maximum temperature and pressure obtained for each value of $\alpha ; \tau_{\text {ind, }, 0}$ is the delay time for a $\mathrm{C}_{8} \mathrm{H}_{18}$-air mixture at $p_{0}=7.5 \mathrm{MPa}$ and $T_{0}=820 \mathrm{~K}$. The reaction model used is that of $\mathrm{Ra}$ and Reitz ${ }^{41}$, it includes 47 species and 142 reactions. For a "weak shock" $(M=1.1)$, a reduction of a factor of two is induced during the refraction process. As the Mach number is increased to $M=1.5$ and 2.0, a reduction on the order of 100 times and 10,000 times is observed during the refraction process, respectively. The formation of a hot spot with significantly higher temperature and pressure than the bulk $\mathrm{C}_{8} \mathrm{H}_{18}$-air mixture seems consistent with a local ignition in the vicinity of the shocked gas-burnt gas interface that can potentially lead to the onset of a detonation. While our simple adiabatic constant volume explosion modeling seems to yield reasonable results, we recognize that there are temporal thermodynamic and composition changes as fluid parcels travel through the refraction patterns that may favor shorter/longer ignition delay times. 
Reactive simulations may help shed some light on the actual refraction structures that may be more prone to detonation onset. In spite of this, our results support the importance of the refraction structure, particularly the FPR $\rightarrow$ TNR transition, in the ignition delay reduction. 
Schlieren
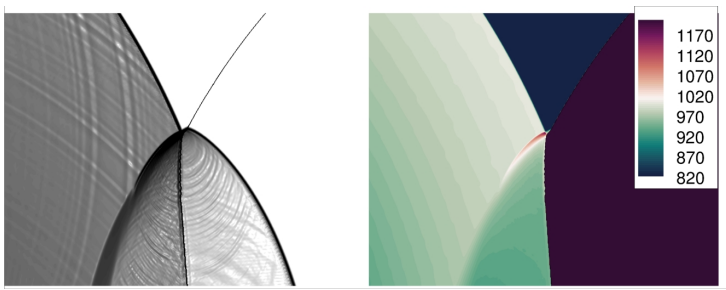

$\mathrm{FPR} ; \alpha=\omega_{i}=50.6^{\circ}$
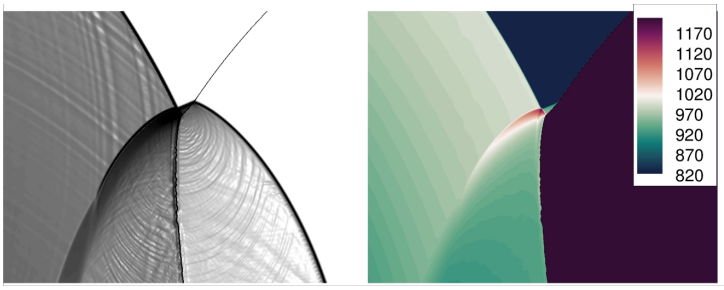

$\mathrm{TNR} ; \alpha=59.2^{\circ} ; \omega_{i}=57.0^{\circ}$
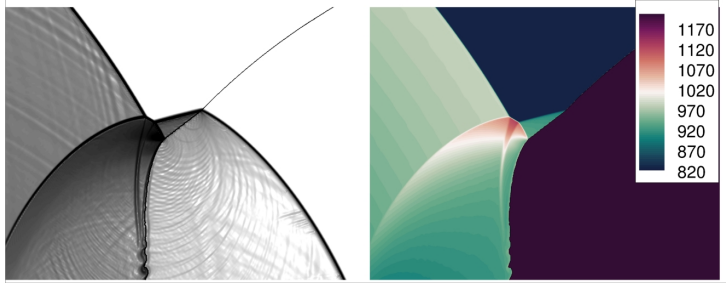

$\mathrm{TNR} ; \alpha=74.7^{\circ} ; \omega_{i}=71.2^{\circ}$
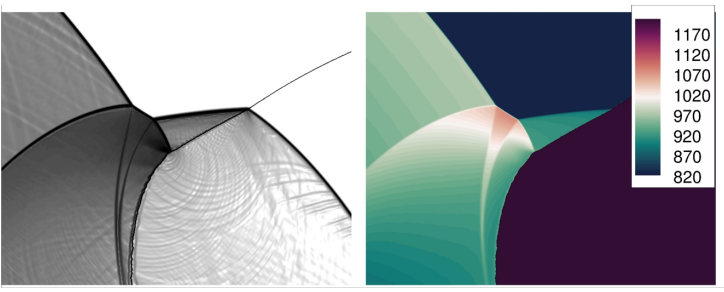

$\mathrm{TNR} ; \alpha=89.4^{\circ} ; \omega_{i}=84.7^{\circ}$
Pressure (MPa)
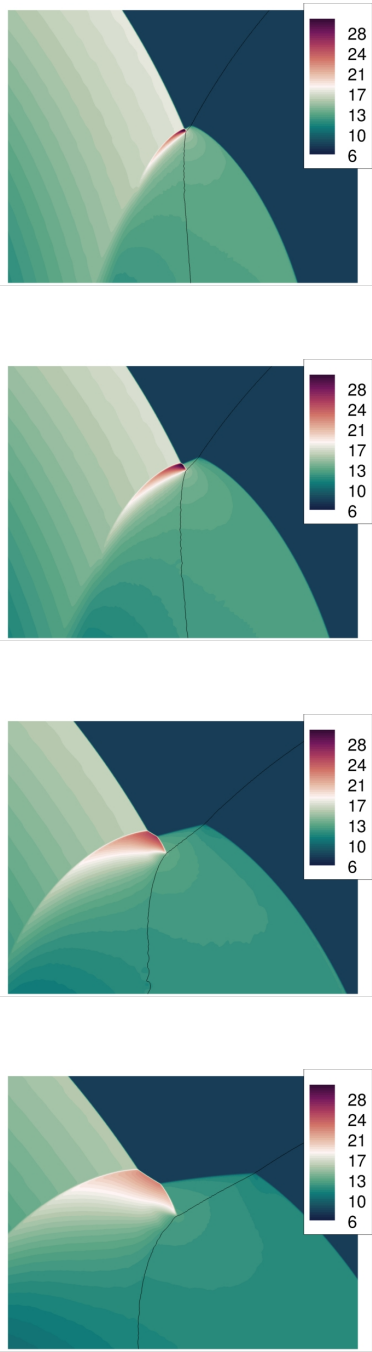

FIG. 20. Numerical schlieren, temperature and pressure fields for refraction patterns at superknock relevant conditions. Fresh mixture: stoichiometric $\mathrm{C}_{8} \mathrm{H}_{18}$-air mixture at $T_{0}=820 \mathrm{~K}$ and $p_{0}=7.5 \mathrm{MPa}$; complete conditions given in Table IIIC2. Simulation results obtained for a cylindrical interface and a cylindrical shock wave propagating at $M=1.5(\chi=0.416)$. 

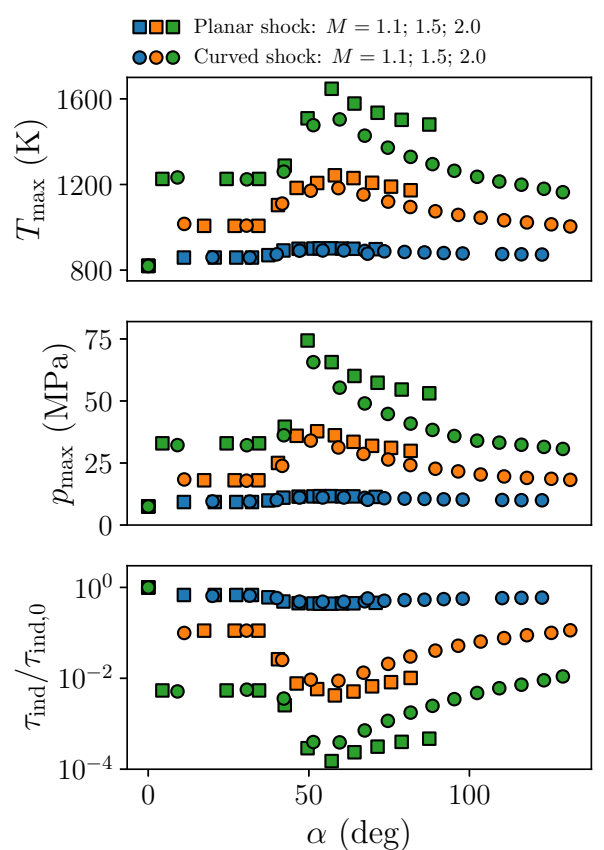

FIG. 21. Evolution of the maximum temperature, $T_{\max }$, and pressure, $p_{\max }$, within the computational domain containing unburnt mixture, and of the normalized constant volume ignition delay-time, $\tau_{\text {ind }} / \tau_{\text {ind, } 0}$, as a function of angle of incidence, $\alpha$, during shock wave refraction. Fresh mixture: stoichiometric $\mathrm{C}_{8} \mathrm{H}_{18}$-air at $T_{0}=820 \mathrm{~K}$ and $p_{0}=7.5 \mathrm{MPa}$; complete conditions given in Table III C 2. Simulation results obtained for planar/cylindrical shock-curved interface interactions.

\section{CONCLUSION}

The refraction patterns at a slow-fast gas-gas interface were theoretically and numerically investigated considering conditions relevant to super-knock in SI-ICE. Our theoretical and numerical approaches were first validated using previous work ${ }^{8,11}$ for a planar shock-planar oblique $\mathrm{CO}_{2}-\mathrm{CH}_{4}$ interface interactions. Our methodology was then applied to a $\mathrm{C}_{8} \mathrm{H}_{18}$-air fresh-burnt mixture interface at super-knock relevant conditions. While the theoretical refraction patterns were observed to satisfactorily agree with those numerically predicted for a planar shock and a curved interface, the theoretical refraction regime diagram obtained for cylindrical shock-curved interface interactions showed discrepancies. These discrepancies 
possibly arise because of the shock speed decay due to the diverging nature of the curved incident shock; additional simulations are needed to confirm the role of shock curvature on the refraction patterns. Our numerical results show that a peak of temperature and pressure forms as the refraction structure transits from a FPR to a TNR, inducing a significant reduction of the ignition delay-time which can potentially lead to a local ignition and subsequent detonation onset. Future work will focus on performing reactive simulations of the FPR-TNR transition to assess the possibility of detonation initiation. Investigating potential three-dimensional effects on the expected refraction patterns at super-knock relevant conditions may be an avenue worth exploring.

\section{ACKNOWLEDGMENTS}

This paper was partly supported by the opening project of State Key Laboratory of Explosion Science and Technology (Beijing Institute of Technology). The opening project number is KFJJ20-15M.

\section{CONFLICT OF INTEREST}

The authors have no conflicts to disclose.

\section{DATA AVAILABILITY}

The data that support the findings of this study are available from the corresponding author upon reasonable request. 


\section{REFERENCES}

${ }^{1}$ Wang Z., Li F., Wang Y., A generalized kinetic model with variable octane number for engine knock prediction, Fuel, 188, 489-499 (2017).

${ }^{2}$ Qi Y., Wang Z., Wang J., He X., Effects of thermodynamic conditions on the end gas combustion mode associated with engine knock, Combustion and Flame, 62, 4119-4128 (2015).

${ }^{3}$ Wang Z., Liu H., Reitz, R., Knocking combustion in spark-ignition engines, Progress in Energy and Combustion Science, 61, 78-112 (2017)

${ }^{4}$ Netzer C., Seidel L., Pasternak M., Lehtiniemi H., Perlman C., Ravet F., Mauss, F., Threedimensional computational fluid dynamics engine knock prediction and evaluation based on detailed chemistry and detonation theory, International Journal of Engine Research, 19(1), 33-44 (2018).

${ }^{5}$ Wang Z., Qi Y., He X.X., Wang J., Shuai S., Law C.K., Analysis of pre-ignition to superknock: Hotspot-induced deflagration to detonation, Fuel, 144, 222-227 (2015).

${ }^{6}$ Wang Z., Qi Y., Liu H., Zhang P., He X., Wang J. Shock wave reflection induced detonation (SWRID) under high pressure and temperature condition in closed cylinder, Shock Waves, 26, 687-691 (2016).

${ }^{7}$ Wang Y., Xiang S., Qi Y., Mével R., Wang, Z., Shock wave and flame front induced detonation in rapid compression machine, Shock Waves, 28, 1109-1116 (2018).

${ }^{8}$ Nourgaliev R.R., Sushchikh S.Y., Dinh T.N., Theofanous T.G., Shock wave refraction patterns at interfaces, International Journal of Multiphase Flow, 31, 969-995, (2005).

${ }^{9}$ Han Z., Yin X., Shock Dynamics, Springer, Netherlands (1993).

${ }^{10}$ Abd-El-Fattah A.M., Henderson L.F., Lozzi A., Precursor shock waves at a slow-fast gas interface, Journal of Fluid Mechanics, 76, 157-176 (1976).

${ }^{11}$ Abd-El-Fattah A.M., Henderson L.F., Shock waves at a slow-fast gas interface, Journal of Fluid Mechanics, 89, 79-95 (1978).

${ }^{12}$ Henderson L.F., On the refraction of shock waves, Journal of Fluid Mechanics, 198, 365-386 (1989).

${ }^{13}$ Henderson L.F., The refraction of a plane shock wave at a gas interface, Journal of Fluid Mechanics, 26, 607-637 (1966). 
${ }^{14}$ Henderson L.F., Colella P., Puckett E.G., On the refraction of shock waves at a slow-fast gas interface, Journal of Fluid Mechanics, 224, 1-27 (1991).

${ }^{15}$ Zeng S., Takayama K., On the refraction of shock wave over a slow-fast gas interface, Acta Astronautica, 38(11), 829-838 (1996).

${ }^{16}$ Hass J.-F., Sturtevant B., Interaction of weak shock waves with cylindrical and spherical gas inhomogeneities, Journal of Fluid Mechanics, 181, 41-76 (1987).

${ }^{17}$ Samtaney R., Pullin D.I., Self-similar hypervelocity shock interactions with oblique contact discontinuities. Shock Waves, 8, 299-310, (1998).

${ }^{18}$ Zhang W., Zou, L., Zheng X., Wang B., Numerical study on the interaction of a weak shock wave with an elliptic gas cylinder, Shock Waves, 29, 273-284 (2019).

${ }^{19}$ Martinez-Ruiz D., Huete C., Martinez-Ferrer P.J., Mira D., Specific heat effects in twodimensional shock refractions, Shock Waves, 31, 1-17 (2021).

${ }^{20}$ Kundu A., Numerical simulation of a shock-helium bubble interaction, Shock Waves, 31, 19-30 (2021).

${ }^{21}$ Zhai Z., Dong P., Si T., Luo, X., The Richtmyer-Meshkov instability of a "V" shaped air/helium interface subjected to a weak shock. Physics of Fluids, 28 \# 082104 (2016).

${ }^{22}$ Igra D., Igra O., Numerical investigation of the interaction between a planar shock wave with square and triangular bubbles containing different gases. Physica of Fluids, 30, \#056104 (2018).

${ }^{23}$ Singh S., Role of Atwood number on ow morphology of a planar shock-accelerated square bubble: A numerical study. Physics of Fluids, 32, \#126112 (2020).

${ }^{24}$ Chen J., Qu F., Wu X., Wang Z., Bai J., Numerical study of interactions between shock waves and a circular or elliptic bubble in air medium. Physics of Fluids, 33, \#043301 (2021).

${ }^{25}$ Zhai Z., Li W., Si T., Luo X., Yang J., Lu X., Refraction of cylindrical converging shock wave at an air/helium gaseous interface. Physics of Fluids, 29, \#016102, (2017).

${ }^{26}$ Haehn N.,Ranjan D., Weber C., Oakley J., Rothamer D., Bonazza, R., Reacting shock bubble interaction. Combustion and Flame, 159, 1339-1350 (2012).

${ }^{27}$ Diegelmann F., Hickel S., Adams N.A., Shock Mach number influence on reaction wave types and mixing in reactive shock-bubble interaction. Combustion and Flame, 174, 85-99 (2016). 
${ }^{28}$ Diegelmann F., Tritschler V., Hickel S., Adams N., On the pressure dependence of ignition and mixing in two-dimensional reactive shock-bubble interaction. Combustion and Flame, 163, 414-426 (2016).

${ }^{29}$ Diegelmann F., Hickel S., Adams N.A., Three-dimensional reacting shock-bubble interaction. Combustion and Flame, 181, 300-314 (2017).

${ }^{30}$ Thomas G., Bambrey R., Brown C., Experimental observations of flame acceleration and transition to detonation following shock-flame interaction. Combustion Theory and Modeling, 5, 573-594 (2001).

${ }^{31}$ Bakalis G., Tang-Yuk K.C., Mi X., Nikiforakis N., Ng H.D., Numerical simulation of deflagration-to-detonation transition via shock-multiple flame kernels interactions. Computers \& Mathematics with Applications, 83, 111-126 (2021).

${ }^{32}$ Ben-Dor G., Shock Wave Reflection Phenomena, Springer-Verlag, New-York (1992).

${ }^{33}$ Jahn R.G., The refraction of shock waves at a gaseous interface, Journal of Fluid Mechanics, 1, 457-489 (1956).

${ }^{34}$ Hartmann D., Meinke M., Schröder W., The constrained reinitialization equation for level set methods, Journal of Computational Physics, 229(5), 1514-1535 (2010).

${ }^{35}$ Toro E.F., The HLL and HLLC Riemann solvers, in Riemann solvers and numerical methods for fluid dynamics, Springer, 315-344 (2009).

${ }^{36}$ Liu X.-D., Osher S., Chan T., Weighted essentially non-oscillatory schemes, Journal of Computational Physics, 115(1), p 200-212 (1994).

${ }^{37}$ Gottlieb S., Shu C.-W., Total variation diminishing Runge-Kutta schemes, Mathematics of Computation, 67(221), 73-85 (1998).

${ }^{38}$ Abgrall R., Karni S., Ghost-fluids for the poor: a single fluid algorithm for multifluids, in Hyperbolic problems: theory, numerics, applications, Springer, 1-10 (2001).

${ }^{39}$ Eckett C.A., Quirk J.J., Shepherd J.E., The role of unsteadiness in direct initiation of gaseous detonations. Journal of Fluid Mechanics, 421, p 147-183 (2000).

${ }^{40}$ Faghih M., Mevel R., He Y., Chen Z., Effect of 2-step energy release on direct detonation initiation by a point energy source in a rich $\mathrm{H}_{2} \mathrm{NO}_{2} / \mathrm{N}_{2} \mathrm{O}_{4}$ mixture. Combustion and Flame, 222, 317-325 (2020).

${ }^{41}$ Ra Y., Reitz R.D., A reduced chemical kinetic model for IC engine combustion simulations with primary reference fuels. Combustion and Flame, 155(4), 713-738 (2008). 


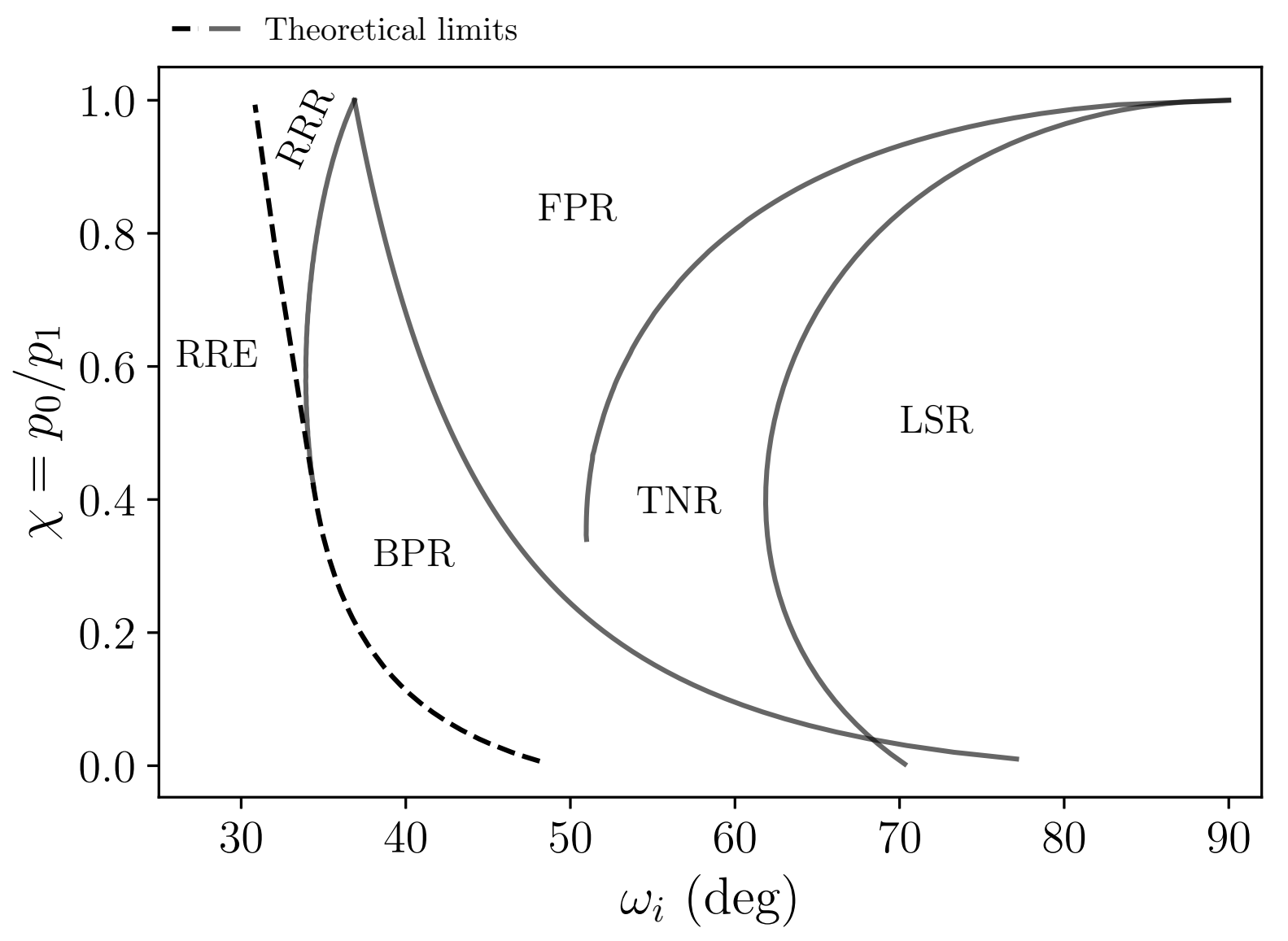




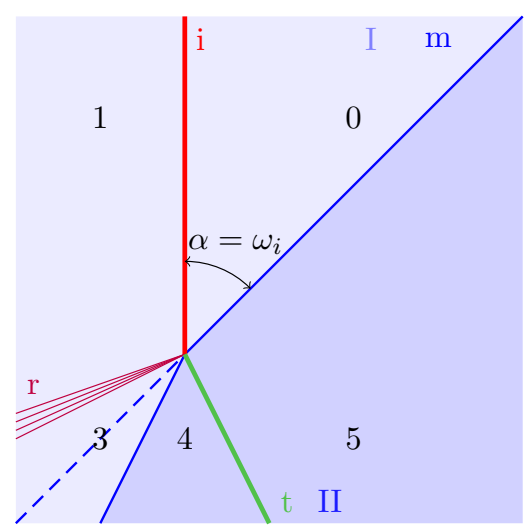

Figure 1: Wave pattern for Regular Refraction with a Reflected shock (RRR). 


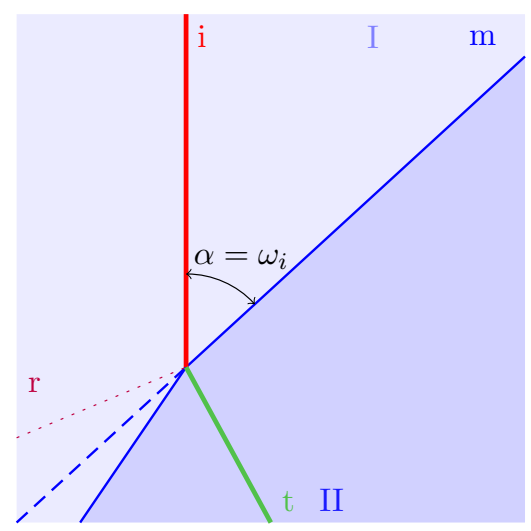

Figure 2: Regular refraction with Mach line

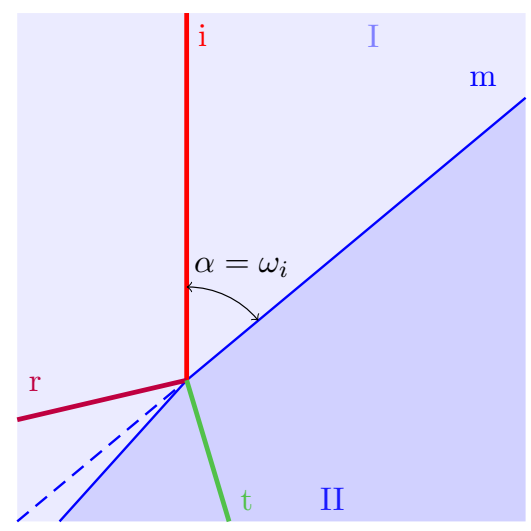

Figure 3: RRR refraction

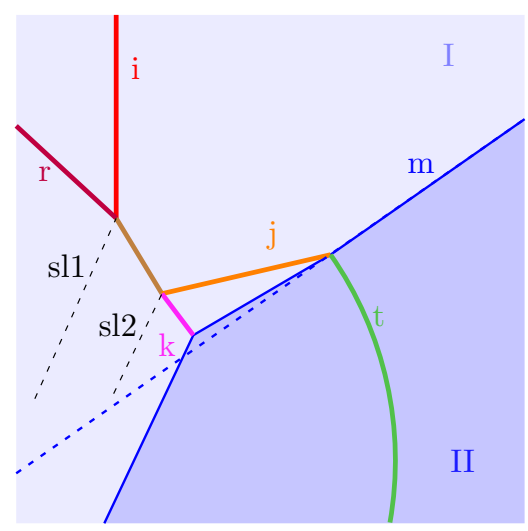

Figure 4: Simplified representation of TNR 


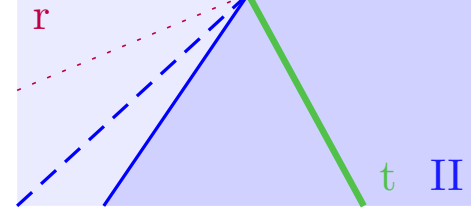

Figure 2: Regular refraction with Mach line

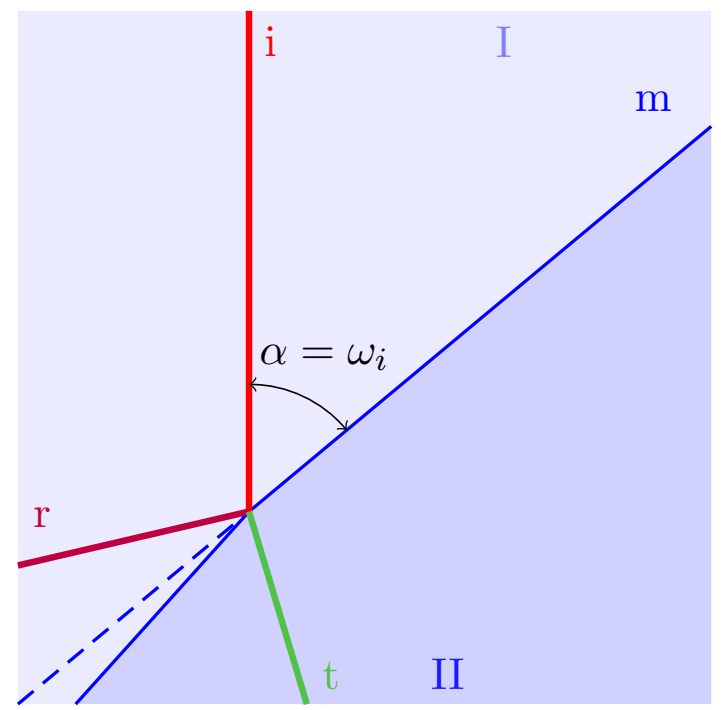

Figure 3: RRR refraction

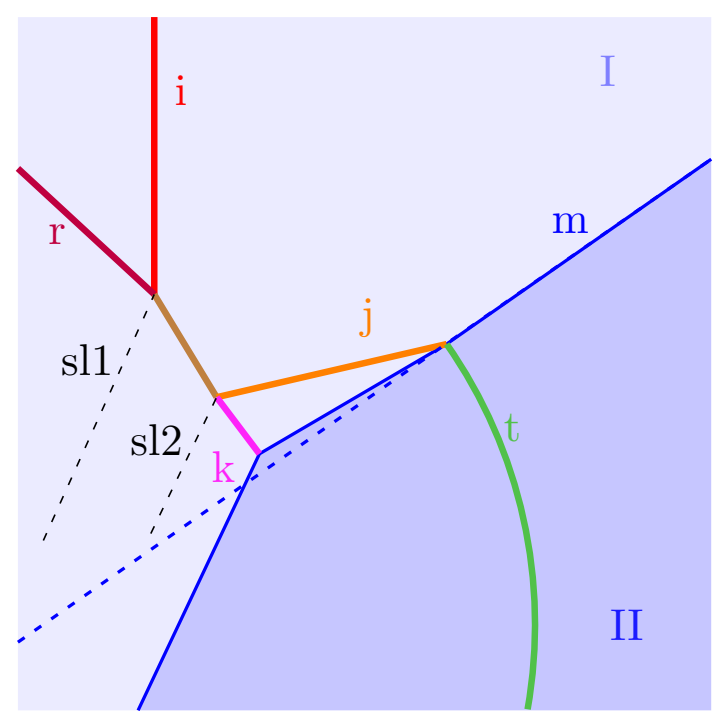

Figure 4: Simplified representation of TNR 

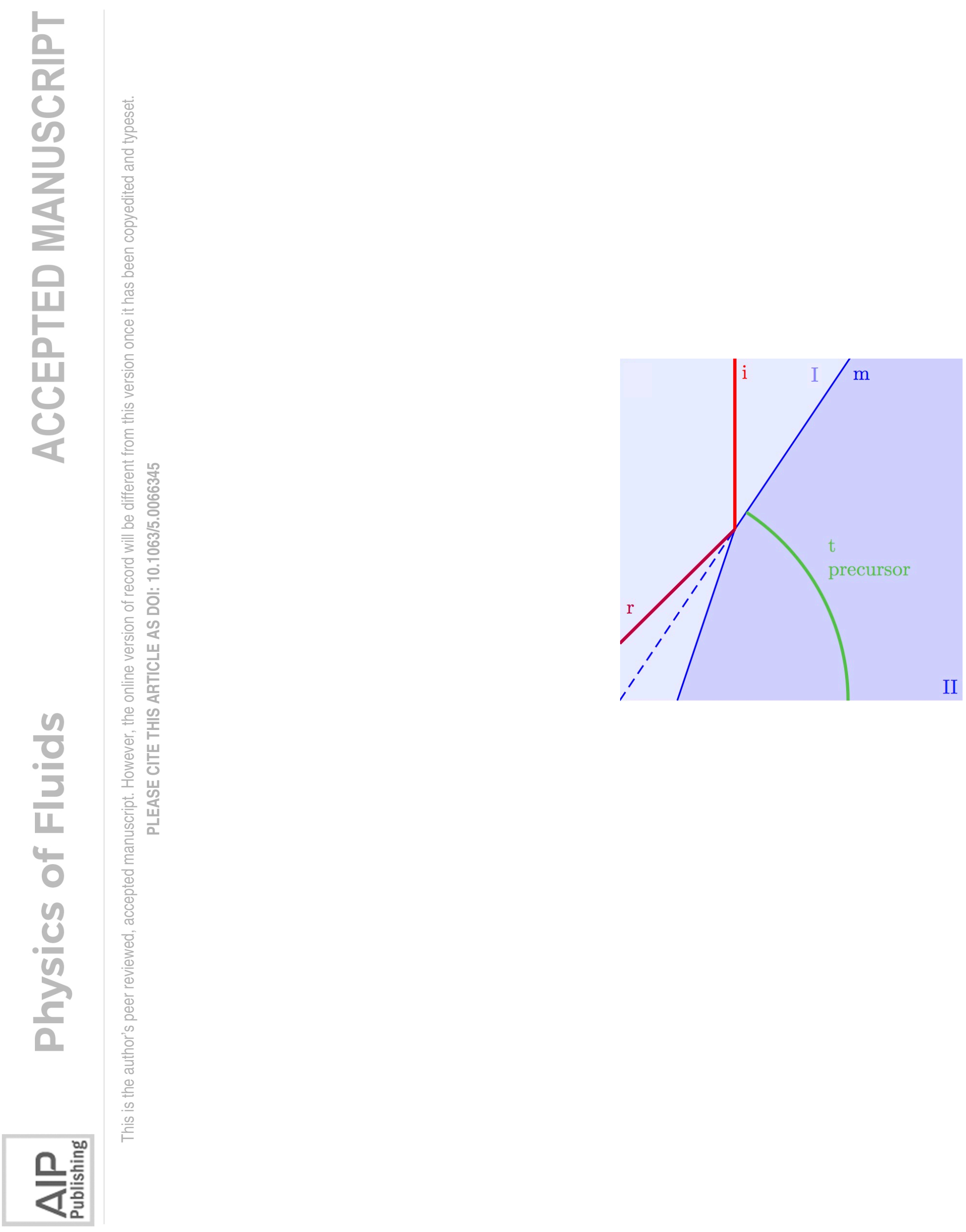


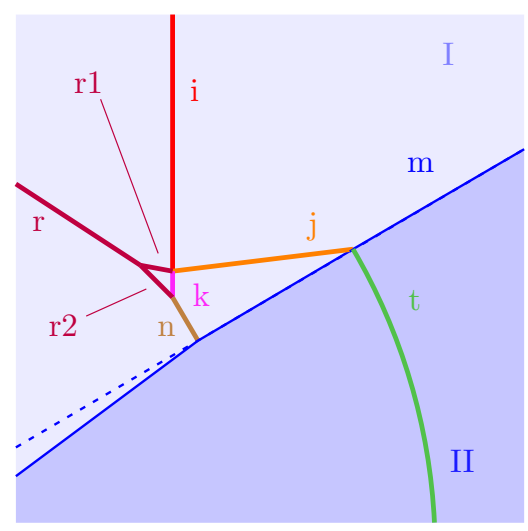

Figure 5: Simplified representation of FNR

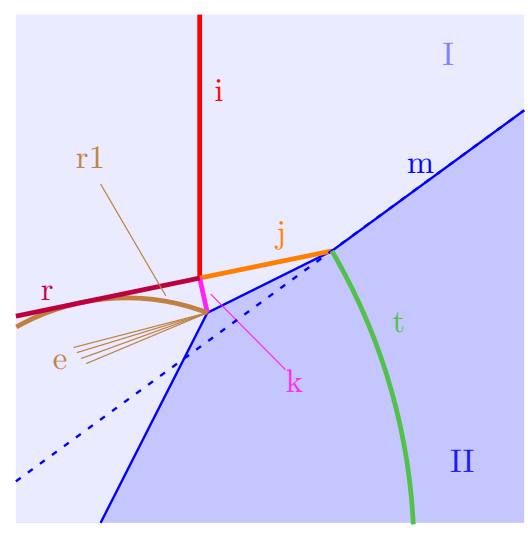

Figure 6: Simplified representation of FPR

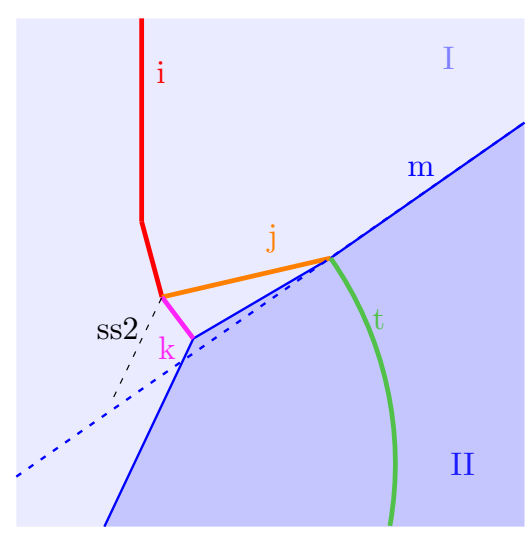

Figure 7: Simplified representation of LNR 


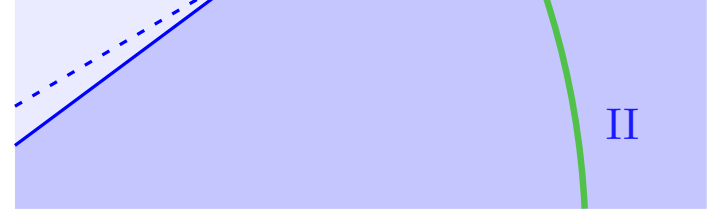

Figure 5: Simplified representation of FNR

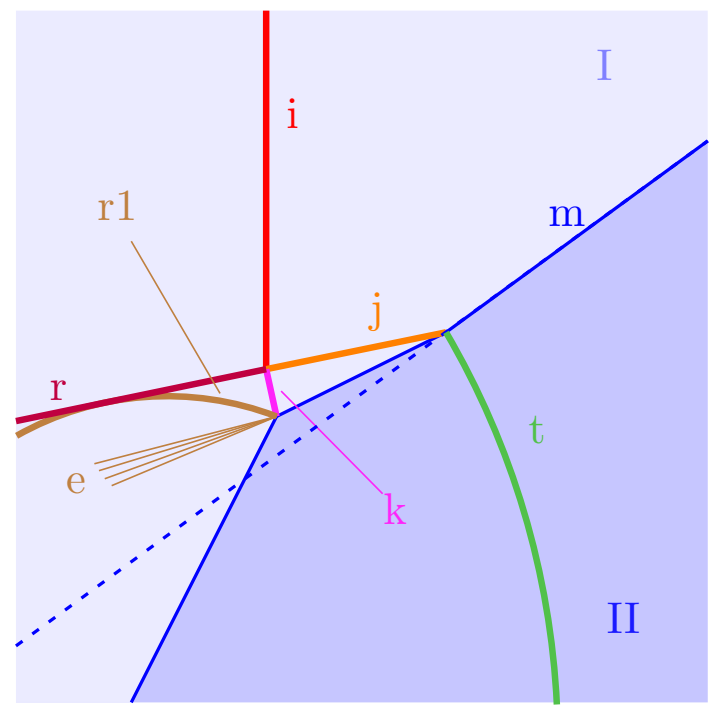

Figure 6: Simplified representation of FPR

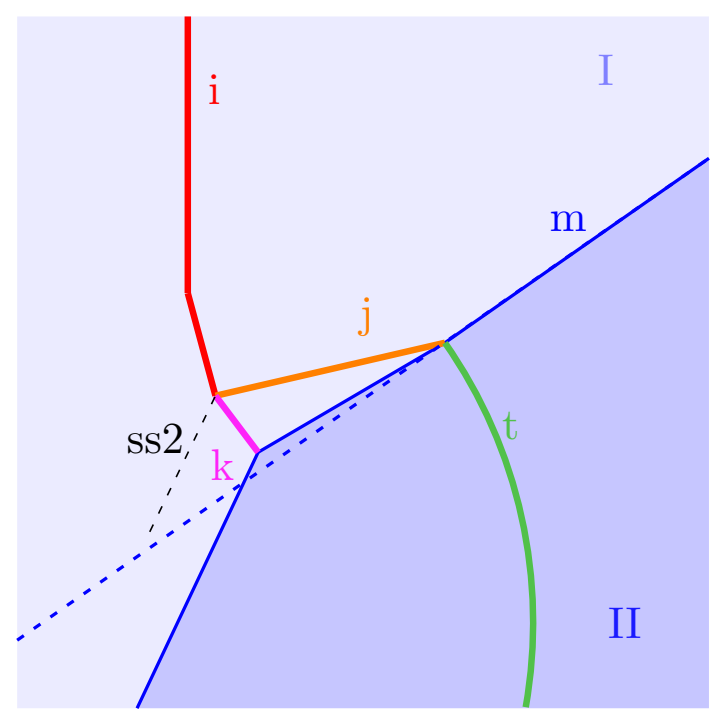

Figure 7: Simplified representation of LNR 


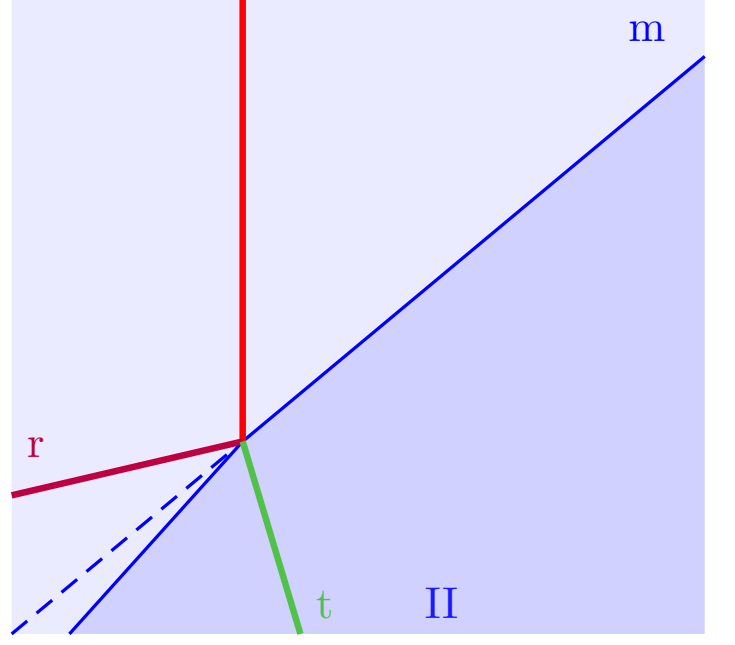

Figure 3: RRR refraction

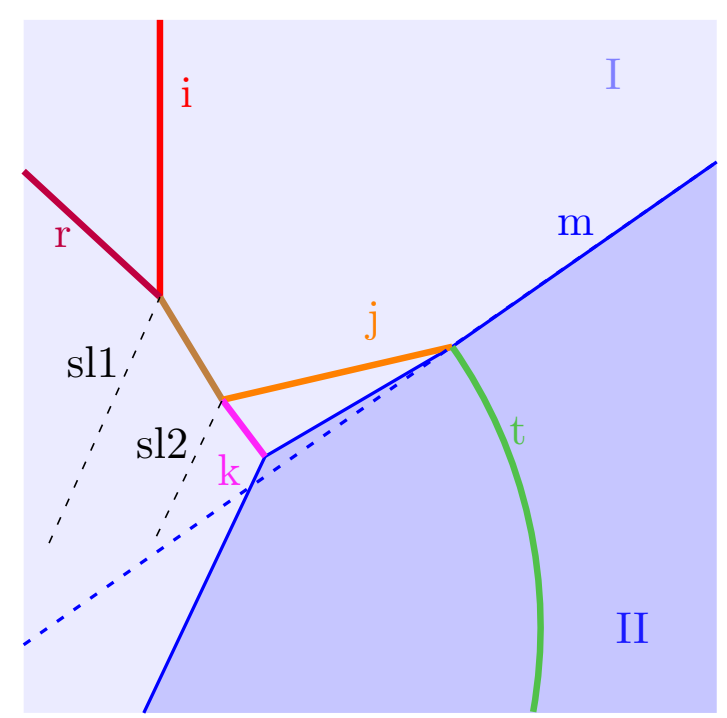

Figure 4: Simplified representation of TNR 


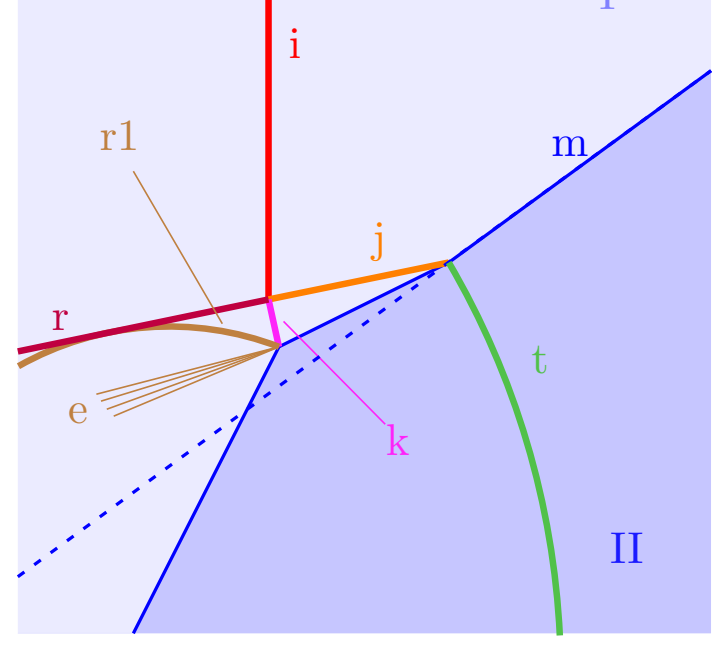

Figure 6: Simplified representation of FPR

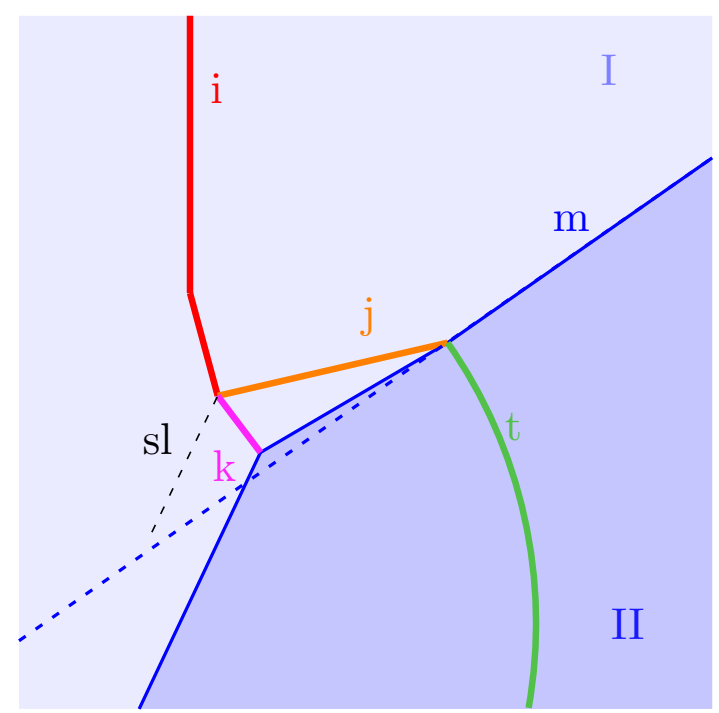

Figure 7: Simplified representation of LSR 


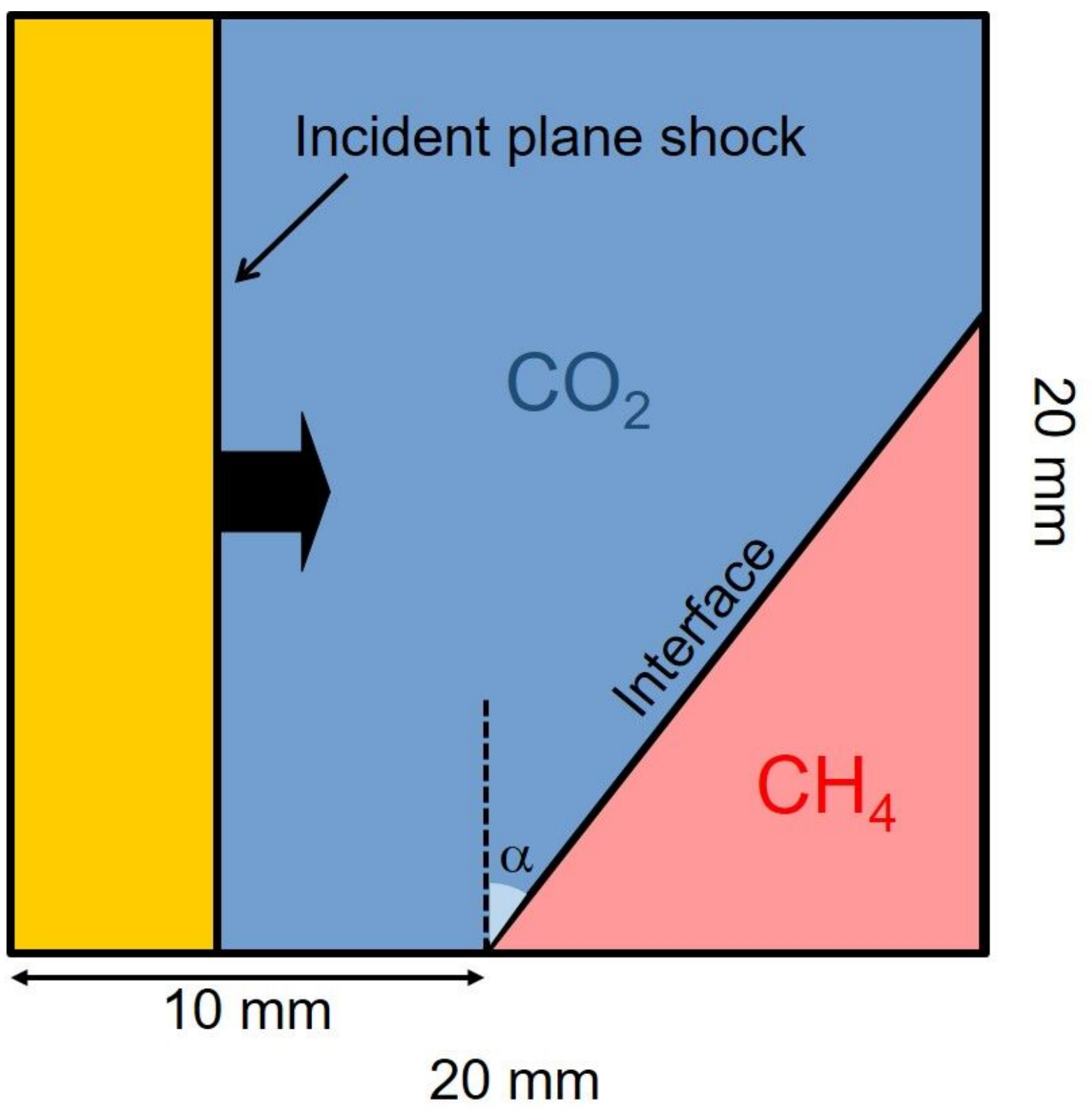

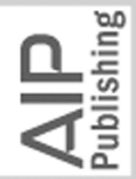



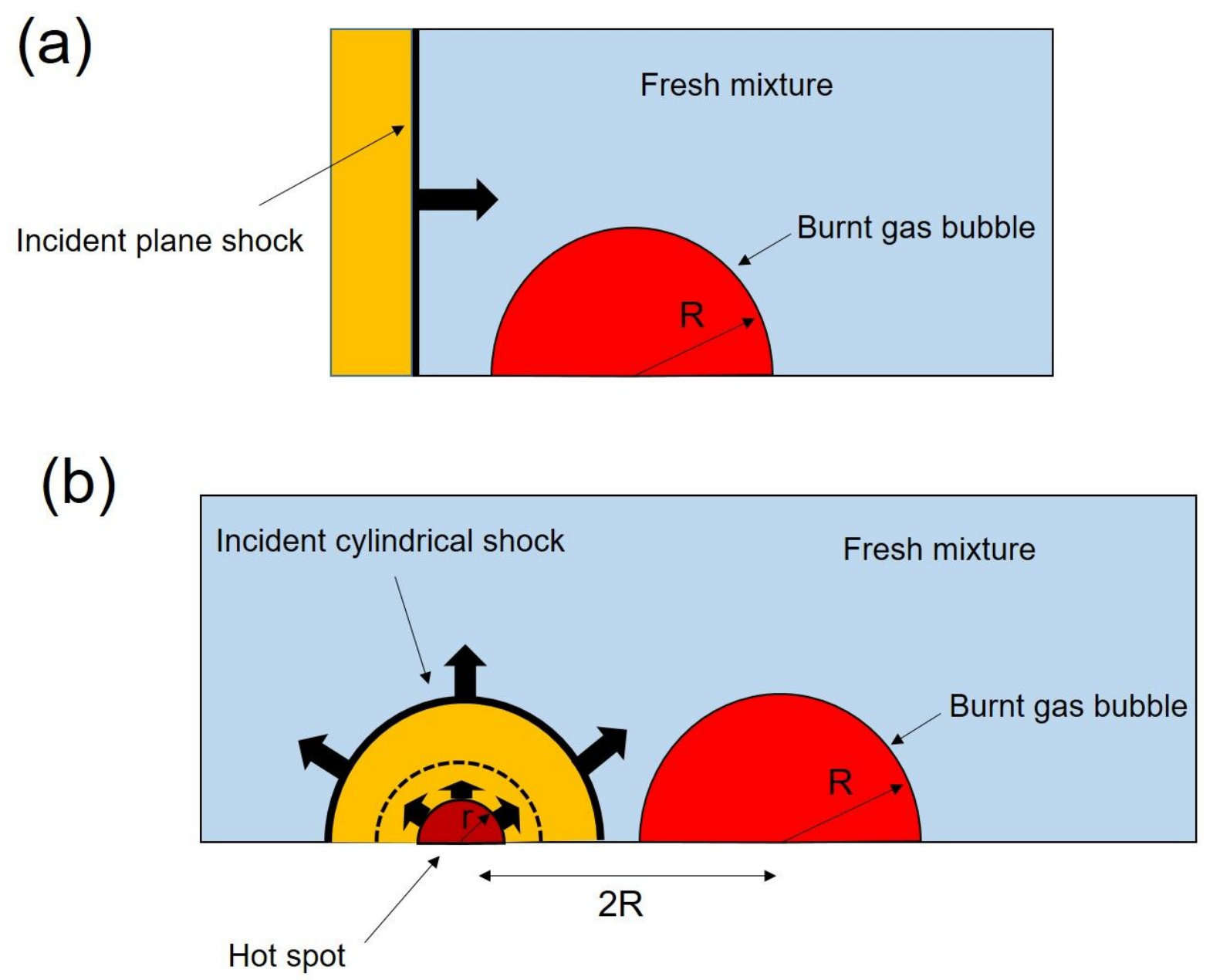


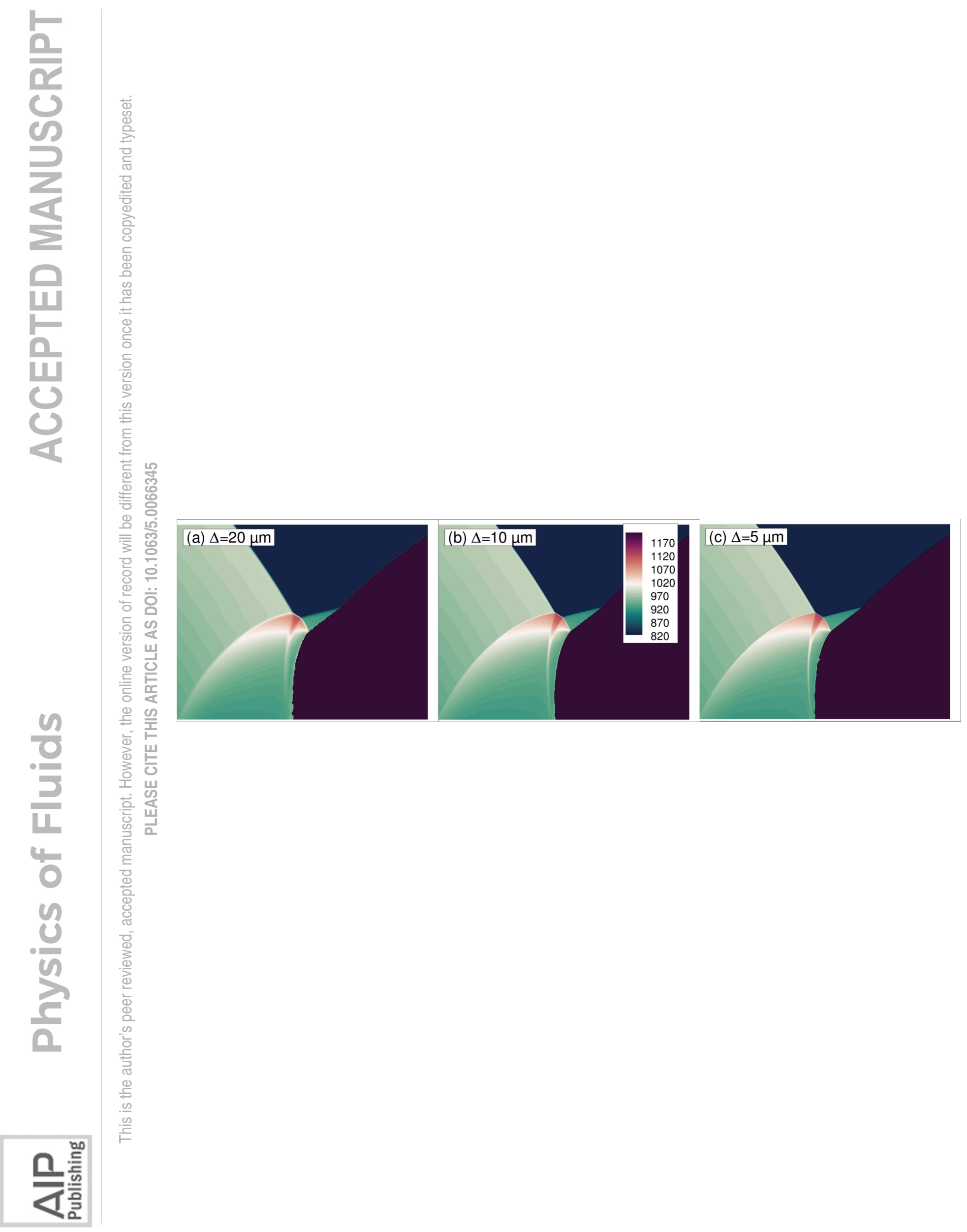



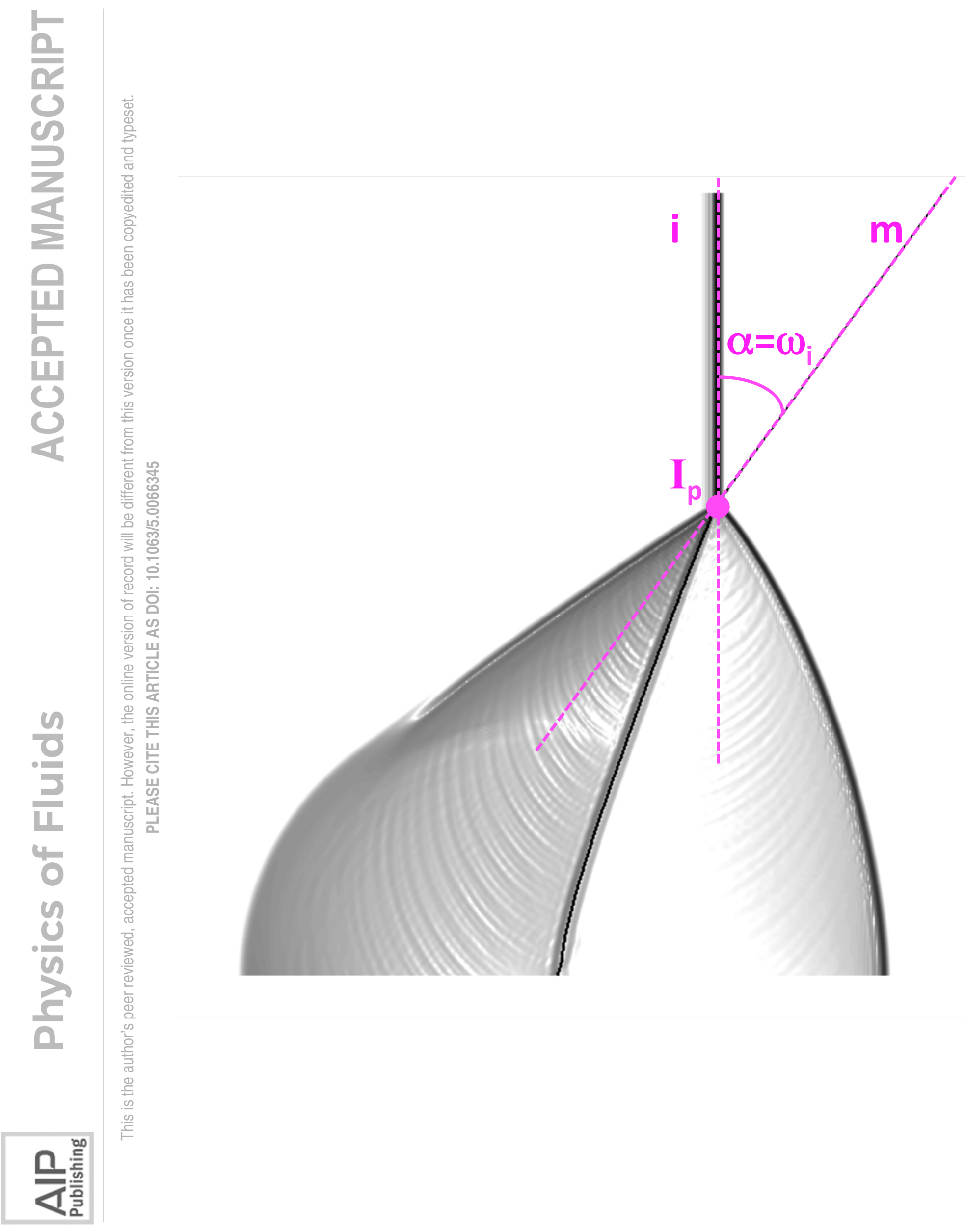


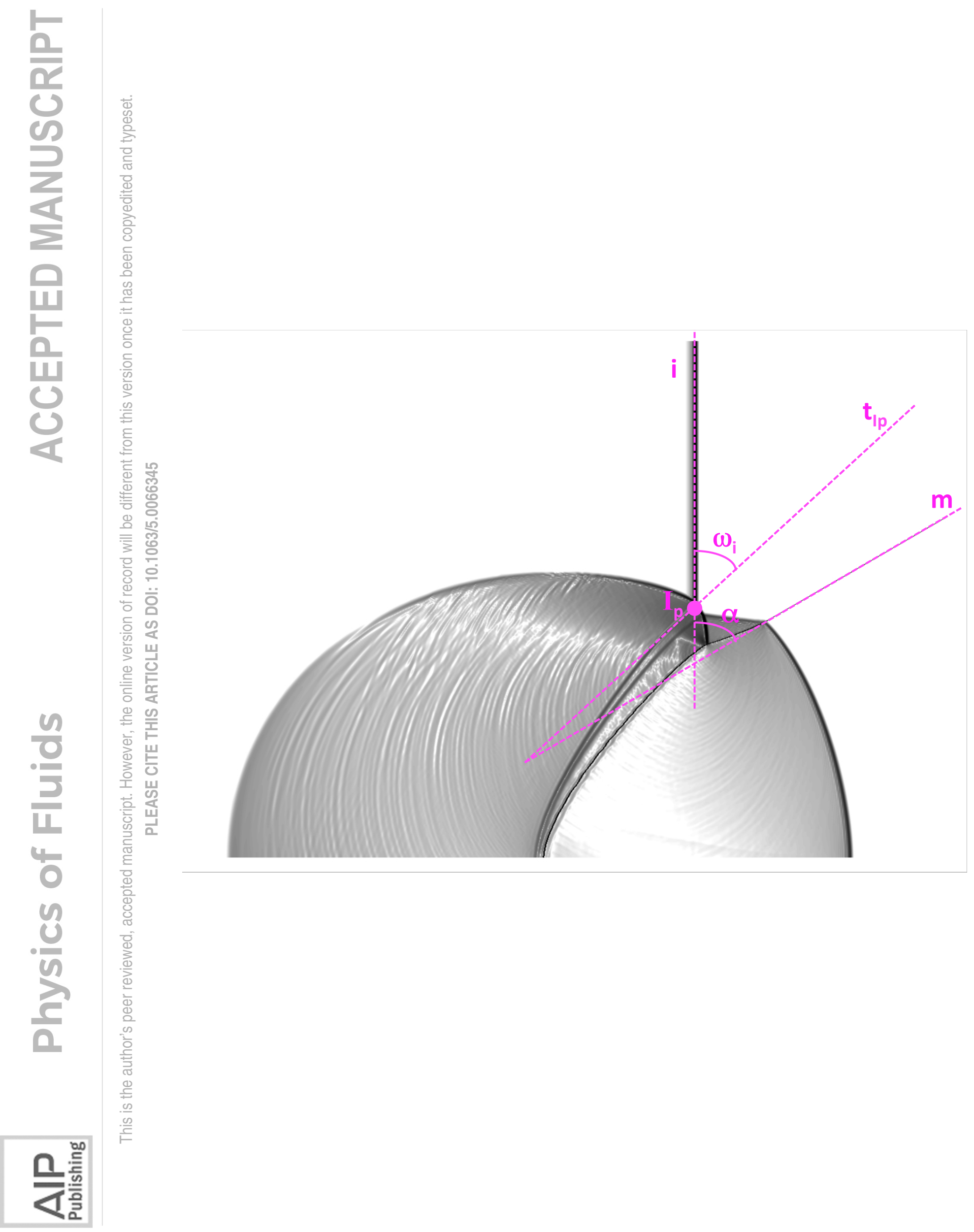




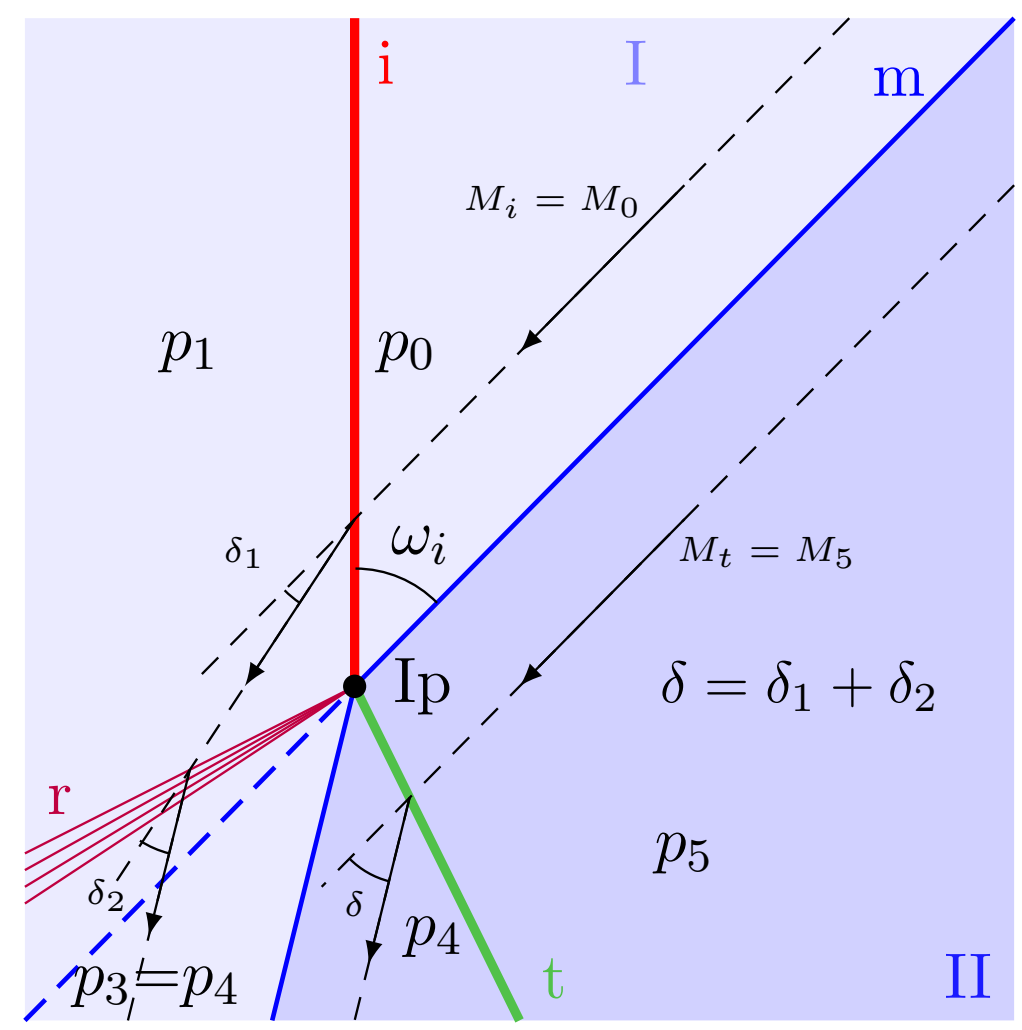

Figure 1: Flow description for a Regular Refraction with a reflected Expansion (RRE) in the frame of reference attached to the incident point Ip. 


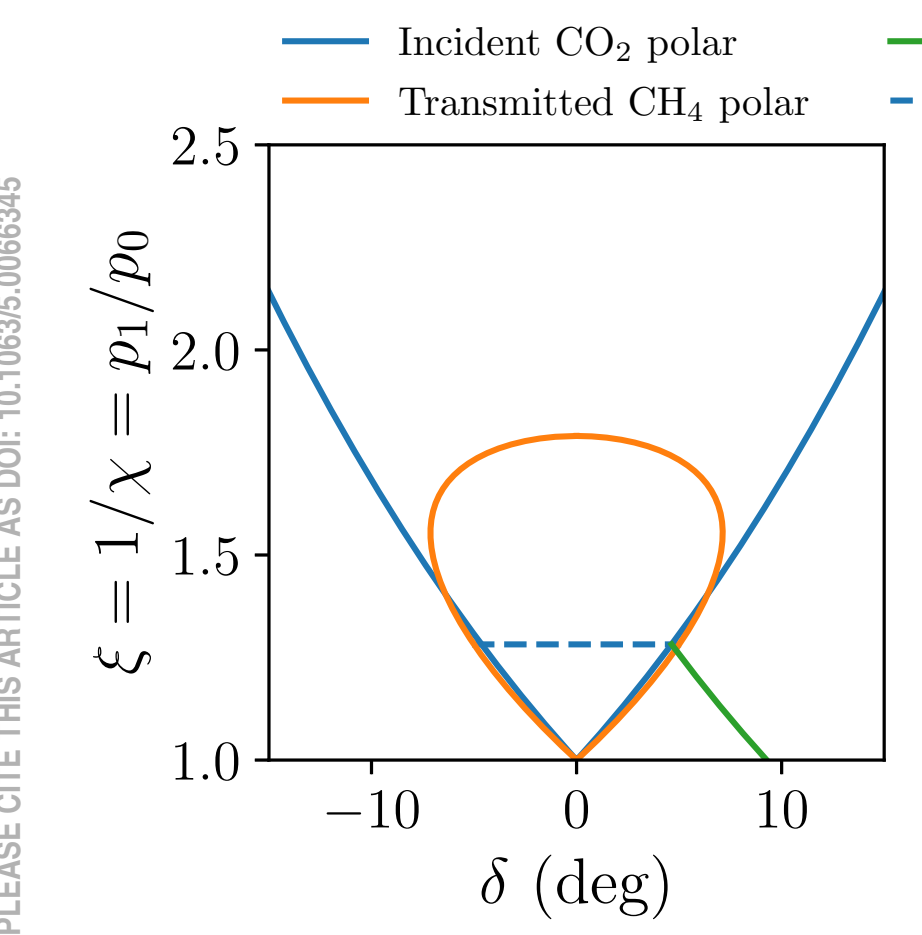

Reflected expansion

- - - Incident pressure ratio, $\xi$

$n$
$\frac{0}{3}$
$\frac{3}{4}$
4
0
0
$\frac{0}{n}$
$\frac{1}{2}$

(a) $M_{i}=2.1712 ; \quad \omega_{i}=31^{\circ}$

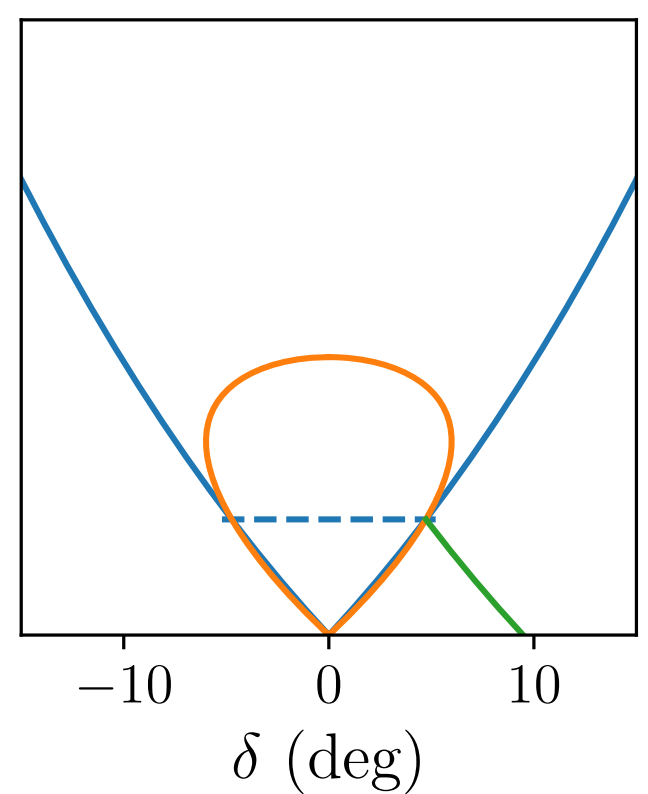

(b) $M_{i}=2.1067 ; \quad \omega_{i}=32.06^{\circ}$ 


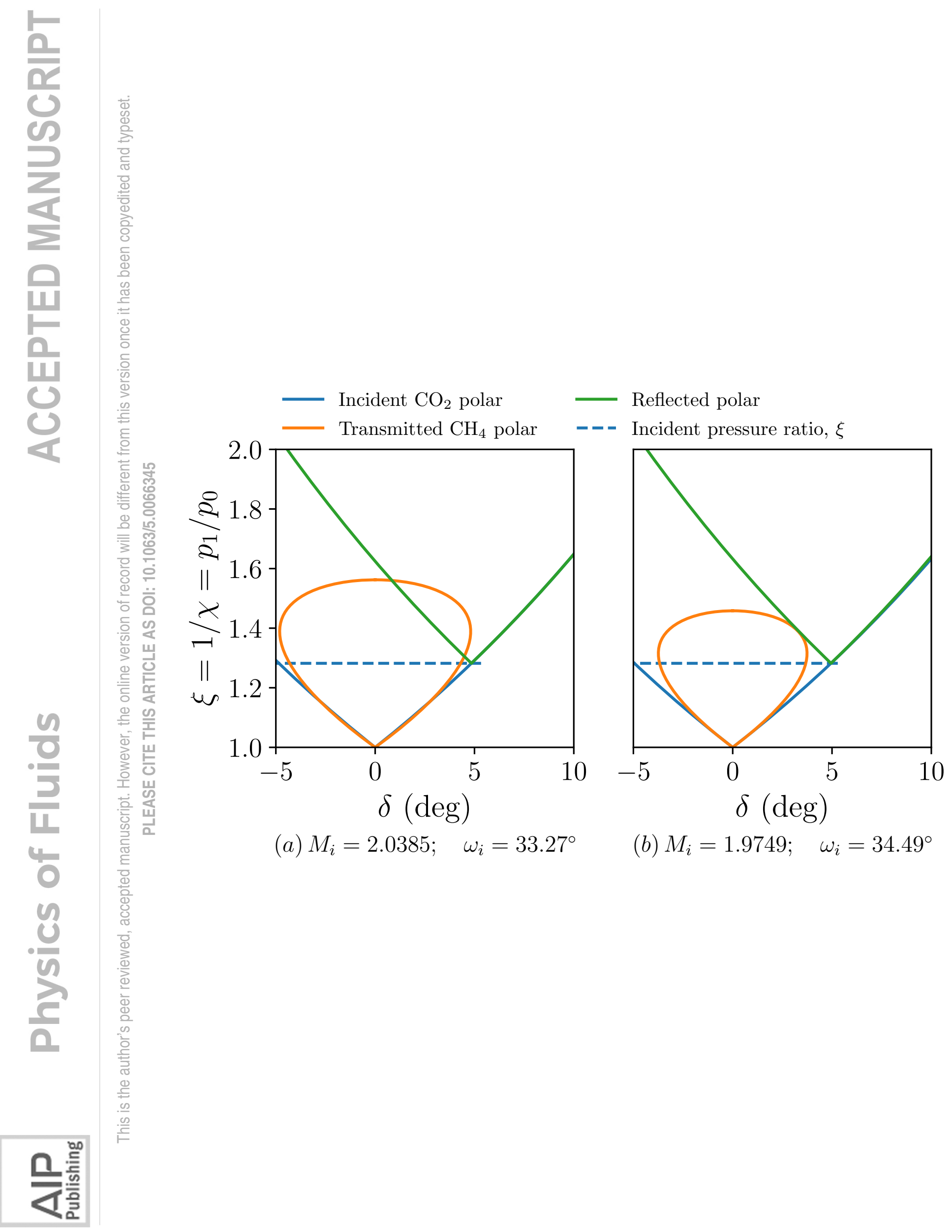




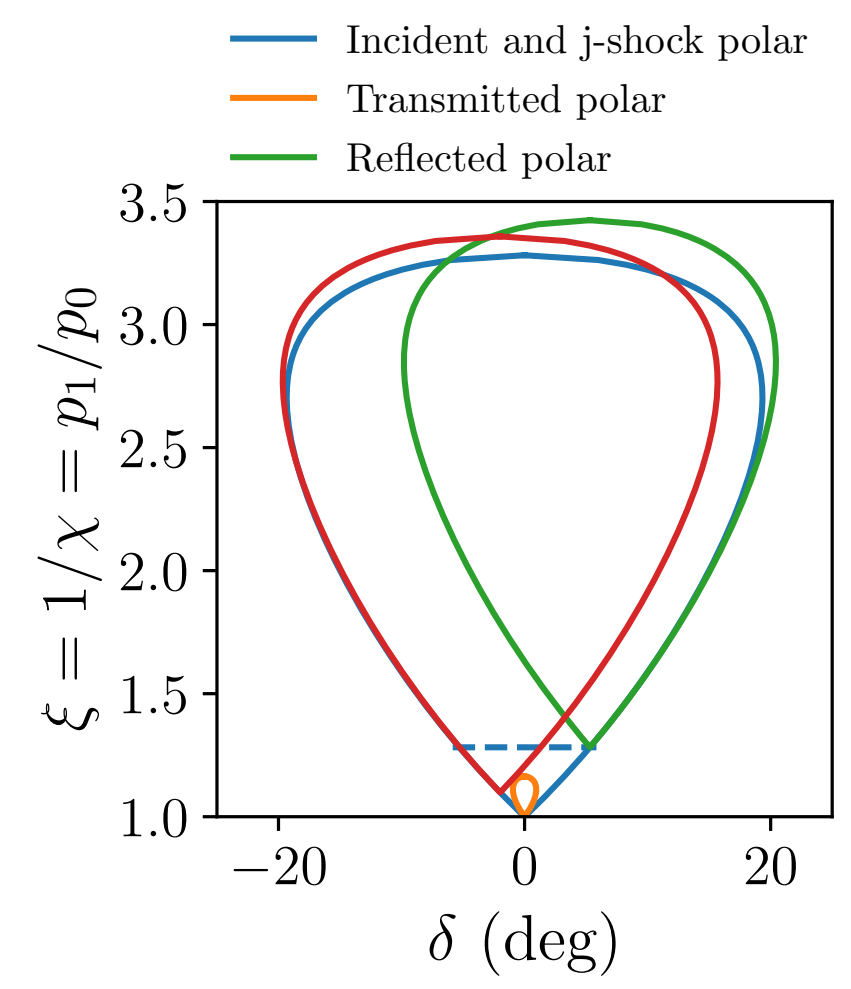

(a) $M_{i}=1.7397 ; \quad \omega_{i}=40^{\circ}$
_ k-shock polar

- - - Incident pressure ratio, $\xi$

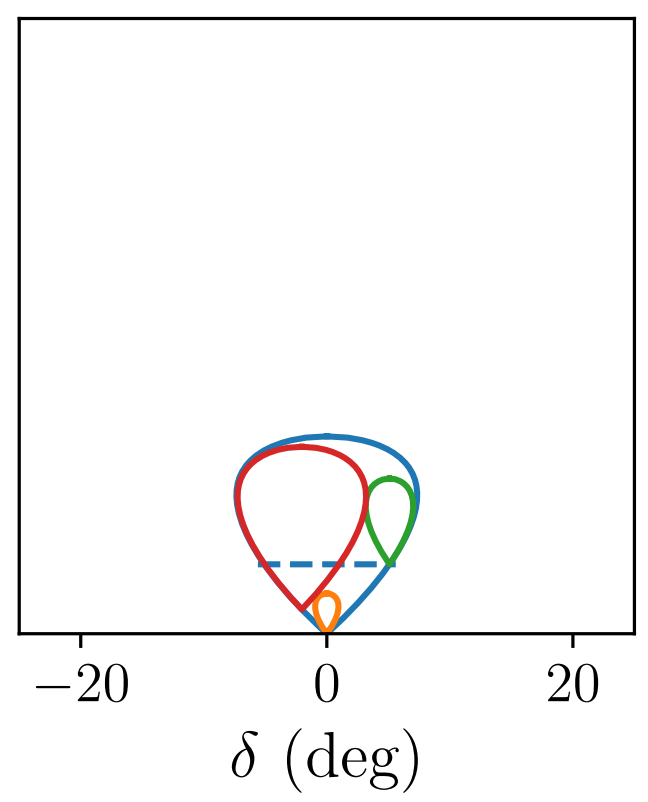

(b) $M_{i}=1.3086 ; \quad \omega_{i}=58.71^{\circ}$ 
- - Theoretical limits

O Abd-el-Fattah \& Henderson (1978)

ceceso Present CFD: RRE; RRR; BPR; FPR; TNR; LSR

Nourgaliev et al. (2005): TRR $\rightarrow$ TNR;

$\square \mathrm{RRE} \rightarrow$ Intromission $\rightarrow \mathrm{RRR} \rightarrow$ Shock critical $\rightarrow \mathrm{BPR} \rightarrow$ FPR; $\mathrm{RRE} \rightarrow \mathrm{BPR} \rightarrow \mathrm{TMR}$

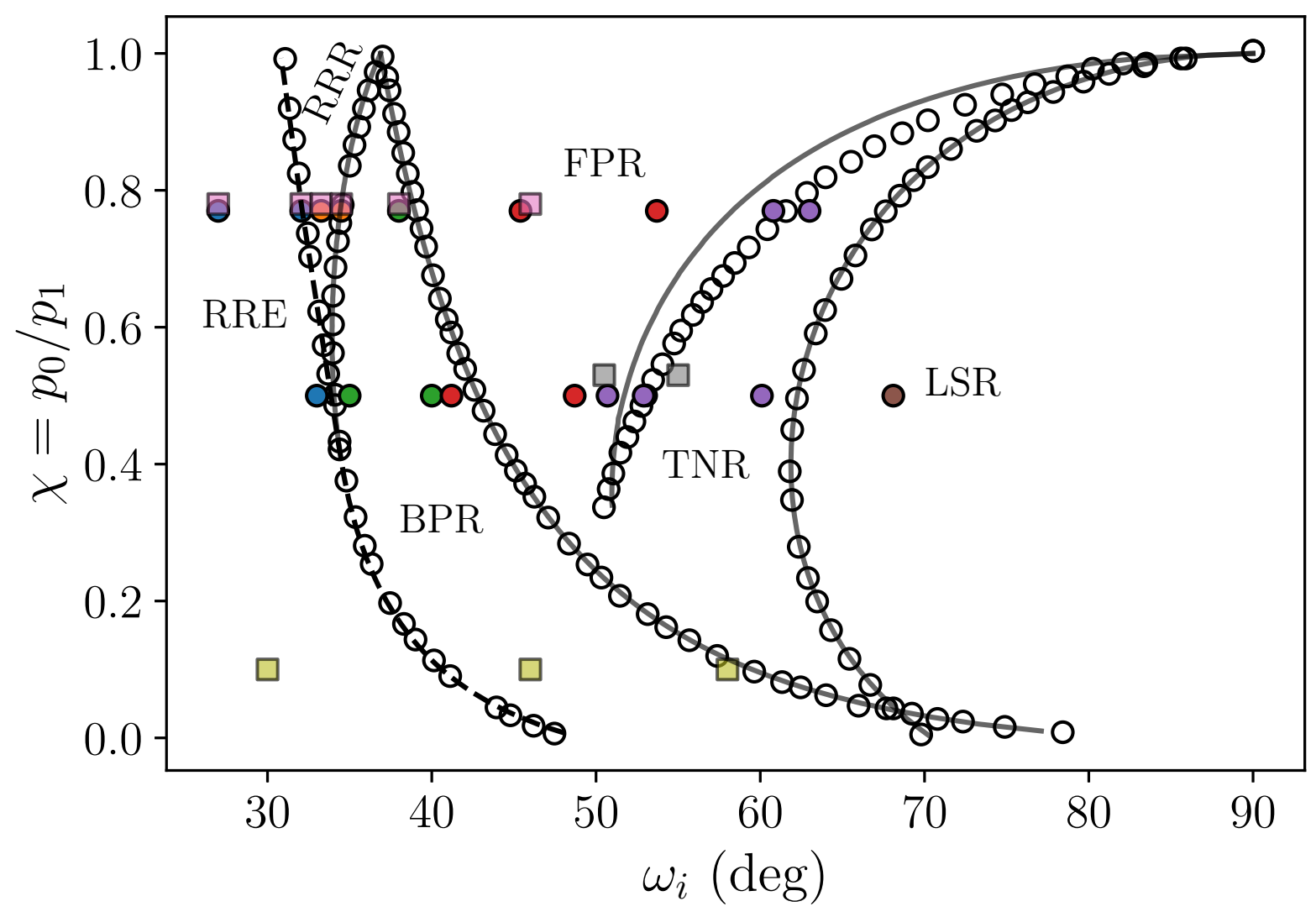




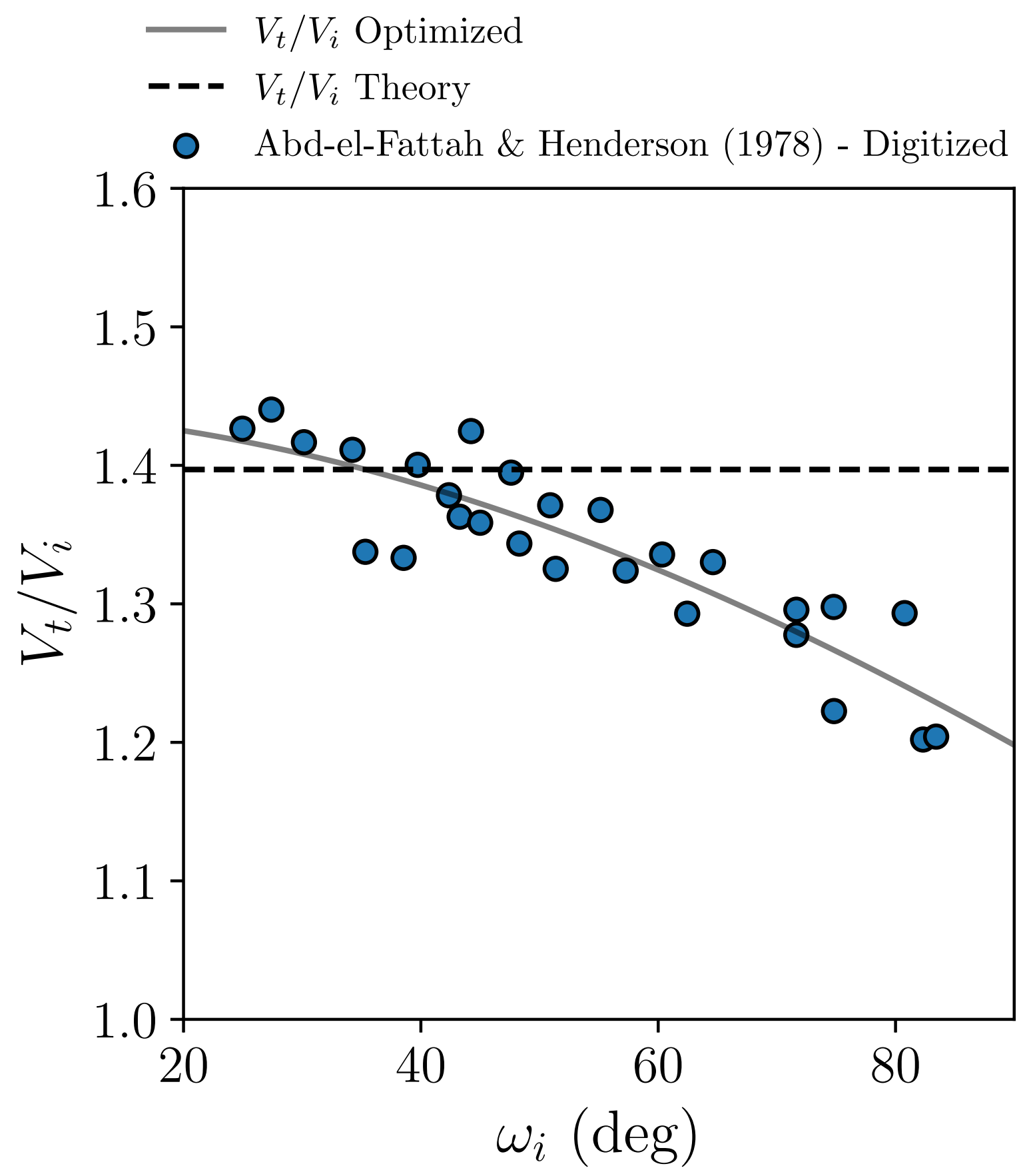




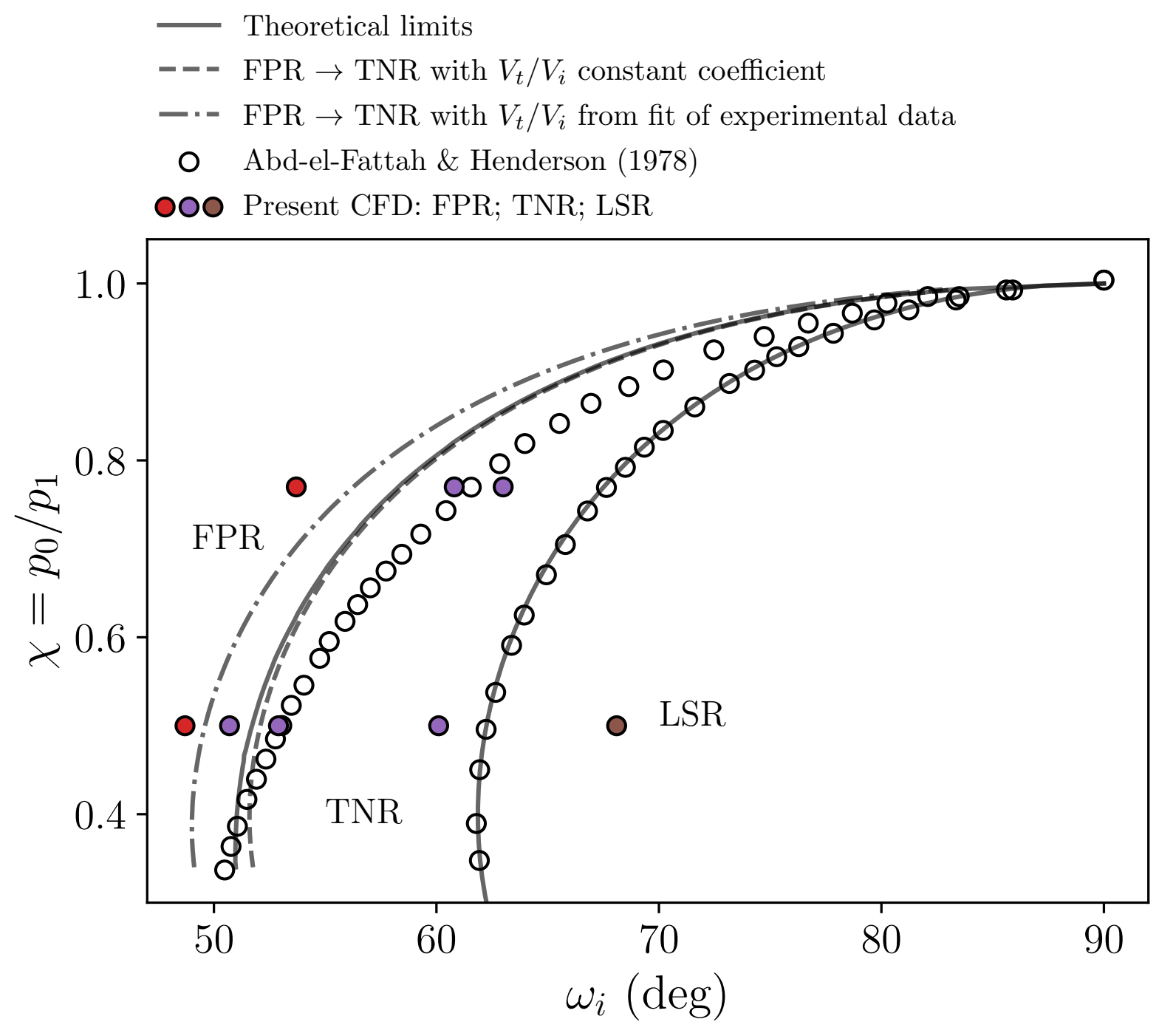



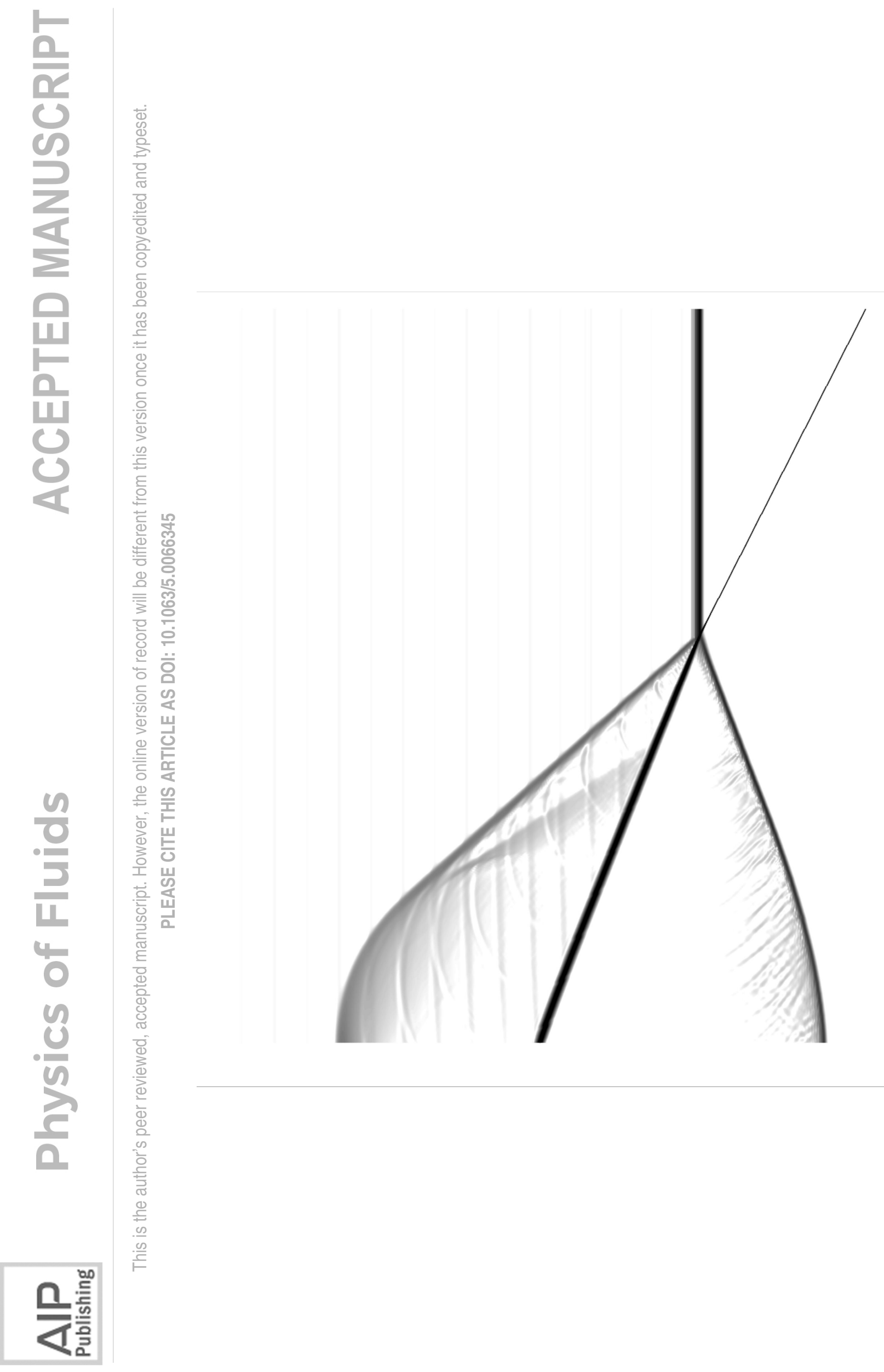


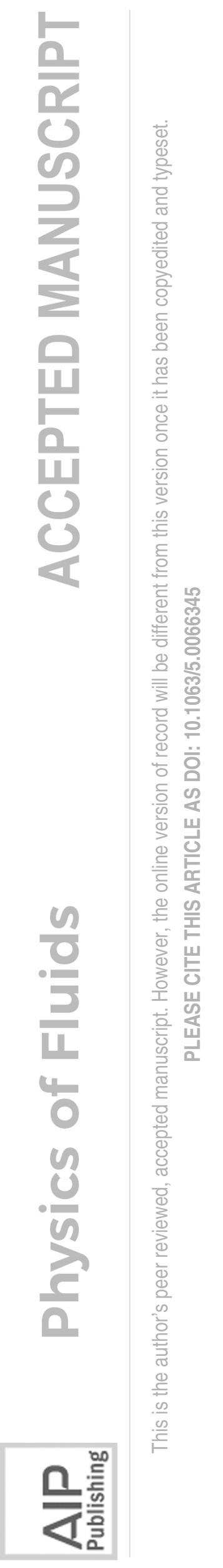



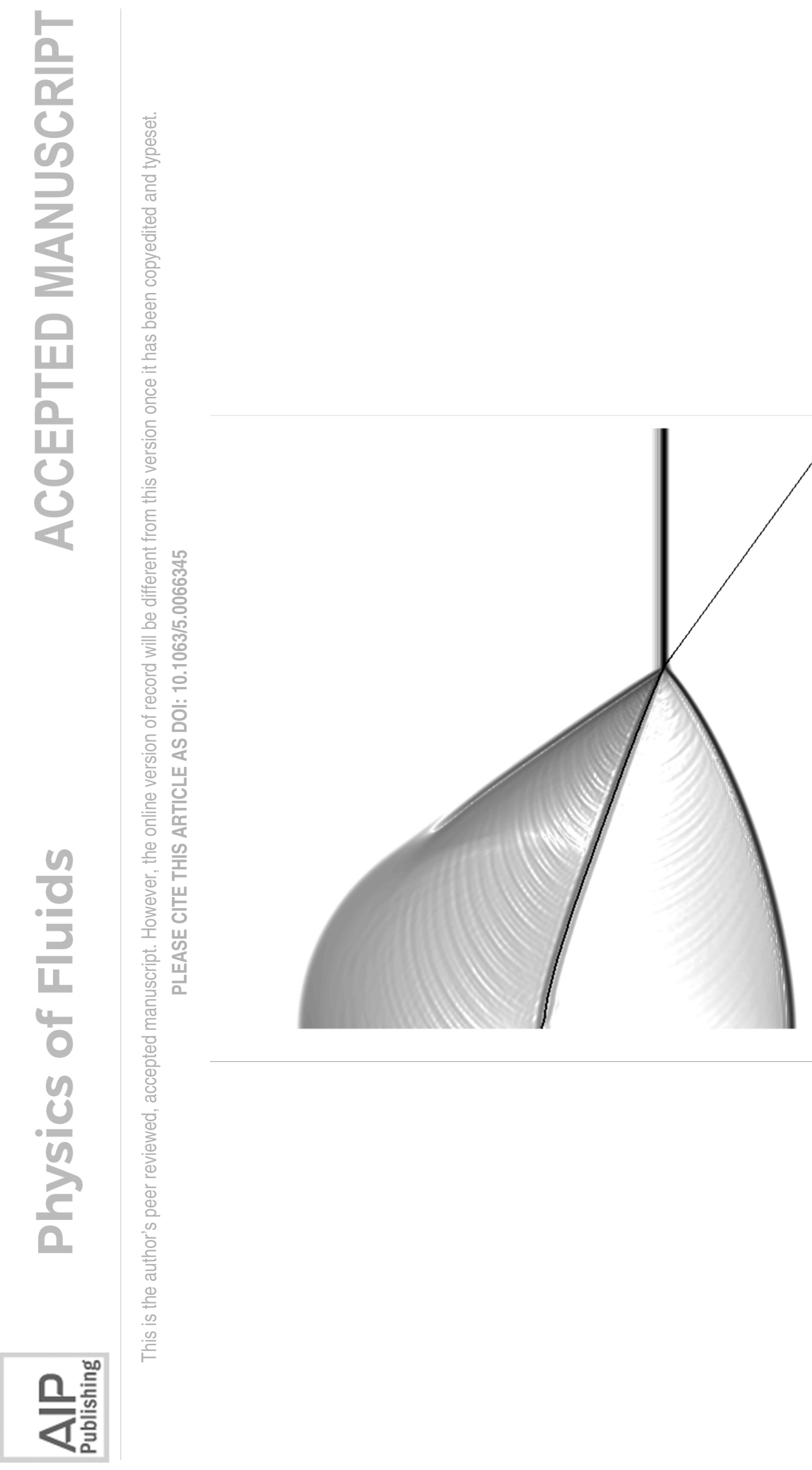


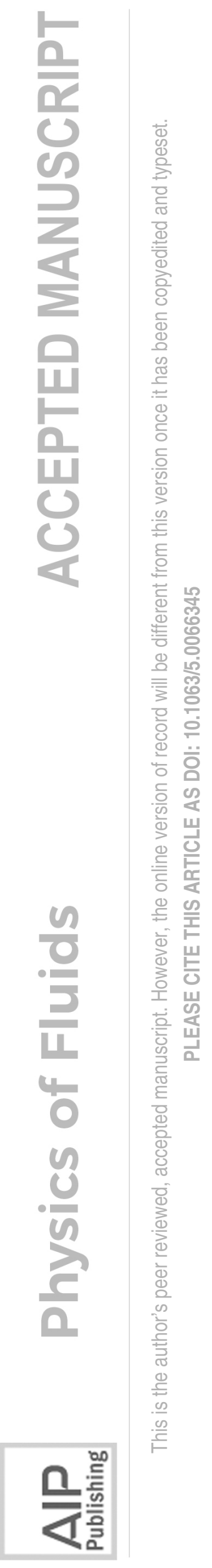




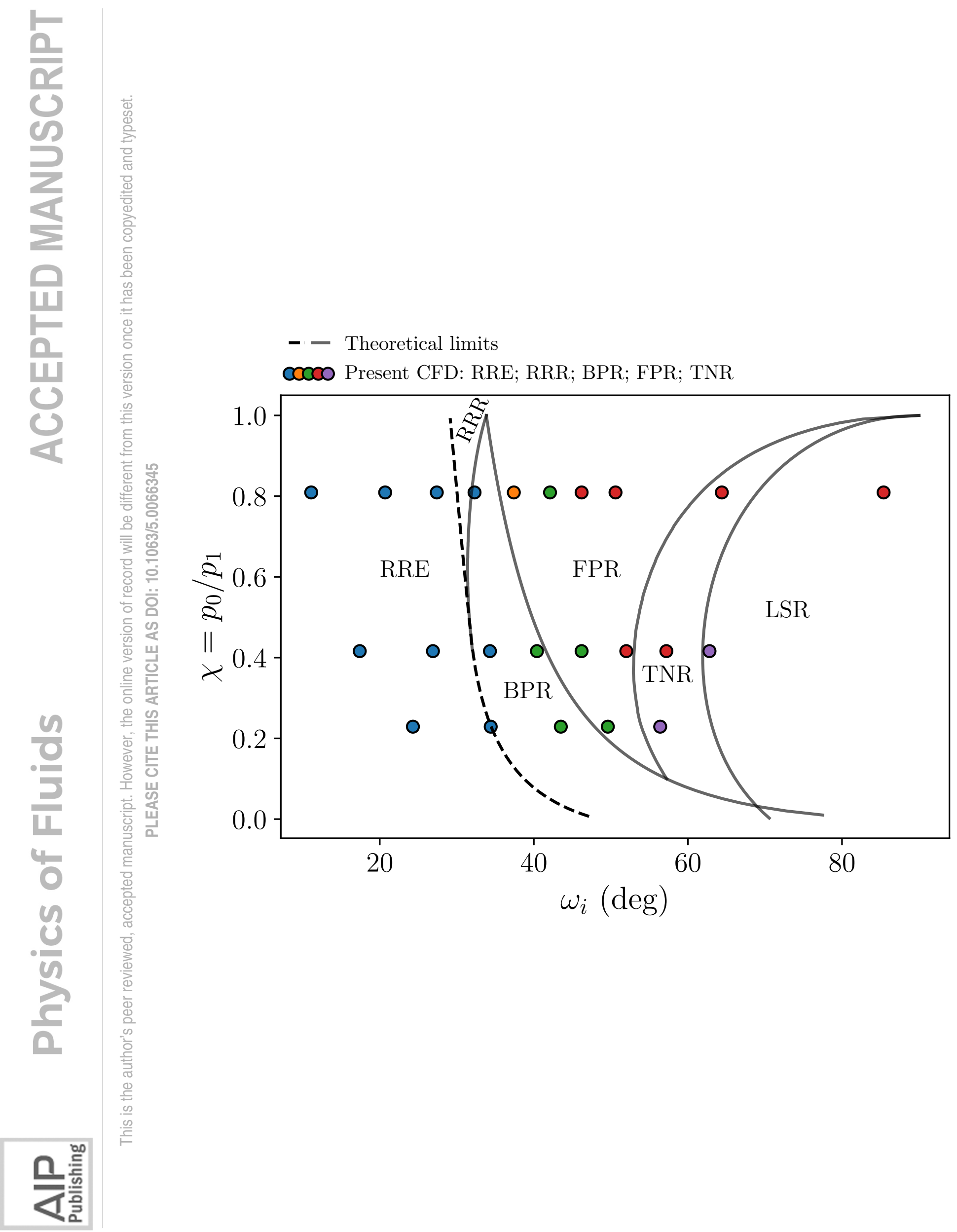



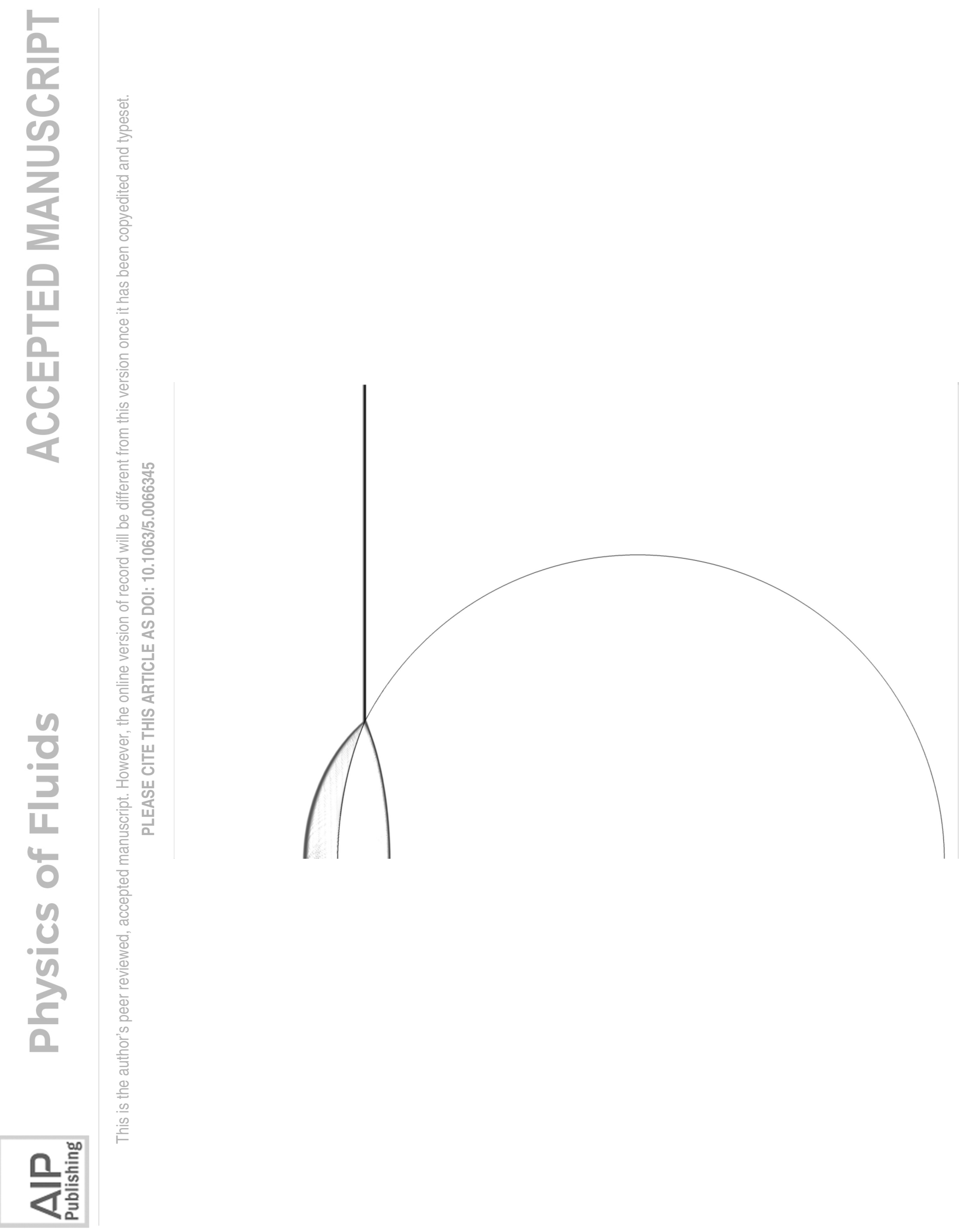



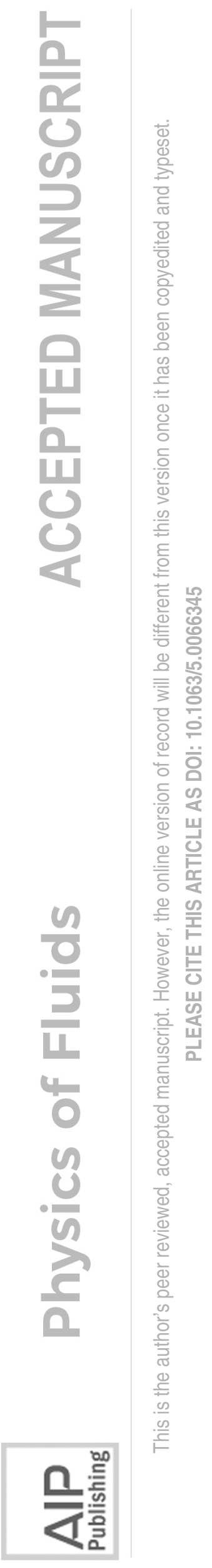



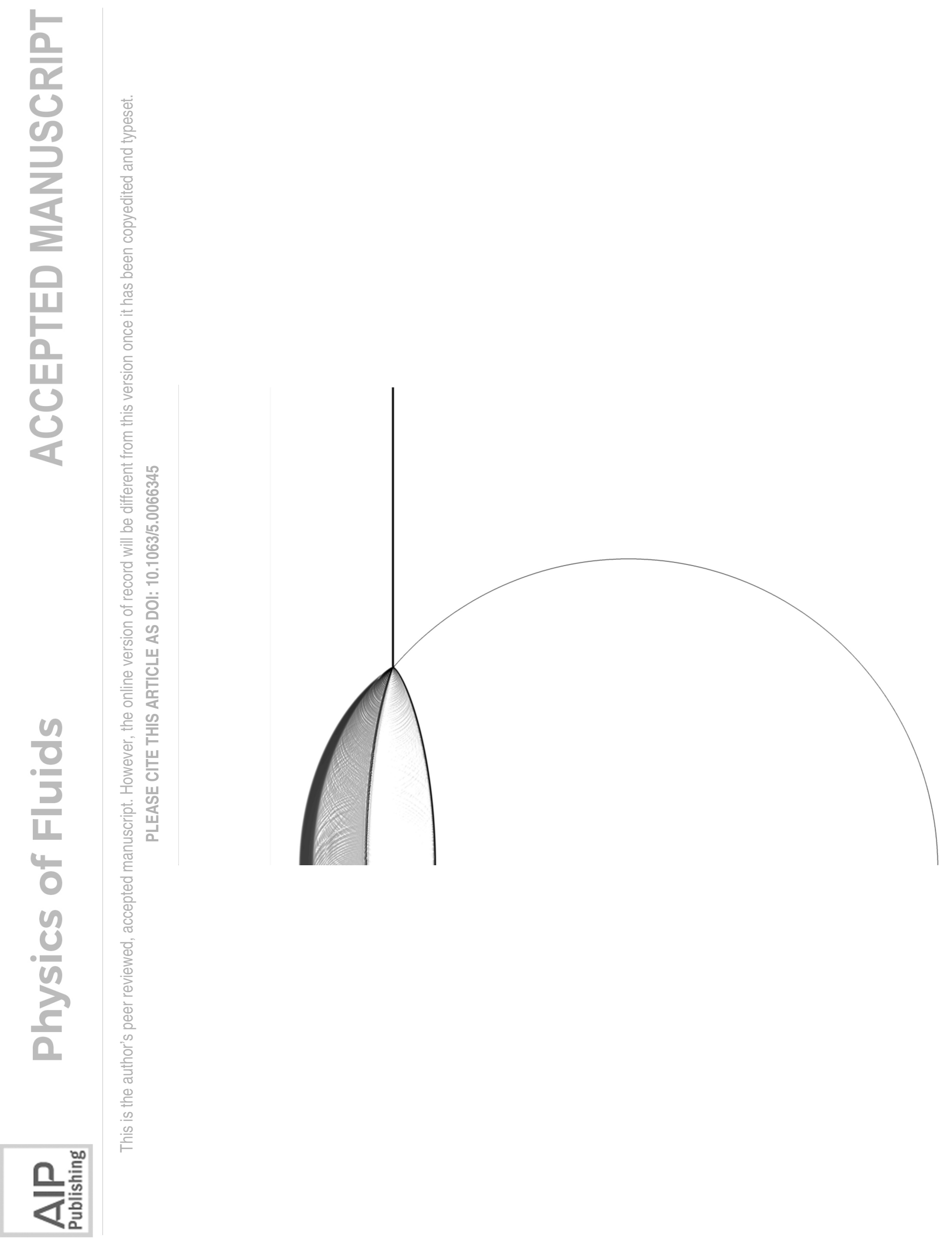

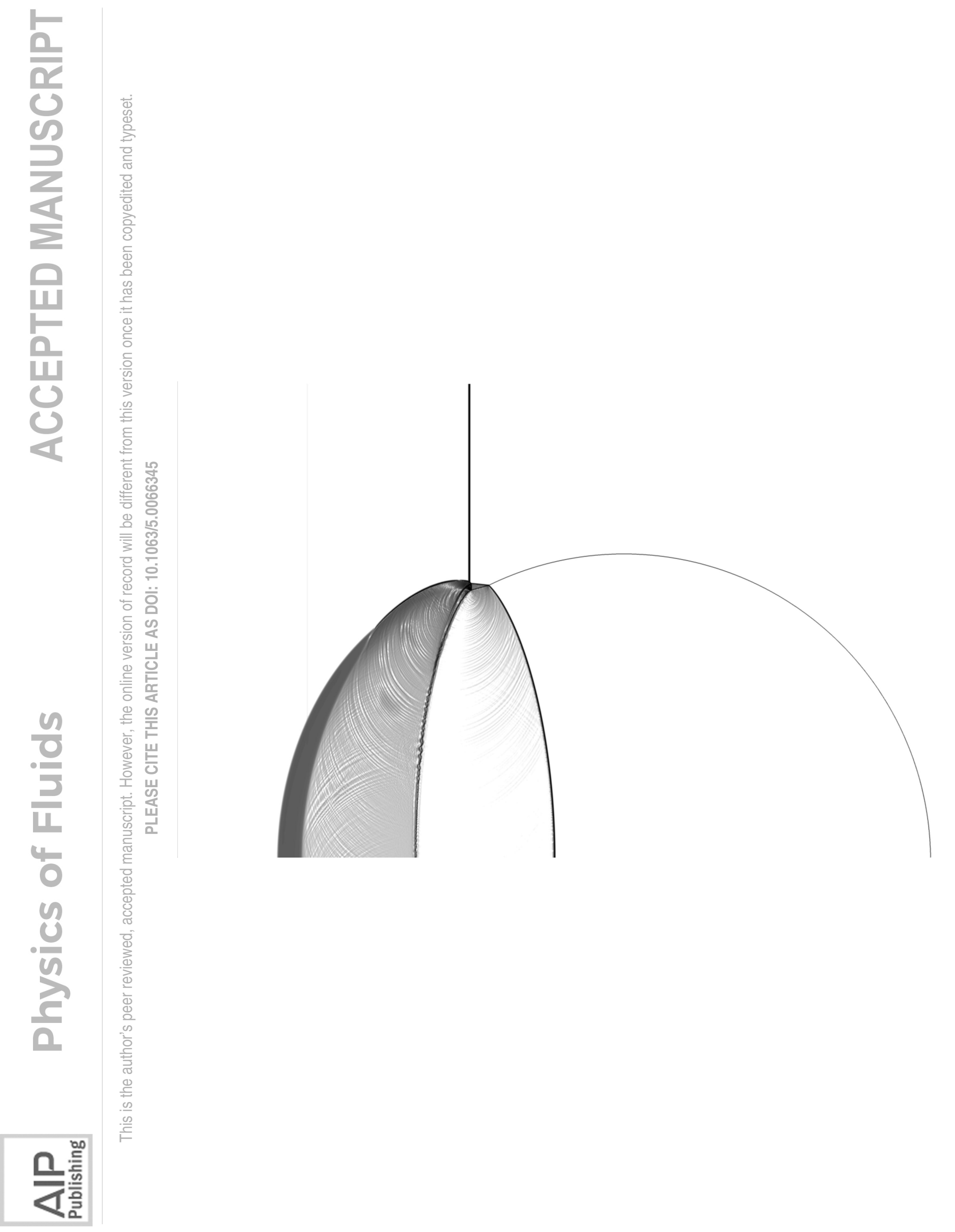

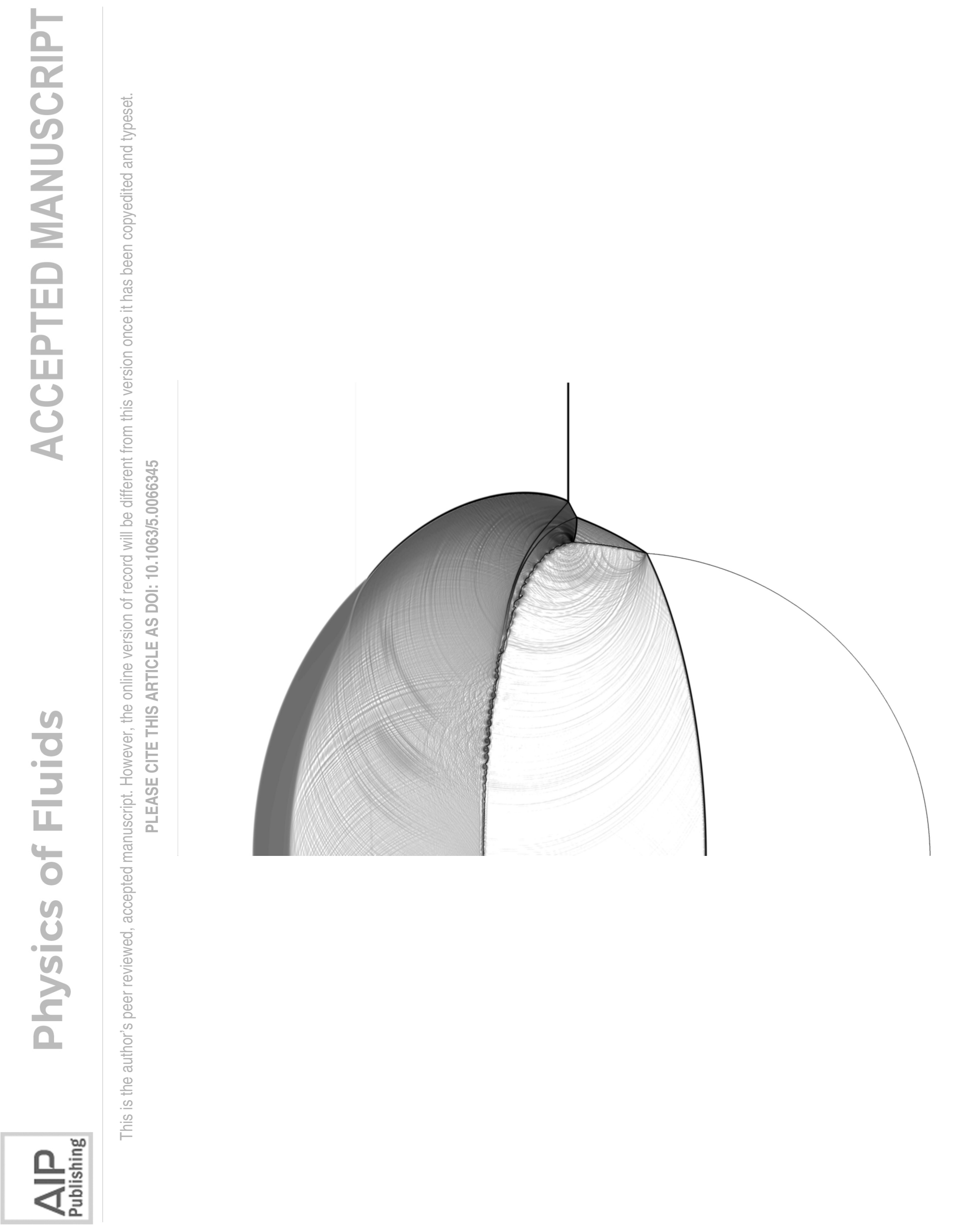

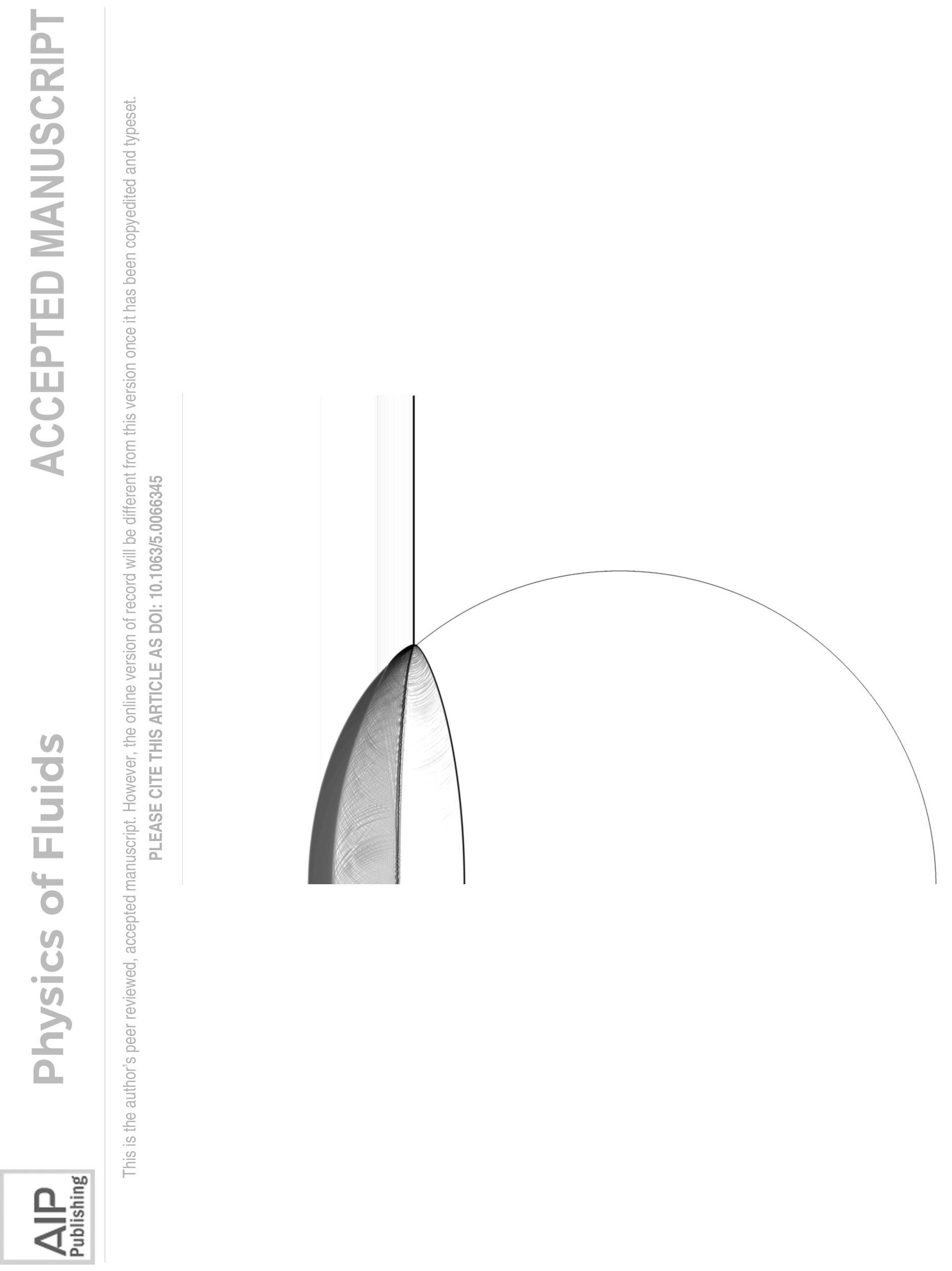

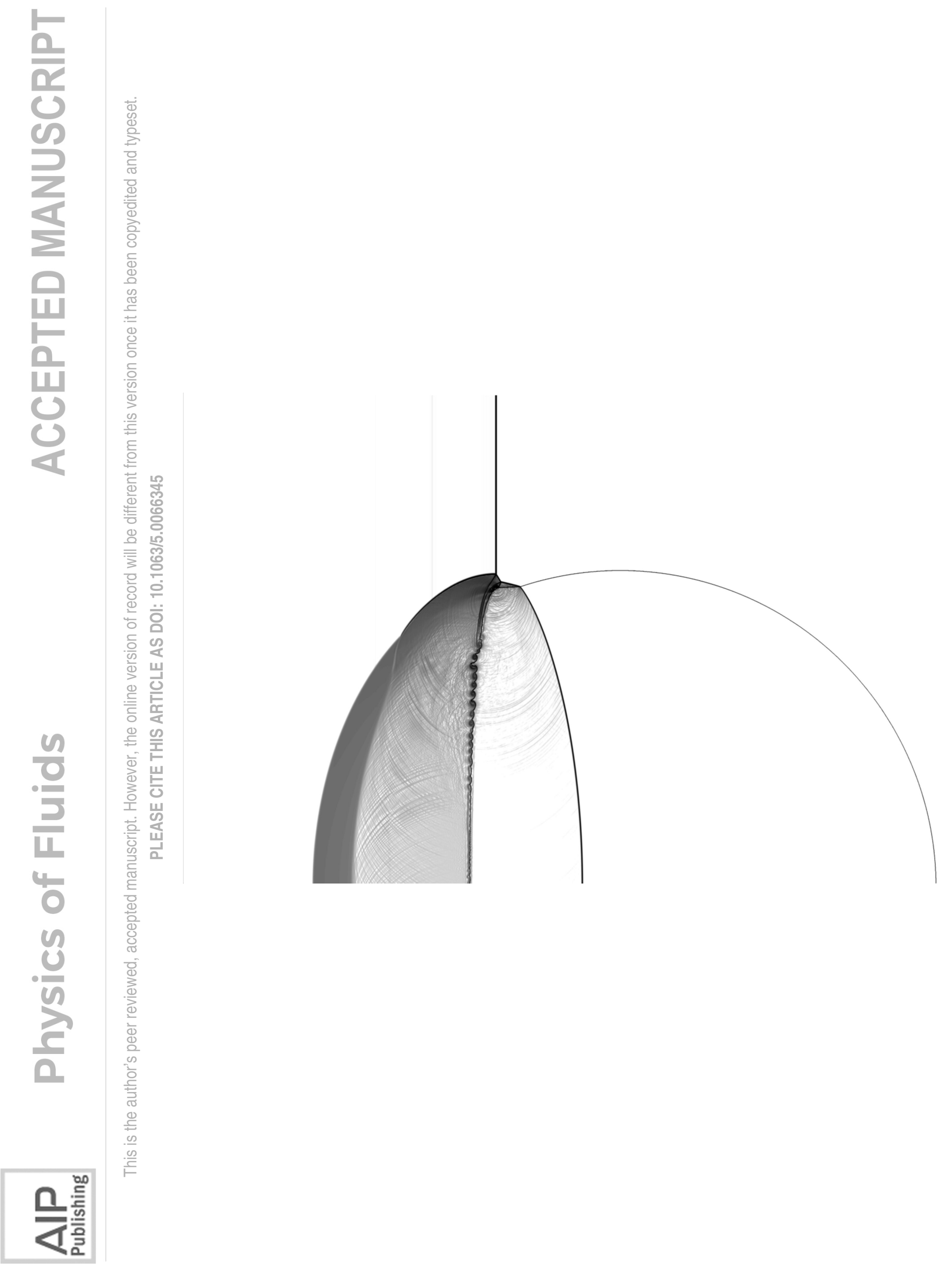

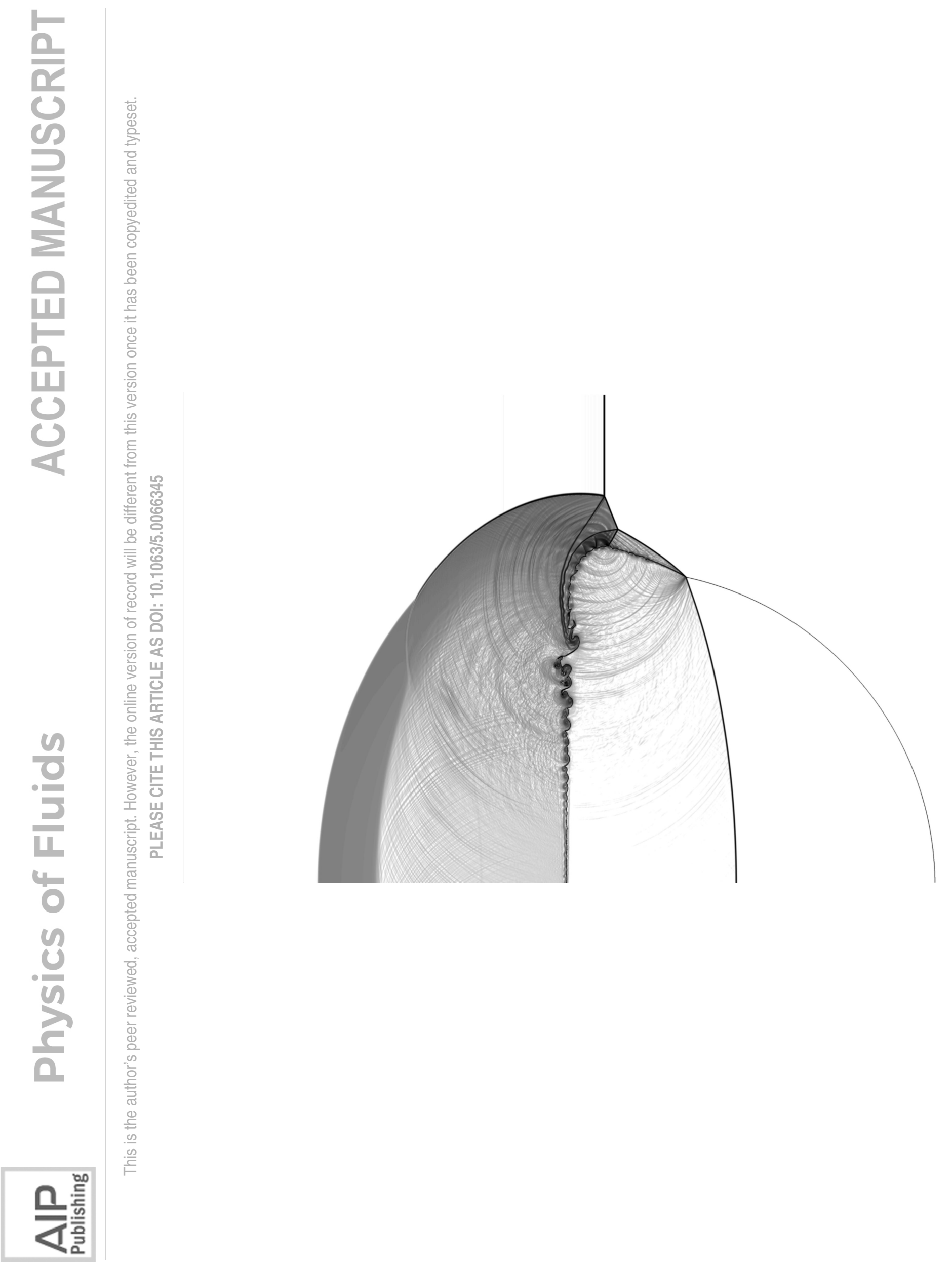


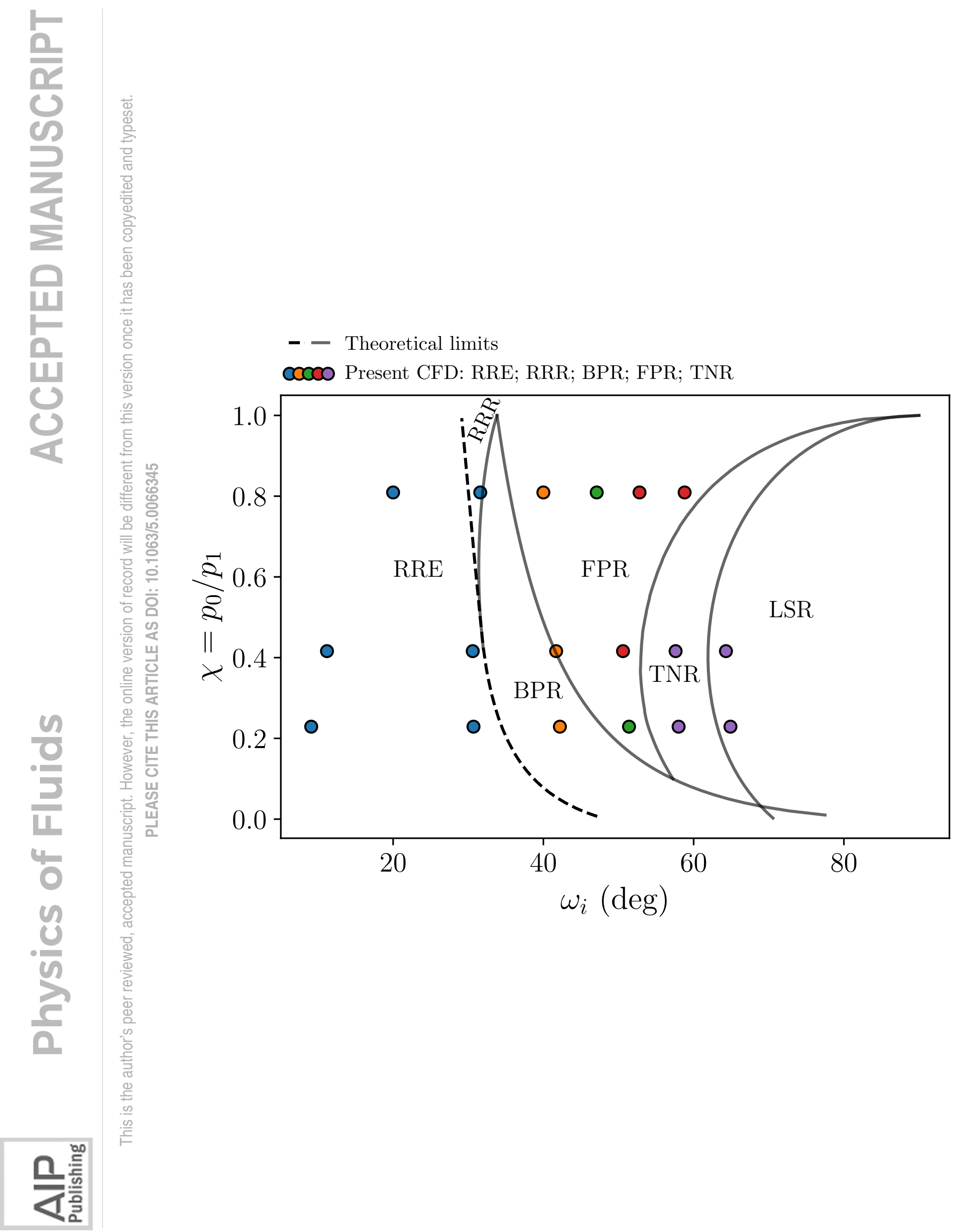




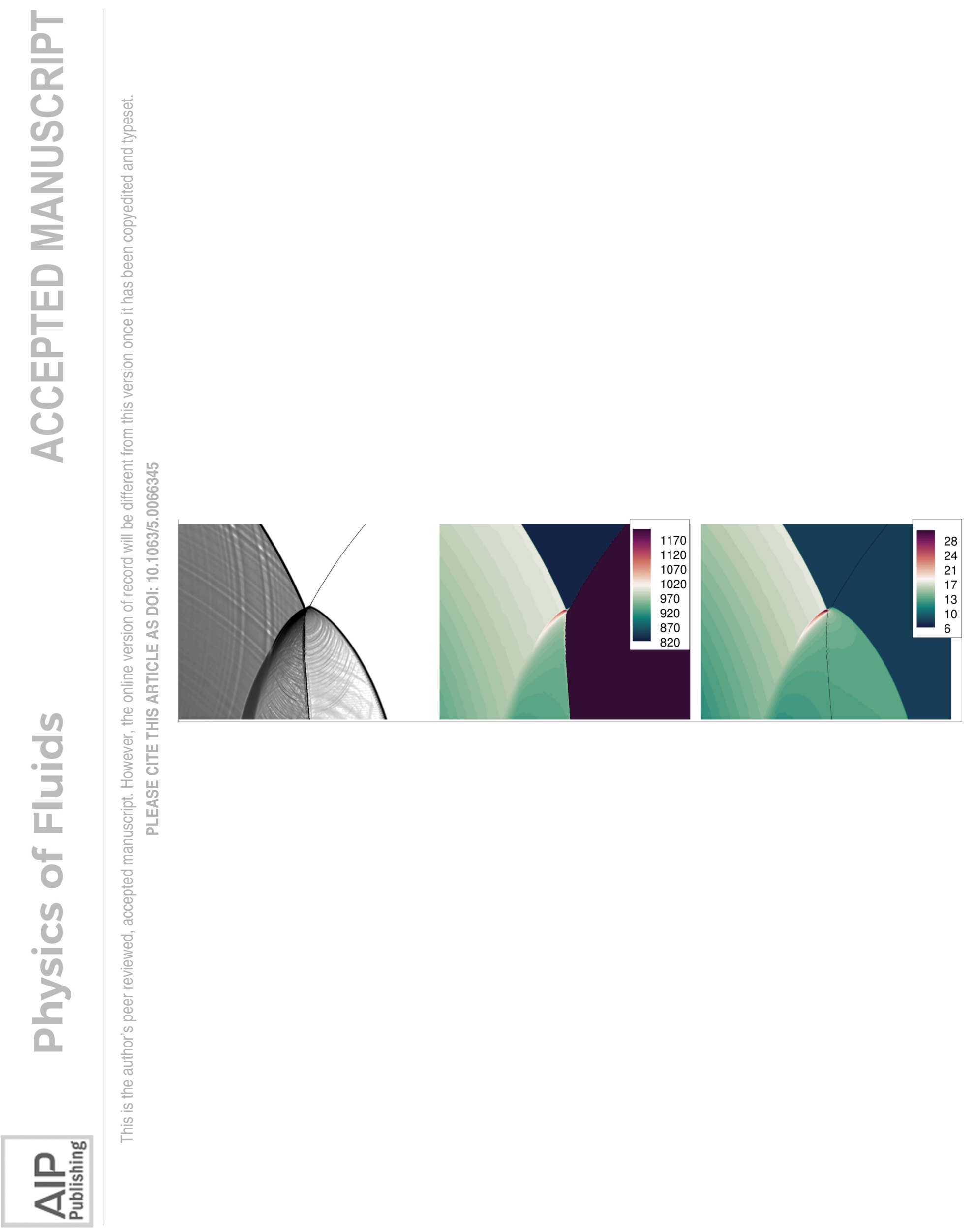




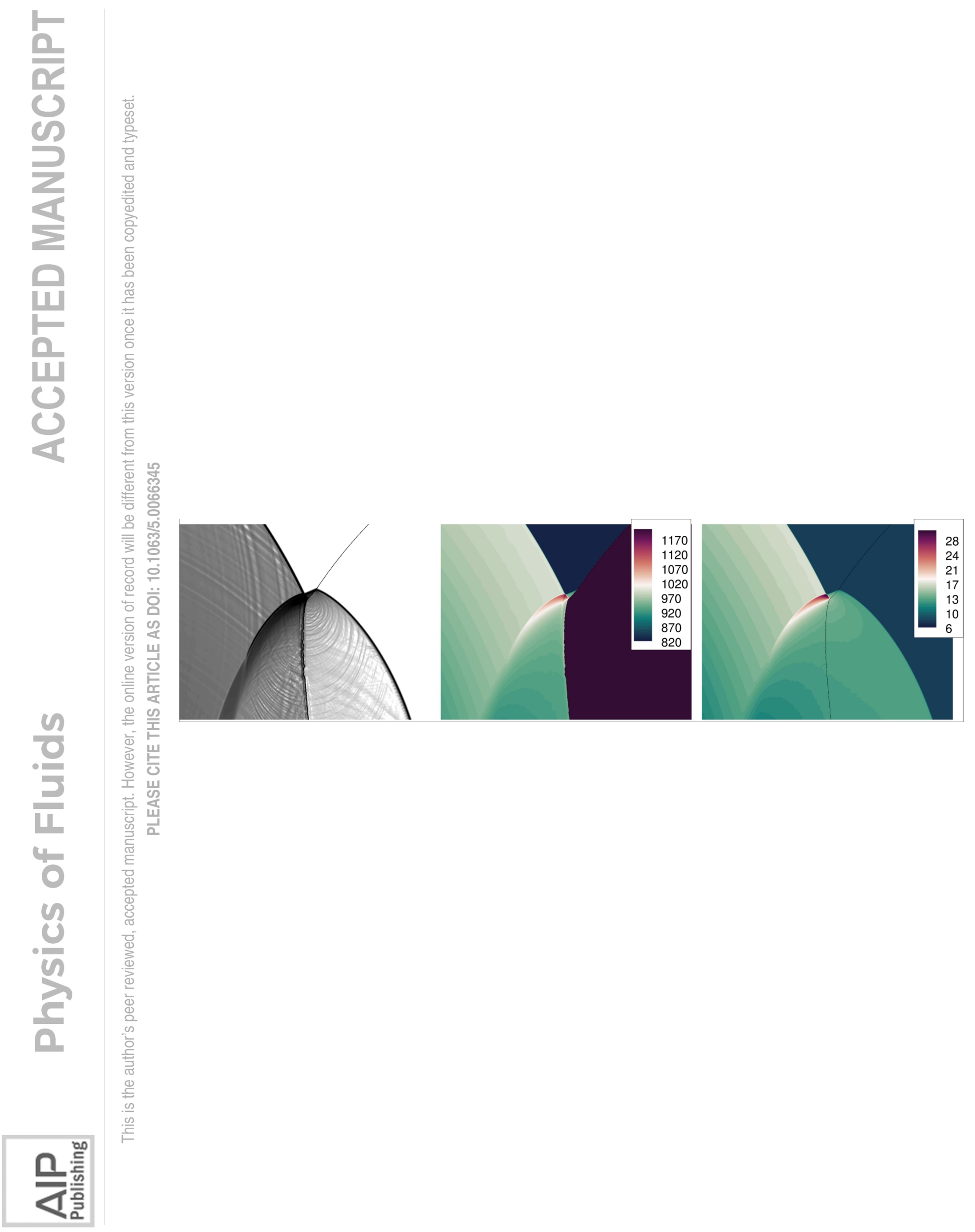




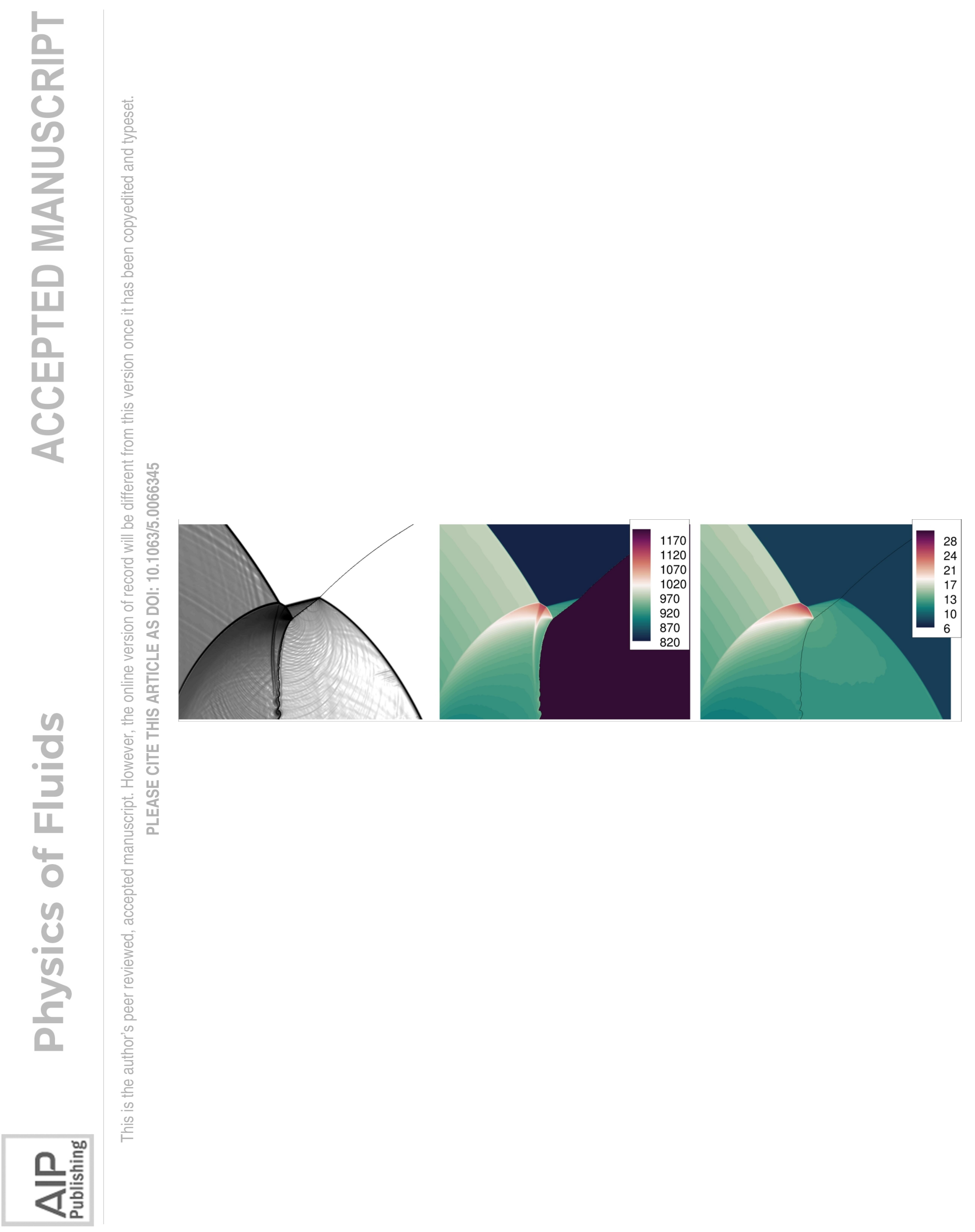




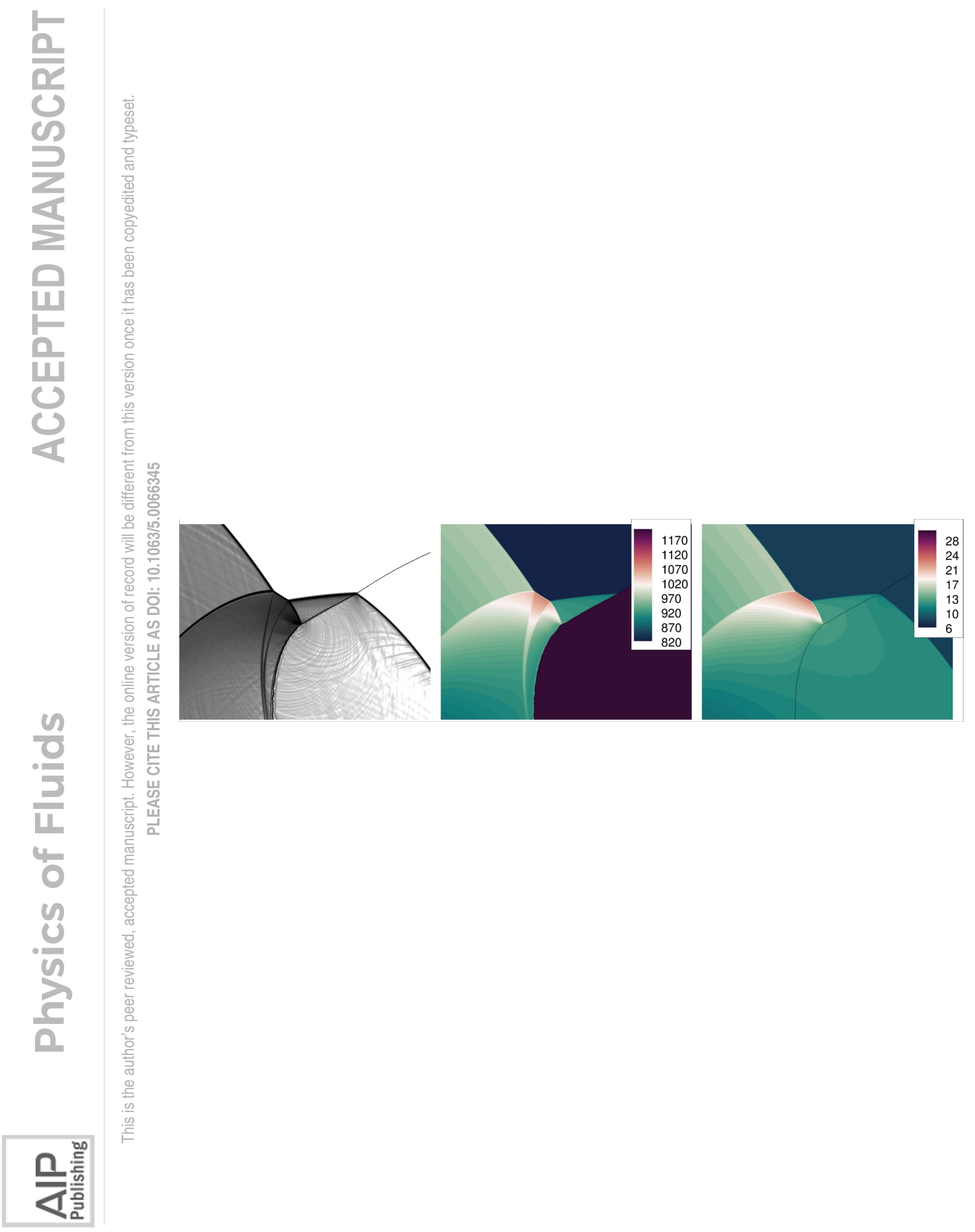


$\square \square \square$ Planar shock: $M=1.1 ; 1.5 ; 2.0$

o०० Curved shock: $M=1.1 ; 1.5 ; 2.0$
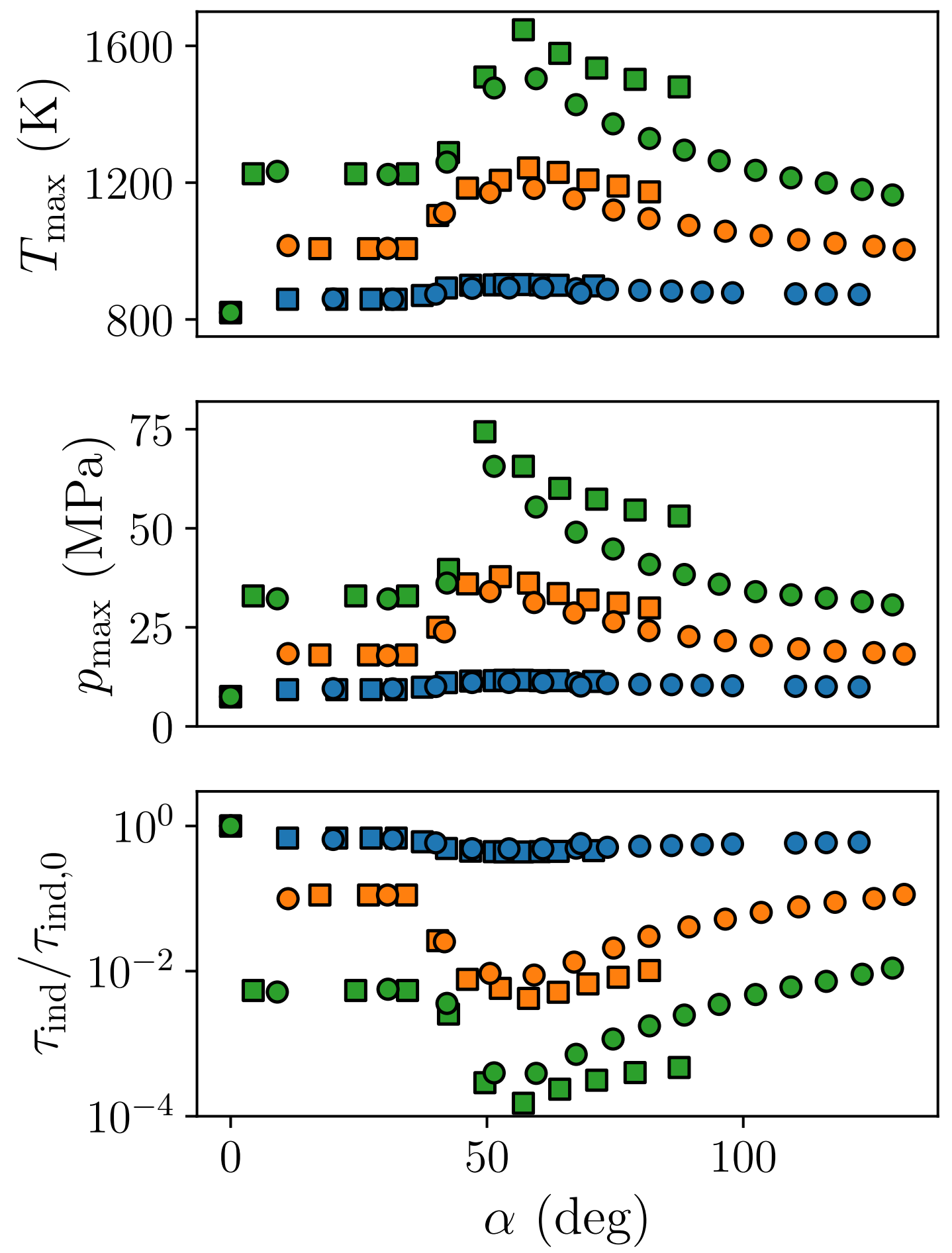